\title{
Carbonatites and associated nephelinites from São Vicente, Cape Verde Islands
}

\author{
C. De Ignacio*, M. Muñoz And J. Sagredo
}

Departamento Petrología y Geoquímica, Facultad de Ciencias Geológicas, Universidad Complutense de Madrid, C/ José Antonio Novais, 2, 28040 Madrid, Spain

[Received 17 January 2011; Accepted 15 June 2011; Associate Editor: Attila Demény]

\section{ABSTRACT}

The island of São Vicente has the most abundant carbonatite outcrops in the Cape Verde Islands. A field survey of the main outcrops has shown that they consist of extrusive carbonatites, carbonatite dykes and small apophyses of intrusive carbonatite. These outcrops are spatially related to nephelinites. The compositions of the extrusive carbonatites and dykes plot close to, and within, the magnesiocarbonatite field. In contrast, the intrusive carbonatites are calciocarbonatites, with similar average strontium contents to those of extrusive carbonatites and dykes (around $4000 \mathrm{ppm}$ ), but remarkably low barium, niobium and total rare earth element concentrations. Whole-rock geochemistry indicates a strong affinity between the nephelinites and intrusive carbonatites, such that the latter could represent fractionation products of the same parental magma. This is in agreement with radiogenic isotope geochemistry, which shows a very restricted range of compositions in the $\mathrm{Sr}, \mathrm{Nd}$ and $\mathrm{Pb}$ systems. Fractionation from a common parental magma occurred in two main steps: high-temperature nephelinite crystallization and high-temperature carbonatite immiscibility. The carbonatitic melts crystallized in two different environments, as follows: (1) a shallow intrusive environment, giving rise to the early calciocarbonatite cumulates; and (2) a vapour-dominated, extrusive environment, producing the later magnesiocarbonatites.

KEYwORDs: carbonatites, extrusive, intrusive, nephelinites, Cape Verde Islands.

\section{Introduction}

CARbonatites have been reported from two islands in the northern part of the Cape Verde archipelago, São Vicente (Gerlach et al., 1988) and São Nicolau (Kogarko, 1993). São Vicente has the greatest number of carbonatite exposures (Gerlach et al., 1988), including calcitic and dolomitic types. The carbonatites are associated with alkaline silicate rocks, and resemble the alkaline-carbonatitic association found on Fuerteventura (the only island in the Canary Islands where carbonatites crop out). The study of the São Vicente carbonatites and alkaline silicate rocks provide an opportunity to expand our

* E-mail: cris@geo.ucm.es

DOI: $10.1180 /$ minmag.2012.076.2.05 understanding of this kind of magmatism in an oceanic context. São Vicente's alkaline and carbonatitic magmatism is much younger than that on Fuerteventura. It allows connections between eruptive material and the roots of the volcanic system to be established, thus contextualizing the whole magmatic system.

There are several works dealing with the origin of the Cape Verde archipelago (e.g. Holm et al. 2008), and many focusing on the mantle sources of alkaline and carbonatitic magmas (Gerlach et al., 1988; Hoernle et al., 2002; Doucelance et al., 2003; 2010; Mata et al., 2010). Relatively little has been published on the field relations, textures, mineral chemistry and whole-rock geochemistry of the São Vicente carbonatites. These are key parameters in the construction of petrogenetic models for these rocks. In the description of 
carbonatites, an aspect of particular interest is their possible relation to alkaline silicate rocks. On São Vicente, nephelinites are closely related to carbonatites in space and time; they must also be studied to fully understand carbonatite genesis.

In this work we present field, petrographic, mineralogical and geochemical data on the São Vicente carbonatites and nephelinites. The petrography and mineral chemistry are important. In many alkaline-carbonatitic complexes it is difficult to identify the parental compositions of the carbonatites and associated alkaline silicate rocks. It is only by the combined study of petrography and mineral chemistry that information about possible accumulation processes or different magmatic interactions (such as the occurrence of xenocrysts or reactions) that affect whole-rock geochemistry is revealed. In turn, the integration of textural, mineralogical and geochemical data with field relations and isotope geochemistry provides valuable information about the processes that give rise to this kind of magmatism.

\section{Geological setting}

The Cape Verde volcanic archipelago is located on the oceanic portion of the African plate, $\sim 500 \mathrm{~km}$ west of Senegal. There are ten major islands and a number of smaller ones, arranged in a westward-facing horseshoe. They are divided into the northern Windward Isles and the southern Leeward Isles (Fig. 1).

The dating of the volcanic and intrusive units on several islands (e.g. Mitchell et al., 1983; Plesner et al., 2002; Huertas et al., 2006; Holm et al., 2008; Ancochea et al., 2010) suggests that most of the volcanic activity took place from $16 \mathrm{Ma}$ to the present. The activity on Fogo may be entirely Quaternary in date, on Maio it is relatively old (12-7 Ma), on Santo Antão and São Vicente the range is from 7.5 to $0.1 \mathrm{Ma}$, and 9.0 to $0.3 \mathrm{Ma}$, respectively.

Most of the Neogene volcanic rocks are olivine-nephelinites and basanites, together with a few phonolitic plugs, tephrite dykes and nepheline-syenite plutons. Carbonatites have been described in a number of publications including Assunção et al. (1965), Machado et al. (1968), Alves et al. (1979), Silva et al. (1981) and Kogarko et al. (1993); they crop out on at least in six of the islands (Maio, Fogo, São Tiago, Brava and surrounding islets in the south, and São Nicolau and São Vicente in the north of the archipelago). Recent descriptions include Gerlach et al. (1988), Hoernle et al. (2002) and Mourão et al. (2010).

Three concentric zones have been identified on São Vicente, on morphological grounds. These correspond to geological units that were recently established by Ancochea et al. (2010):
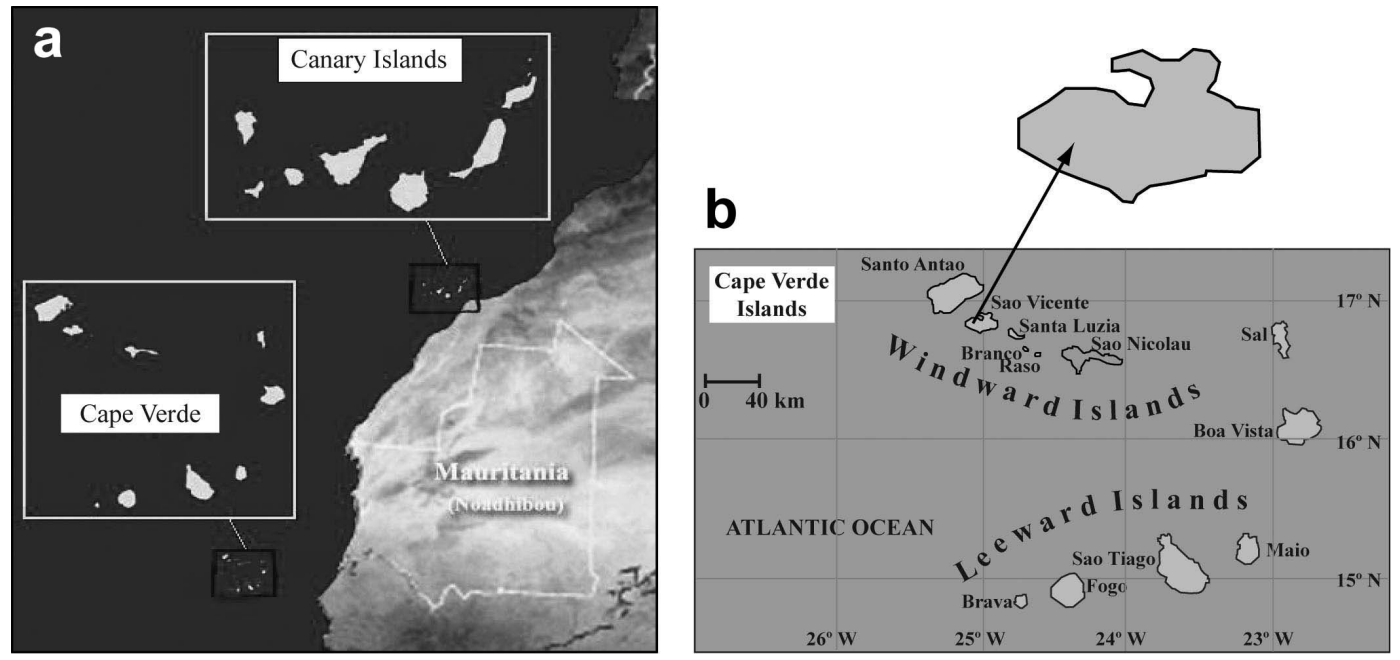

FIG. 1. The Cape Verde Islands. (a) Geographical location of the Cape Verde archipelago. (b) The island of São Vicente which is in the northern part of the archipelago and is located along a NW-SE trend in the Windward Islands. 
(1) The inner zone, occupying the central part of the island, is a flat depression with several rounded hills not exceeding $100-150 \mathrm{~m}$ in elevation, which is typically covered by reddish aeolian sand. This unit was defined as the Complexo Eruptivo Interno Antigo by Serralheiro (1976), who interpreted it as the bottom of a caldera (Cha da Caldeira). Ancochea et al. (2010) define it as the Basal Complex (Submarine Edifice) and interpret it as the submarine stage of the main volcanic edifice on the island. Exposures of intrusive volcanic rock from different stages of formation are present. It is made up of submarine basaltic rocks and intrusive gabbros and syenites, which are cross-cut by a dense dyke swarm that is predominantly basaltic in composition but includes carbonatite dykes. The Basal Complex also contains narrow plugs or remnants of lava flows of phonolitic or pyroxene-nephelinite composition.

(2) A mountain range made up of dipping lava and rare pyroclastic flows, which reaches elevations of 300-700 m, surrounds the inner zone and is dissected by three major valleys that connect the flat central core of the island to the sea (Fig. 2). This mountain range is defined as the Main Volcanic Edifice by Ancochea et al. (2010) who distinguish a lower part, which is $400 \mathrm{~m}$ thick and made up of basaltic lava, commonly ankaramitic pahoehoe flows with some intercalated breccias, and an upper part which is largely nephelinite.

(3) A peripheral zone consists of well preserved pyroclastic cones and some platform-forming lava flows.

\section{Field relations of the carbonatites and nephelinites}

In the central part of the island (Fig. 2), a carbonatite dyke swarm trending roughly northsouth cuts the Basal Complex basaltic dykes and volcanic material of the Submarine Edifice. These dykes are up to $50 \mathrm{~cm}$ wide and a few metres long and crop out along the road connecting Mindelo with Calhau (point A in Fig. 2). The dykes cut the lower part of the Main Volcanic Edifice, indicating that they post-date it; they are related to the upper part of the São Vicente Edifice, which is largely nephelinite.

One of the largest outcrops of carbonatite is located a few kilometres southeast of Mindelo, at Hortas de Camilla, (Fig. 2, point B). It consists of a tabular, subhorizontal body of carbonatite trending northeast-southwest, which is roughly $20 \mathrm{~m}$ long and 4-5 $\mathrm{m}$ wide, and rises about 3-4 $\mathrm{m}$ above the land surface (Fig. $3 a$ ). The carbonatite is very fine grained with centimetrescale banding, alternating from white and brownish yellow to brownish purple (Fig. 3b). Brittle shear structures including small scale faults and pinch-and-swell deformation of the slightly coarser layers are present locally (Fig. 3b, white layer). Ubiquitous vertical jointing in the outcrop is coupled with conjugate subhorizontal joints, defining lobes, which are interpreted as overlapping flow lobes. This structure could be a sill, but on the one hand there are no signs of country rock in the surroundings, and on the other in the central part of the body, the banding is highly convoluted (Fig. 3c), resembling very fluid lava. Taking this into account, the outcrop is interpreted as a carbonatite lava flow.

North of Hortas de Camilla (point C in Fig. 2), there are carbonatite outcrops that are similar to the one described above (Fig. $3 d, e$ ). Some have a more granular, though still fine-grained texture (Fig. 3d), suggesting they represent either a subvolcanic facies, which could correspond to feeder dykes, or a recrystallized extrusive carbonatite.

Distinctive outcrops, similar to those described above, rising 3-4 $\mathrm{m}$ from the ground, and scattered blocks, some resembling volcanic bombs, which are composed of granular, intrusive carbonatite are present over almost all of the area shown in Fig. 2. Small apophyses of intrusive carbonatite are also present, these are interpreted as the eroded remnants of a subvolcanic-extrusive carbonatite system. Many carbonatites worldwide are associated with alkaline silicate rocks, and central São Vicente is no exception. The carbonatite apophyses are spatially related to nephelinite plugs (Fig. 3, point D), which have been dated at $4 \mathrm{Ma}$ (Ancochea et al., 2010). Therefore, this age can also be assigned to the carbonatites. This reinforces the link between the cross-cutting dyke swarm, which includes carbonatite dykes, and the upper part of the São Vicente Edifice.

Nephelinites are abundant at Areia Branca, east of Mindelo (Fig. 3, point E), where carbonatites are present as $\mathrm{cm}$-sized veins and a few dykes. Here nephelinite lavas are interspersed with carbonatite lenses, showing that the same conduits might have been used by both kinds of magma. 

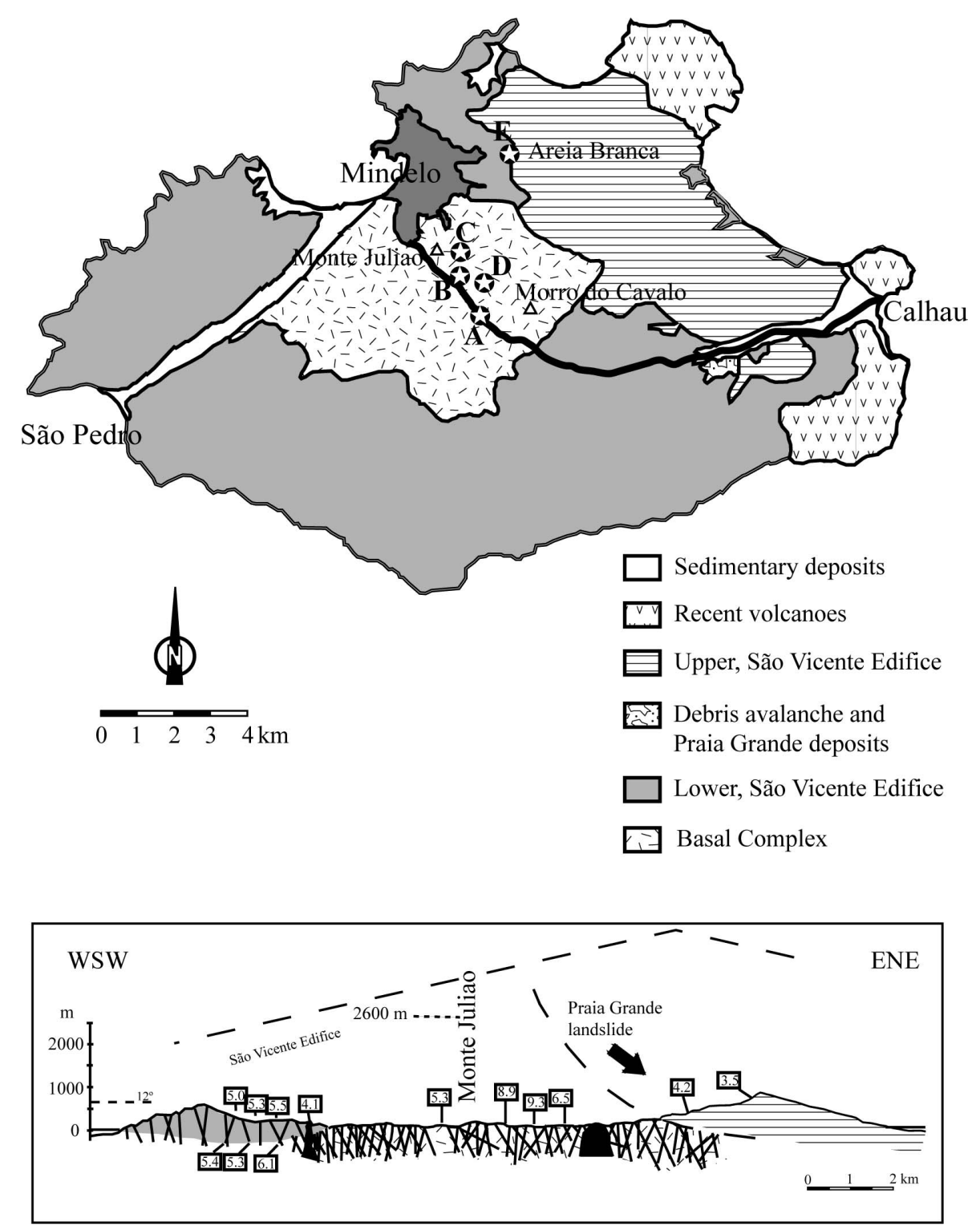

FIG. 2. A simplified geological map and cross-section of São Vicente (redrawn and modified from Ancochea et al., 2010) showing the main units, morphological features, radiometric ages and field relations. Points A to E are selected carbonatite and nephelinite outcrops which are described in the text.

\section{Sampling and analytical techniques}

Both types of carbonatite and nephelinite were sampled. Several carbonatite samples were discarded due to the abundance of secondary 'caliche' (crustose calcium carbonate) alteration. The carbonatite samples are as follows: a very fresh sample from the Hortas de Camilla lava flow (SV-499); a fresh dyke from the dyke swarm cropping out along the Mindelo-Calhau road (SV-538); a carbonatite vein (SV-625), and a sample of nephelinite plus carbonatite lenses (SV-524) from Areia Branca; four samples of 

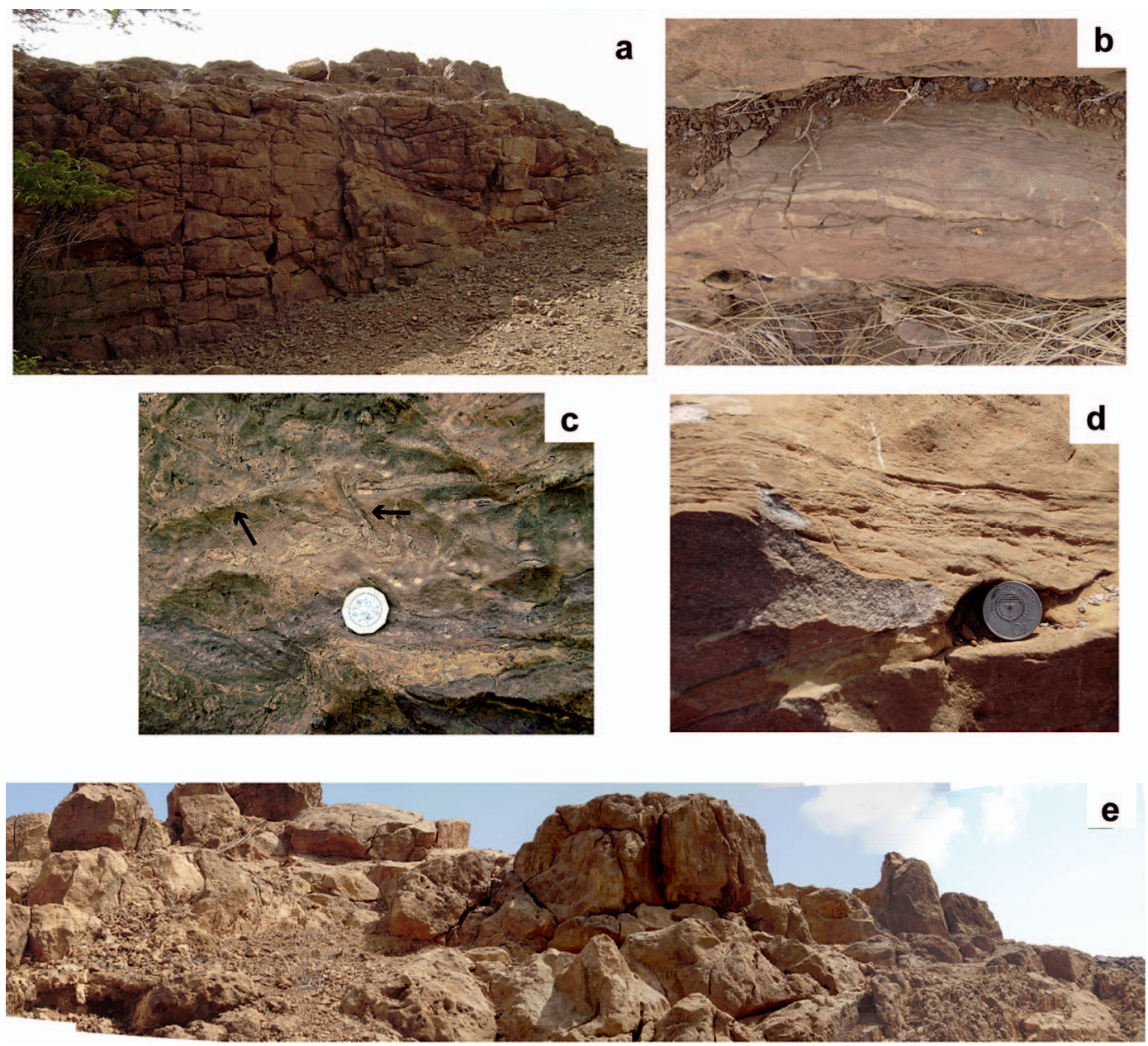

Fig. 3. Field relations of the São Vicente carbonatites. (a) General view of extrusive carbonatite at Hortas de Camilla. The outcrop is $\sim 20 \mathrm{~m}$ long and $5 \mathrm{~m}$ high. (b) Detail of the structure of the extrusive carbonatite at the lateral margins of the lava flow (the field of view is about $20 \mathrm{~cm}$ across); the banding is marked by alternating white and brownish yellow to brownish purple layers. (c) Convoluted banding in the central part of the lava flow (the coin for scale is about the size of a 2 euro coin). (d) and (e) A detailed and field view, respectively, of outcrops to the north of Hortas de Camilla, where carbonatites are either recrystallized extrusive facies or subvolcanic facies.

recrystallized or subvolcanic carbonatite (SV-520; SV-521; SV-523 and SV-525); five samples of intrusive carbonatite (SV-529; SV-530; SV-531; SV-535 and SV-536) and a sample of a nephelinite plug (SV-537).

Chemical analyses were performed by electron probe microanalysis (EPMA), at the Centro de Microscopía Electrónica Luis Bru of the Universidad Complutense de Madrid (Spain). A JEOL Superprobe JXA-8900M, with four spectro- meters and TAP, LD2, PETJ, LIF and LIFH crystal analysers was used. The operating conditions were a $15 \mathrm{kV}$ accelerating potential with a probe current of $20 \mathrm{nA}$. To minimize $\mathrm{Na}_{2} \mathrm{O}$ loss in the feldspathoids and hydrous minerals, sodium was determined first. In the pyrochlore analyses, $\mathrm{HfO}_{2}, \mathrm{Ta}_{2} \mathrm{O}_{5}$, REE, $\mathrm{Y}_{2} \mathrm{O}_{3}, \mathrm{UO}_{2}, \mathrm{ThO}_{2}$ and $\mathrm{PbO}$ were also analysed, and the operating conditions were altered to $20 \mathrm{kV}$ and $50 \mathrm{nA}$. The electron beam diameter was typically $5 \mu \mathrm{m}$, but 
exceptionally it was set to $1 \mu \mathrm{m}$ in the analysis of tiny inclusions or crystals. Counting times were between 10 and $60 \mathrm{~s}$. The ZAF correction procedure was used to reduce the data. Errors are on the order of 2\% (relative) for most elements. Standards are listed in Jarosevitch et al. (1980), Jarosevitch and Boatner (1991) and McGuire et al. (1992), and also include some commercial ones.

Whole-rock chemical analyses were made at Activation Laboratories (Actlabs) in Canada. Major elements were determined by inductively coupled plasma atomic emission spectrometry ICP-AES, and trace elements by inductively coupled plasma mass spectrometry ICP-MS. Sample were prepared by fusion with lithium metaborate/tetraborate, and subsequent digestion for $30 \mathrm{~min}$ in a weak (5\%) nitric acid solution containing an internal standard. The analyses were performed using a standard protocol

Strontium and neodymium isotope compositions were determined at the Centro de Geocronología y Geoquímica Isotópica of the Universidad Complutense de Madrid (Spain). Sample preparation procedures and measurement conditions are described by de Ignacio et al. (2006). Lead isotope compositions were determined at ActLabs. Samples with $\mathrm{Pb}$ concentrations greater than $15 \mathrm{ppm}$ were analysed by ICPMS, those with lesser concentrations were analysed by thermal ionization mass spectrometry (TIMS).

\section{Textures and mineral chemistry \\ Carbonatites}

Petrographic study of the carbonatites allowed us to distinguish three main textural types: (1) banded, extrusive carbonatites and their related dykes, which are rich both in calcite and dolomite; (2) recrystallized, extrusive carbonatites, with only moderate dolomite contents; (3) granular intrusive carbonatites, which are exclusively calcite carbonatites.

\section{Extrusive carbonatites and dykes}

The extrusive carbonatites and dykes have similar textures, but are distinguished at outcrop as the banded structure is subhorizontal in the extrusive carbonatites and subvertical in the dykes. The banding is marked by mm-sized trails made up of microcrystalline aggregates of brownish black dolomite with accessory anhedral mica. Scanning electron microscopy (SEM) reveals the dolomite trails alternate with $0.1-0.3 \mathrm{~mm}$ wide bands composed of microcrystalline apatite and $0.2-0.3 \mathrm{~mm}$ wide calcite bands (Fig. 4). The microcrystalline calcite is tabular and euhedral, and the crystals tend to be orientated with their longest axes parallel to the banding, which we interpret as magmatic flow orientation in the subhorizontal banded structures. The thin dolomite trails resemble structures described by Zaitsev and Polezhaeva (1994), which are thought to be produced by the exsolution of dolomite from early magnesiumrich calcite. In the São Vicente extrusive carbonatites and dykes, dolomite which progressively exsolved and segregated from magnesiumrich calcite merged to form narrow bands with an orientation consistent with that of the calcite. The carbonatites contain accessory pyrochlore, which occasionally cuts the banding (Fig. $5 a$ ), abundant interstitial baryte and rare hollandite (Table 1). The banding has good continuity in sample SV-499 where the dolomite is deep black. The dolomite layers in sample SV-538 are more discontinuous (Fig. $5 b$ ) and two types of dolomite are present: dolomite in the bands, which is dark brown to black, and large dolomite crystals that cut across the banding (Fig. 5c).

The carbonate compositions of the extrusive carbonatites and dykes are listed in Table 2. The

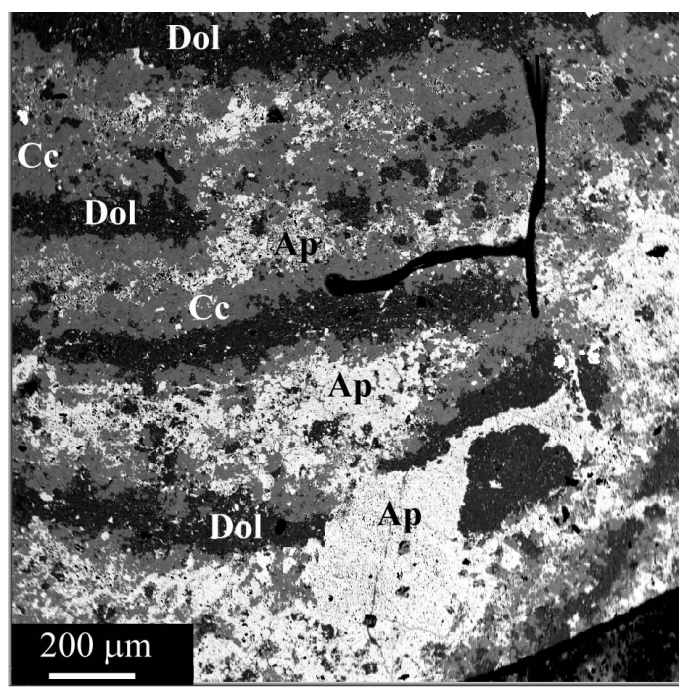

FIG. 4. Back-scattered electron image showing the banded texture in the São Vicente extrusive carbonatites and dykes. Abbreviations are: Ap = apatite (white); Dol $=$ dolomite (dark grey); Cc = calcite (light grey) . 

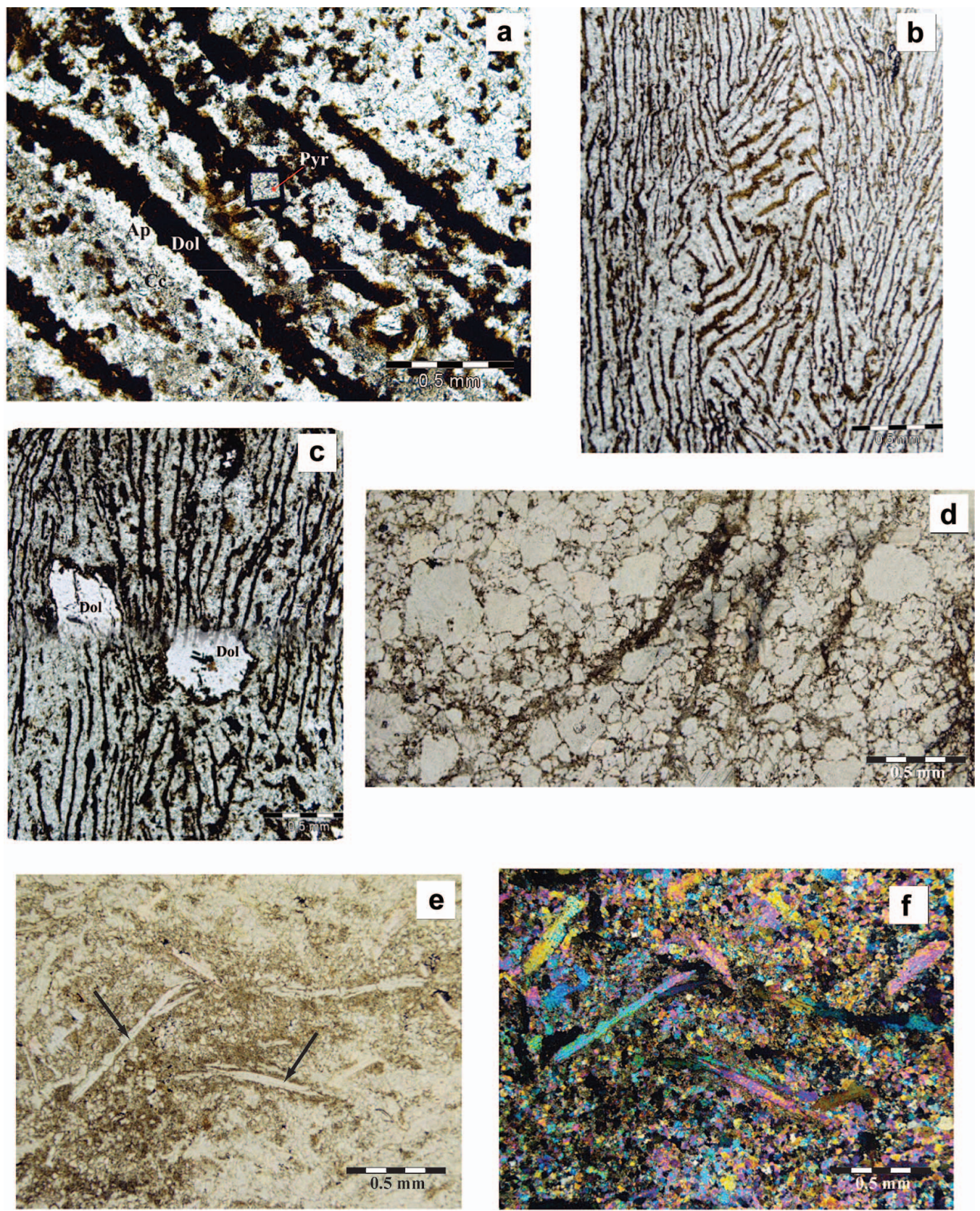

Fig. 5. The main textures of the São Vicente carbonatites (photographs are taken in plane-polarized light unless otherwise stated). (a) Detail of the modal banding consisting of alternate layers of dolomite (Dol), apatite (Ap) and calcite $(\mathrm{Cc})$ with euhedral pyrochlore $(\mathrm{Pyr})$ cutting the bands. In $(b)$ and $(c)$ more discontinuous bands in the carbonatite dykes are shown and two kinds of dolomite are present. (b) Brownish-black dolomite forming part of the bands. (c) Isolated white dolomite crystals cutting the bands. (d) A cataclastic, slightly recrystallized texture in extrusive carbonatite (dolomite is interstitial or makes up the remnants of former bands). (e) and ( $f$ ) Elongated calcite crystals (indicated by black arrows) surrounded by fine-grained calcite, giving the rock an apparently porphyritic texture; (e) is in plane-polarized light and $(f)$ between crossed polarizers. 
TABLE 1. Qualitative modal compositions of samples of carbonatite and nephelinite from São Vicente.

\begin{tabular}{|c|c|c|c|c|c|c|c|c|c|c|}
\hline & $\begin{array}{c}\text { SV-499 } \\
\text { EC }\end{array}$ & $\begin{array}{c}\text { SV-538 } \\
\text { Dyke }\end{array}$ & $\begin{array}{c}\text { SV-521 } \\
\text { REC }\end{array}$ & $\begin{array}{c}\text { SV-625 } \\
\text { REC }\end{array}$ & $\begin{array}{c}\text { SV-523 } \\
\text { REC }\end{array}$ & $\begin{array}{c}\text { SV-529 } \\
\text { IC }\end{array}$ & $\begin{array}{c}\text { SV-531 } \\
\text { IC }\end{array}$ & $\begin{array}{c}\text { SV-536 } \\
\text { IC }\end{array}$ & $\begin{array}{l}\text { SV-537 } \\
\text { NE }\end{array}$ & $\begin{array}{l}\text { SV-524 } \\
\text { NE }\end{array}$ \\
\hline Calcite & $* * * *$ & $* * * *$ & $* * * *$ & $* * * *$ & $* * * *$ & $* * * *$ & $* * * *$ & $* * * *$ & $*$ & - \\
\hline Dolomite & $* * * *$ & $* * * *$ & $* * * *$ & $* * *$ & $* * *$ & - & - & $*$ & $*$ & - \\
\hline Apatite & $* * * *$ & $* * * *$ & $* * * *$ & $* * * *$ & $* * * *$ & $* * * *$ & $* * * *$ & $* * * *$ & ** & - \\
\hline Pyrochlore & $*$ & $*$ & - & - & - & - & - & - & - & - \\
\hline Mica & * & * & - & - & - & $* *$ & $* *$ & $* *$ & $* * *$ & - \\
\hline Clinopyroxene & - & - & - & - & - & $* * *$ & $* * *$ & $* * *$ & $* * * *$ & $* * * *$ \\
\hline Nepheline & - & - & - & - & - & - & - & - & $* *$ & - \\
\hline Amphibole & - & - & - & - & - & - & - & - & ** & - \\
\hline Fe-(Ti) oxides & - & $*$ & $*$ & - & - & * & * & - & $*$ & - \\
\hline Baryte & $* * *$ & ** & ** & ** & $* *$ & - & - & - & - & - \\
\hline Hollandite & - & $*$ & - & $*$ & $*$ & - & - & - & - & - \\
\hline Rhönite & - & - & - & - & - & - & - & - & - & $* *$ \\
\hline
\end{tabular}

Abbreviations are: $\mathrm{EC}=$ extrusive carbonatite; $\mathrm{REC}=$ recrystallized extrusive carbonatite; $\mathrm{IC}=$ intrusive carbonatite; $\mathrm{NE}=$ nephelinite.

The abundance of each mineral phase indicated as follows: $* * * *=$ very abundant; $* * *=$ abundant; $* *=$ present; $*$ accessory; $-=$ absent.

calcite and dolomite bands are made up of very fine grained equidimensional crystals (Fig. 4), the composition of which is similar in both types (extrusive and dykes) of carbonatite. The calcite is pure, with moderate $\mathrm{SrO}(0.2-0.4$ wt. $\%)$ and $\mathrm{FeO}(0.2-0.4$ wt. $\%)$ contents, and $\sim 0.2$ wt. $\%$ $\mathrm{MnO}$. The magnesium content is generally low $(0.2-0.3$ wt. $\% \mathrm{MgO})$ although occasionally it reaches as much as 1 wt.\%. In contrast, the dolomite in the bands is remarkably rich in iron (3-9 wt.\% FeO), and it has a dark brown to black colour under the optical microscope. The dolomite typically contains $\sim 1$ wt. $\% \mathrm{MnO}$ (Table 2); its strontium content is similar to, or lower than, that of the calcite. Zaitsev and Polezhaeva (1994) reported iron-rich, manganese-bearing dolomite from early-stage Kovdor carbonatites, and noted a similar partitioning of elements between the calcite and dolomite (i.e. more $\mathrm{Fe}+\mathrm{Mn}$ and less $\mathrm{Sr}$ in dolomite than in calcite).

Apatite co-crystallized with calcite and dolomite, and forms part of the bands. It has the same habit (equidimensional, with very fine-grained crystals) as the carbonates (Fig. 4). The crystalline shapes and texture could reflect crystallization in a highly supersaturated environment, where many nuclei were present, as Walter et al. (1995) suggest for apatite in the Juquiá Complex, Brazil. Apatite in the São Vicente extrusive carbonatites and dykes is strontium-rich (3 wt.\% $\mathrm{SrO})$ fluorapatite, containing $1.1-2.6$ wt. $\% \mathrm{Na}_{2} \mathrm{O}$ (Table 3). Substitution of $\mathrm{Na}$ into the apatite structure reflects the abundance of the belovite component: $\mathrm{NaREESr}{ }_{3}\left(\mathrm{PO}_{4}\right)_{3} \mathrm{~F}$, which would explain the low analytical totals. This type of apatite is rare in carbonatites. The composition of apatite in the extrusive carbonatites and dykes is very similar to that of early primary apatite from the carbonatites of the Juquiá Complex, Brazil (Walter et al., 1995; Table 3). Co-crystallization of sodium- and strontium-rich apatite with calcite and dolomite in the São Vicente extrusive carbonatites and dykes might provide an explanation of the low $\mathrm{Sr}$ content in the calcite and dolomite in the bands, as $\mathrm{Sr}$ would preferentially partition into apatite. The primary nature suggested by the apatite composition is consistent with the primary nature indicated by the dolomite composition and texture.

In addition to its occurrence as one of the main phases in the bands, apatite is also found as isolated crystals (Table 3), which have lower $\mathrm{Na}_{2} \mathrm{O}(\sim 0.5$ wt.\%), SrO $(0.4$ wt.\%) and $\mathrm{F}$ $(1.7-1.8$ wt.\%) contents, and contain small amounts of $\mathrm{SiO}_{2}(0.5-0.8$ wt.\%). These chemical differences are interpreted as resulting from the later crystallization of this apatite, after the calcite, dolomite and apatite that formed the bands. The presence of $\mathrm{SiO}_{2}$ is consistent with 
TABLE 2. Representative compositions of calcite and dolomite from the extrusive carbonatites and dykes.

\begin{tabular}{|c|c|c|c|c|c|c|c|c|c|c|}
\hline $\begin{array}{l}\text { Oxide } \\
\text { (wt.\%) }\end{array}$ & $\begin{array}{c}\text { SV-499 } \\
\text { Calcite, } \\
\text { bands }\end{array}$ & $\begin{array}{l}\text { SV-499 } \\
\text { Calcite, } \\
\text { bands }\end{array}$ & $\begin{array}{c}\text { SV-499 } \\
\text { Dolo- } \\
\text { mite, } \\
\text { bands }\end{array}$ & $\begin{array}{c}\text { SV-499 } \\
\text { Dolo- } \\
\text { mite, } \\
\text { bands }\end{array}$ & $\begin{array}{l}\text { SV-538 } \\
\text { Calcite, } \\
\text { bands }\end{array}$ & $\begin{array}{c}\text { SV-538 } \\
\text { Calcite, } \\
\text { bands }\end{array}$ & $\begin{array}{c}\text { SV-538 } \\
\text { Dolo- } \\
\text { mite, } \\
\text { disc. } \\
\text { bands }\end{array}$ & $\begin{array}{c}\text { SV-538 } \\
\text { Dolo- } \\
\text { mite, } \\
\text { disc. } \\
\text { bands }\end{array}$ & $\begin{array}{c}\text { SV-538 } \\
\text { Dolo- } \\
\text { mite, } \\
\text { large } \\
\text { crystal }\end{array}$ & $\begin{array}{c}\text { SV-538 } \\
\text { Dolo- } \\
\text { mite, } \\
\text { large } \\
\text { crystal }\end{array}$ \\
\hline $\mathrm{SiO}_{2}$ & 0.00 & 0.00 & 0.00 & 0.00 & 0.00 & 0.00 & 0.00 & 0.00 & 0.00 & 0.00 \\
\hline $\mathrm{TiO}_{2}$ & 0.00 & 0.00 & 0.12 & 0.32 & 0.00 & 0.00 & 0.00 & 0.00 & 0.00 & 0.00 \\
\hline $\mathrm{Al}_{2} \mathrm{O}_{3}$ & 0.00 & 0.02 & 0.00 & 0.20 & 0.00 & 0.00 & 0.00 & 0.00 & 0.00 & 0.00 \\
\hline $\mathrm{FeO}$ & 0.20 & 0.33 & 5.10 & 9.15 & 0.30 & 0.43 & 5.98 & 3.28 & 1.31 & 2.19 \\
\hline $\mathrm{MnO}$ & 0.28 & 0.26 & 1.26 & 1.44 & 0.24 & 0.22 & 0.63 & 1.30 & 0.38 & 0.46 \\
\hline $\mathrm{MgO}$ & 0.30 & 1.12 & 17.48 & 16.12 & 0.13 & 0.32 & 17.31 & 17.77 & 19.86 & 20.37 \\
\hline $\mathrm{CaO}$ & 56.42 & 55.40 & 29.80 & 27.26 & 56.41 & 55.81 & 30.40 & 29.37 & 29.66 & 29.27 \\
\hline $\mathrm{Na}_{2} \mathrm{O}$ & 0.04 & 0.06 & 0.15 & 0.11 & 0.01 & 0.02 & 0.10 & 0.24 & 0.03 & 0.02 \\
\hline $\mathrm{BaO}$ & 0.00 & 0.21 & 0.08 & 0.10 & 0.01 & 0.00 & 0.06 & 0.00 & 0.00 & 0.00 \\
\hline $\mathrm{SrO}$ & 0.29 & 0.38 & 0.26 & 0.32 & 0.21 & 0.42 & 0.19 & 0.33 & 0.87 & 0.81 \\
\hline Total & 57.73 & 57.78 & 54.26 & 55.02 & 57.29 & 57.22 & 54.67 & 52.30 & 52.12 & 53.12 \\
\hline \multicolumn{11}{|l|}{ Element } \\
\hline Ti & 0.000 & 0.000 & 0.004 & 0.010 & 0.000 & 0.000 & 0.000 & 0.000 & 0.000 & 0.000 \\
\hline $\mathrm{Al}$ & 0.000 & 0.001 & 0.000 & 0.008 & 0.000 & 0.000 & 0.000 & 0.000 & 0.000 & 0.000 \\
\hline $\mathrm{Fe}^{2+}$ & 0.006 & 0.009 & 0.134 & 0.241 & 0.008 & 0.012 & 0.155 & 0.088 & 0.035 & 0.057 \\
\hline $\mathrm{Mn}$ & 0.008 & 0.007 & 0.033 & 0.038 & 0.007 & 0.006 & 0.017 & 0.035 & 0.010 & 0.012 \\
\hline $\mathrm{Mg}$ & 0.014 & 0.054 & 0.816 & 0.757 & 0.006 & 0.016 & 0.801 & 0.849 & 0.934 & 0.942 \\
\hline $\mathrm{Ca}$ & 1.966 & 1.917 & 1.000 & 0.921 & 1.975 & 1.958 & 1.012 & 1.009 & 1.003 & 0.973 \\
\hline $\mathrm{Na}$ & 0.002 & 0.004 & 0.009 & 0.007 & 0.001 & 0.002 & 0.006 & 0.015 & 0.002 & 0.001 \\
\hline $\mathrm{Ba}$ & 0.000 & 0.003 & 0.001 & 0.001 & 0.000 & 0.000 & 0.001 & 0.000 & 0.000 & 0.000 \\
\hline $\mathrm{Sr}$ & 0.005 & 0.007 & 0.005 & 0.006 & 0.004 & 0.008 & 0.003 & 0.006 & 0.016 & 0.015 \\
\hline Sum & 2.001 & 2.002 & 2.002 & 1.989 & 2.000 & 2.001 & 1.996 & 2.003 & 2.000 & 2.000 \\
\hline
\end{tabular}

The abbreviation disc. is discontinuous.

this interpretation, as silica is usually concentrated in the last stages of crystallization in carbonatite systems (Le Bas and Handley, 1979).

Pyrochlore is ubiquitous in the São Vicente extrusive carbonatites and dykes. It occurs as colourless, euhedral crystals, which commonly cut across the banding. The crystals are not zoned, and their composition (Table 4) is close to the ideal endmember $(\mathrm{Na}, \mathrm{Ca})_{2} \mathrm{Nb}_{2} \mathrm{O}_{6}(\mathrm{OH}, \mathrm{F})$, which is defined by $\mathrm{Nb}+\mathrm{Ta} \geqslant 2 \mathrm{Ti}$ (Hogarth, 1977; Atencio et al., 2010). Hodgson and Le Bas (1992) described pyrochlore from two carbonatites from São Vicente: a calcite carbonatite from the Camile dyke and a carbonatite from a sövite dyke. Those pyrochlores displayed colour zoning, from red-brown to colourless, which is correlated with composition; the red-brown zones are rich in uranium, but the colourless zones are depleted in this element. The colourless unzoned pyrochlore in our samples contains no uranium, the total $R E E$ content is low at $0.3-0.7$ wt. $\%$, barium is negligible and $\mathrm{SrO}$ amounts to 1 wt.\% (Table 4). These compositions are similar to those of pyrochlore in carbonatites containing dolomite, calcite and phlogopite from Sallanlatvi, Kola Peninsula, Russia (Subbotin and Subbotina, 2000). However, in comparison to the pyrochlore composition in Hodgson and Le Bas (1992), ours contain substantially more $\mathrm{Na}_{2} \mathrm{O} \quad(\sim 8$ wt.\% compared to $\leqslant 6$ wt. $\%$ ) and less $\mathrm{ZrO}_{2}$ (0.5-1 wt.\% compared to $10 \mathrm{wt} . \%)$.

Relating the composition of pyrochlore-group minerals to the degree of fractionation of the magma from which they crystallized is not straightforward due to the variety of cationic substitutions that are possible. Early pyrochloregroup minerals are generally rich in tantalum, uranium and thorium (Subbotin and Subbotina, 


$$
\text { C. DE IGNACIO ET AL. }
$$

TABLE 3. Selected analyses of apatite from the extrusive carbonatites and dykes.

\begin{tabular}{|c|c|c|c|c|c|c|c|c|c|}
\hline $\begin{array}{l}\text { Oxide } \\
\text { wt. } \%\end{array}$ & $\begin{array}{c}\text { SV-499 } \\
\text { Bands }\end{array}$ & $\begin{array}{l}\text { SV-499 } \\
\text { Bands }\end{array}$ & $\begin{array}{c}\text { SV-538 } \\
\text { Bands }\end{array}$ & $\begin{array}{c}\text { SV-538 } \\
\text { Bands }\end{array}$ & $\begin{array}{c}\text { SV-523 } \\
\text { Band } \\
\text { remnant }\end{array}$ & $\begin{array}{c}\text { SV-521 } \\
\text { Band } \\
\text { remnant }\end{array}$ & $\begin{array}{l}\text { SV-521 } \\
\text { Isolated } \\
\text { crystal }\end{array}$ & $\begin{array}{c}\text { SV-521 } \\
\text { Isolated } \\
\text { crystal }\end{array}$ & $\begin{array}{l}\text { Primary } \\
\text { Juquiá }\end{array}$ \\
\hline $\mathrm{SiO}_{2}$ & 0.00 & 0.00 & 0.02 & 0.00 & 0.00 & 0.00 & 0.52 & 0.77 & 0.13 \\
\hline $\mathrm{FeO}$ & 0.29 & 0.78 & 0.58 & 0.68 & 0.19 & 0.17 & 0.05 & 0.07 & 0.31 \\
\hline $\mathrm{MnO}$ & 0.05 & 0.24 & 0.10 & 0.38 & 0.07 & 0.00 & 0.04 & 0.00 & 0.12 \\
\hline $\mathrm{MgO}$ & 0.01 & 0.66 & 0.67 & 0.55 & 0.00 & 0.00 & 0.00 & 0.00 & 0.07 \\
\hline $\mathrm{CaO}$ & 53.05 & 46.48 & 47.34 & 45.06 & 52.58 & 53.90 & 56.02 & 55.94 & 49.69 \\
\hline $\mathrm{Na}_{2} \mathrm{O}$ & 1.16 & 2.58 & 2.87 & 2.24 & 1.69 & 1.47 & 0.44 & 0.55 & 2.36 \\
\hline $\mathrm{K}_{2} \mathrm{O}$ & 0.00 & 0.00 & 0.06 & 0.07 & 0.00 & 0.00 & 0.00 & 0.00 & 0.00 \\
\hline $\mathrm{BaO}$ & 0.08 & 0.43 & 0.34 & 0.33 & 0.06 & 0.00 & 0.13 & 0.00 & 0.20 \\
\hline $\mathrm{SrO}$ & 3.04 & 3.35 & 2.80 & 2.48 & 2.32 & 1.95 & 0.46 & 0.41 & 1.15 \\
\hline $\mathrm{Nb}_{2} \mathrm{O}_{5}$ & 0.00 & 0.00 & 0.00 & 0.14 & 0.00 & 0.00 & 0.00 & 0.00 & n.a. \\
\hline $\mathrm{P}_{2} \mathrm{O}_{5}$ & 37.84 & 34.22 & 32.80 & 35.26 & 37.28 & 38.47 & 38.60 & 37.72 & 37.61 \\
\hline $\mathrm{SO}_{3}$ & 0.28 & 0.00 & 0.00 & 0.22 & 1.10 & 0.97 & 0.10 & 0.11 & 0.02 \\
\hline $\mathrm{F}$ & 3.55 & 2.99 & 2.82 & 2.67 & 2.84 & 3.08 & 1.83 & 1.72 & 2.60 \\
\hline $\mathrm{F}=\mathrm{O}$ & -1.50 & -1.26 & -1.19 & -1.12 & -1.20 & -1.30 & -0.77 & -0.72 & \\
\hline Total & 97.85 & 90.47 & 89.20 & 88.95 & 96.93 & 98.71 & 97.42 & 96.57 & 94.52 \\
\hline \multicolumn{10}{|l|}{ Element } \\
\hline $\mathrm{Fe}$ & 0.040 & 0.111 & 0.081 & 0.101 & 0.023 & 0.026 & 0.007 & 0.010 & - \\
\hline $\mathrm{Mn}$ & 0.007 & 0.035 & 0.013 & 0.058 & 0.000 & 0.010 & 0.006 & 0.000 & - \\
\hline $\mathrm{Mg}$ & 0.002 & 0.167 & 0.168 & 0.146 & 0.000 & 0.000 & 0.000 & 0.000 & - \\
\hline $\mathrm{Ca}$ & 9.290 & 8.476 & 8.499 & 8.613 & 9.334 & 9.205 & 9.797 & 9.778 & - \\
\hline $\mathrm{Na}$ & 0.368 & 0.851 & 0.931 & 0.776 & 0.461 & 0.535 & 0.139 & 0.174 & - \\
\hline K & 0.000 & 0.000 & 0.013 & 0.015 & 0.000 & 0.000 & 0.000 & 0.000 & - \\
\hline $\mathrm{Ba}$ & 0.005 & 0.029 & 0.022 & 0.023 & 0.000 & 0.004 & 0.008 & 0.000 & - \\
\hline $\mathrm{Sr}$ & 0.288 & 0.331 & 0.272 & 0.256 & 0.183 & 0.220 & 0.044 & 0.039 & - \\
\hline $\mathrm{Nb}$ & 0.000 & 0.000 & 0.000 & 0.011 & 0.000 & 0.000 & 0.000 & 0.000 & - \\
\hline Sum & 10.000 & 10.000 & 10.000 & 10.000 & 10.000 & 10.000 & 10.000 & 10.000 & - \\
\hline $\mathrm{P}$ & 5.880 & 5.794 & 5.769 & 5.792 & 5.789 & 5.755 & 5.839 & 5.772 & - \\
\hline $\mathrm{Si}$ & 0.000 & 0.000 & 0.003 & 0.000 & 0.000 & 0.000 & 0.093 & 0.139 & - \\
\hline $\mathrm{S}$ & 0.039 & 0.000 & 0.000 & 0.032 & 0.129 & 0.151 & 0.013 & 0.015 & - \\
\hline $\mathrm{C}^{*}$ & 0.081 & 0.206 & 0.228 & 0.176 & 0.081 & 0.094 & 0.054 & 0.074 & - \\
\hline Sum & 6.000 & 6.000 & 6.000 & 6.000 & 6.000 & 6.000 & 6.000 & 6.000 & - \\
\hline F & 1.105 & 1.029 & 0.991 & 0.919 & 0.945 & 0.892 & 0.057 & 0.544 & - \\
\hline $\mathrm{OH}^{\dagger}$ & 0.895 & 0.971 & 1.009 & 1.080 & 1.055 & 1.108 & 1.430 & 1.456 & - \\
\hline Sum & 2.000 & 2.000 & 2.000 & 2.000 & 2.000 & 2.000 & 2.000 & 2.000 & - \\
\hline
\end{tabular}

Apatite structural formulae were calculated on the basis of $25 \mathrm{O}^{2-}(\mathrm{OH}, \mathrm{F}, \mathrm{Cl})$ following the normalization procedure of Seifert et al. (2000).

$\mathrm{No} \mathrm{Cl}$ or $\mathrm{Zr}$ was detected in any of the apatite analyses.

The SV-523 and SV-521 apatite samples are from recrystallized extrusive carbonatites.

* Primary Juquiá is data for primary apatite from the Juquiá Complex in Brazil from Walter et al. (1995).

* $\mathrm{C}$ the carbon content is calculated on the basis of charge balance, assuming 50 charges (corresponding to 24 oxygen atoms) at the $A+T$ sites.

${ }^{\dagger} \mathrm{OH}$ was calculated by difference, once the other anions were set.

2000; Chakhmouradian and Williams, 2004). These are followed, in a typical crystallization sequence, by pyrochlore sensu stricto and then by low-temperature or hydrothermal pyrochlores which are typically rich either in rare earth elements or in barium and strontium. These 
latter pyrochlores are usually formed by supergene alteration of primary pyrochlore.

In Fig. 6, the fields of primary and progressively altered pyrochlores defined by Nasraoui and Bilal (2000) and Williams et al. (2007) for the alkaline complexes of Lueshe and Bingo (Congo) are shown. The São Vicente pyrochlore compositions plot close to, or inside, the field of magmatic pyrochlore (Fig. 6), and their mineral chemistry is consistent with a primary origin. Only the A2 composition of Hodgson and Le Bas (1992), which is described as an alteration rim, is close to the field of hydrothermal pyrochlores.

In view of the textural relations between pyrochlore and calcite-dolomite-apatite bands in the São Vicente extrusive carbonatites and dykes, the bands can be considered to be composed of early magmatic phases, which suggests they crystallized at high temperatures.

The remaining minerals in the extrusive carbonatites and dykes include mica and interstitial phases. Mica is an accessory mineral in both the Hortas de Camilla extrusive carbonatite (sample SV-499) and in the carbonatite dyke sample (SV-538). However, its composition is different in each of these rocks.
In the extrusive carbonatite sample from Hortas de Camilla (SV-499), mica forms small colourless plates showing no pleochroism. It is always associated with dolomite or apatite in the bands but unlike these minerals, the mica is anhedral and appears resorbed, sometimes being partly replaced by very fine grained dolomite (Fig. 7). This mica has an unusually high silica content (57-58 wt. $\% \mathrm{SiO}_{2}$ ), practically no aluminium, a high magnesium content (19-20 wt.\% MgO) and a high fluorine content (3.6-3.8 wt.\%); its analytical totals are always low (Table 5). These features are similar to those of taeniolite, a lithium-rich mica described in carbonatites from Dicker Willem in South Africa by Cooper and Patterson (1995). Although lithium cannot be detected by electron-microprobe analysis, an estimation of the possible content can be made by analysing the total oxygen content of the mica. This yielded $\sim 3 \mathrm{wt} . \%$, which is in good agreement with the lithium content reported by Cooper and Patterson (1995) in the Dicker Willem carbonatite (Table 5).

The carbonatite dyke sample (SV-538) contains tiny aggregates of a different kind of mica, which has a subhedral, flake-like habit and brown to

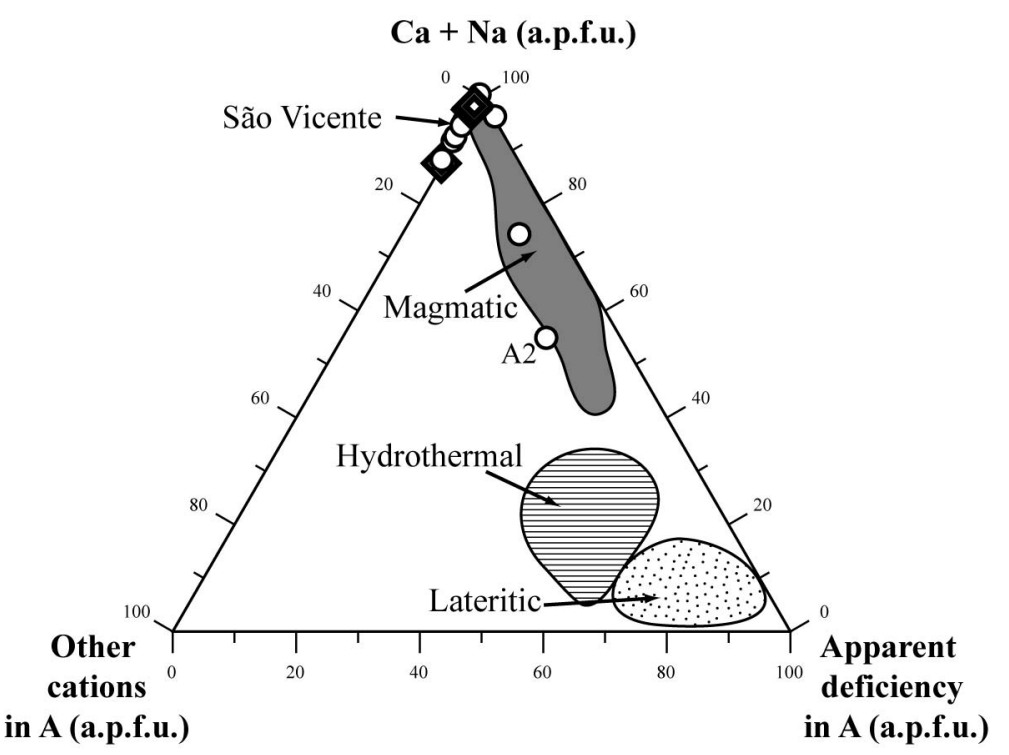

FIG. 6. A ternary diagram showing the variation in composition between early-crystallized (magmatic) pyrochlore (grey-shaded field), late-stage (hydrothermal) pyrochlore (striped field) and supergene pyrochlore (dotted field). Data for fields were taken from Nasraoui and Bilal (2000) and Williams et al. (1997). The symbols are: circles for the pyrochlore analyses from Hodgson and Le Bas (1992), A2 corresponds to an alteration rim also Hodgson and Le Bas (1992); diamonds for pyrochlore in extrusive carbonatites and dykes from this study. 
TABLE 4. Representative pyrochlore compositions in the extrusive carbonatites and dykes.

\begin{tabular}{lrrrrrr}
\hline Oxide (wt.\%) & SV-499 & SV-499 & SV-499 & SV-499 & SV-499 & SV-499 \\
\hline $\mathrm{SiO}_{2}$ & 0.16 & 0.23 & 0.14 & 0.20 & 0.21 & 0.44 \\
$\mathrm{TiO}_{2}$ & 5.34 & 5.15 & 5.12 & 5.18 & 4.68 & 5.57 \\
$\mathrm{Al}_{2} \mathrm{O}_{3}$ & 0.00 & 0.02 & 0.05 & 0.01 & 0.01 & 0.03 \\
$\mathrm{FeO}$ & 0.10 & 0.11 & 0.12 & 0.09 & 0.10 & 0.20 \\
$\mathrm{MnO}$ & 0.01 & 0.02 & 0.02 & 0.00 & 0.01 & 0.03 \\
$\mathrm{MgO}$ & 0.01 & 0.00 & 0.00 & 0.01 & 0.01 & 0.01 \\
$\mathrm{CaO}$ & 19.76 & 19.64 & 19.61 & 19.75 & 18.91 & 19.94 \\
$\mathrm{Na} 2$ & 8.37 & 8.77 & 8.42 & 8.72 & 8.63 & 8.39 \\
$\mathrm{BaO}$ & 0.08 & 0.07 & 0.09 & 0.06 & 0.08 & 0.08 \\
$\mathrm{SrO}$ & 0.98 & 0.98 & 1.01 & 1.00 & 1.19 & 0.90 \\
$\mathrm{ZrO}_{2}$ & 0.80 & 0.92 & 0.91 & 0.76 & 0.56 & 1.30 \\
$\mathrm{HfO}_{2}$ & 0.00 & 0.04 & 0.02 & 0.03 & 0.00 & 0.03 \\
$\mathrm{Ta}_{2} \mathrm{O}_{5}$ & 0.13 & 0.13 & 0.11 & 0.10 & 0.09 & 0.15 \\
$\mathrm{Nb}_{2} \mathrm{O}_{5}$ & 62.21 & 62.77 & 62.61 & 62.55 & 63.07 & 61.36 \\
$\mathrm{Y}_{2} \mathrm{O}_{3}$ & 0.07 & 0.06 & 0.08 & 0.05 & 0.07 & 0.07 \\
$\mathrm{La}_{2} \mathrm{O}_{3}$ & 0.17 & 0.15 & 0.16 & 0.15 & 0.19 & 0.17 \\
$\mathrm{Ce}_{2} \mathrm{O}_{3}$ & 0.34 & 0.33 & 0.35 & 0.10 & 0.32 & 0.40 \\
$\mathrm{Pr}_{2} \mathrm{O}_{3}$ & 0.03 & 0.01 & 0.02 & 0.00 & 0.01 & 0.02 \\
$\mathrm{Nd}_{2} \mathrm{O}_{3}$ & 0.08 & 0.06 & 0.06 & 0.06 & 0.08 & 0.07 \\
$\mathrm{UO}_{2}$ & 0.00 & 0.00 & 0.00 & 0.00 & 0.00 & 0.00 \\
$\mathrm{ThO}_{2}$ & 0.17 & 0.20 & 0.19 & 0.19 & 0.10 & 0.24 \\
$\mathrm{PbO}_{\mathrm{F}}$ & 0.04 & 0.03 & 0.01 & 0.08 & 0.05 & 0.03 \\
$\mathrm{~F} \mathrm{O}$ & 2.00 & 1.97 & 1.92 & 2.20 & 1.96 & 1.96 \\
$\mathrm{Total}$ & -0.84 & -0.83 & -0.81 & -0.93 & -0.83 & -0.82 \\
$\mathrm{Fe}_{2} \mathrm{O}_{3}$ & 99.97 & 100.82 & 100.20 & 100.35 & 99.49 & 100.53 \\
$\mathrm{Total}^{*}$ & 0.11 & 0.12 & 0.14 & 0.10 & 0.11 & 0.22 \\
$\Sigma R E E^{\dagger}$ & 100.08 & 100.94 & 100.33 & 100.45 & 99.60 & 100.75 \\
& 0.61 & 0.56 & 0.59 & 0.31 & 0.60 & 0.65
\end{tabular}

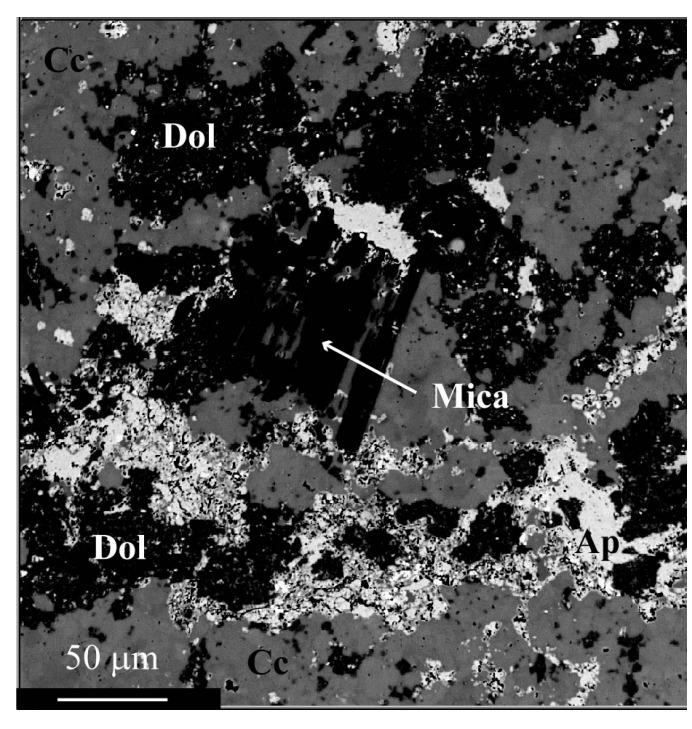

reddish pleochroism. This mica (Table 5) is phlogopite, with $\sim 1$ wt.\% fluorine and a very low titanium content $\left(0.3-0.4\right.$ wt. $\% \quad \mathrm{TiO}_{2}$, Table 5). The very low analytical totals may be due to excess water.

The $\mathrm{Fe}-\mathrm{Ti}$ oxides in the carbonatite dyke include Ti-bearing to Ti-free magnetites, which have relatively high manganese contents (up to 5 wt. $\% \mathrm{MnO}$, Table 6). Magnesium is present at much lower concentrations $(0.2-0.9$ wt. $\% \mathrm{MgO})$. Ilmenite has a relatively low iron content (23-28 wt.\% FeO) and low analytical totals, which suggest alteration. It has $0.4-0.6$ wt. $\%$ $\mathrm{MnO}$ and around 1 wt.\% $\quad \mathrm{Nb}_{2} \mathrm{O}_{5}$ (Table 6).

Fig. 7. Back-scattered electron image showing the textural relations of mica in extrusive carbonatites (sample SV-499). Abbreviations are: $\mathrm{Cc}=$ calcite (light grey); $\mathrm{Ap}=$ apatite (white); Dol = dolomite (dark grey). 
TABLE 4 (contd.).

\begin{tabular}{|c|c|c|c|c|c|c|}
\hline Oxide (wt.\%) & SV-499 & SV-499 & SV-499 & SV-499 & SV-499 & SV-499 \\
\hline \multicolumn{7}{|l|}{ Element (ppm) } \\
\hline $\mathrm{Mn}$ & 0.000 & 0.001 & 0.001 & 0.000 & 0.001 & 0.001 \\
\hline Mg & 0.001 & 0.000 & 0.000 & 0.001 & 0.001 & 0.001 \\
\hline $\mathrm{Ca}$ & 1.122 & 1.107 & 1.113 & 1.115 & 1.081 & 1.124 \\
\hline $\mathrm{Na}$ & 0.860 & 0.895 & 0.865 & 0.891 & 0.893 & 0.856 \\
\hline $\mathrm{Ba}$ & 0.002 & 0.001 & 0.002 & 0.001 & 0.002 & 0.002 \\
\hline $\mathrm{Sr}$ & 0.030 & 0.030 & 0.031 & 0.030 & 0.037 & 0.027 \\
\hline $\mathrm{U}$ & 0.000 & 0.000 & 0.000 & 0.000 & 0.000 & 0.000 \\
\hline Th & 0.002 & 0.002 & 0.002 & 0.002 & 0.001 & 0.003 \\
\hline $\mathrm{Pb}$ & 0.001 & 0.000 & 0.000 & 0.001 & 0.001 & 0.000 \\
\hline Y & 0.002 & 0.002 & 0.002 & 0.001 & 0.002 & 0.002 \\
\hline $\mathrm{La}$ & 0.003 & 0.003 & 0.003 & 0.003 & 0.004 & 0.003 \\
\hline $\mathrm{Ce}$ & 0.007 & 0.006 & 0.007 & 0.001 & 0.006 & 0.008 \\
\hline $\operatorname{Pr}$ & 0.001 & 0.000 & 0.000 & 0.000 & 0.000 & 0.000 \\
\hline $\mathrm{Nd}$ & 0.001 & 0.001 & 0.001 & 0.001 & 0.001 & 0.001 \\
\hline Total & 2.031 & 2.049 & 2.026 & 2.050 & 2.030 & 2.028 \\
\hline $\mathrm{Si}$ & 0.008 & 0.012 & 0.008 & 0.010 & 0.011 & 0.023 \\
\hline $\mathrm{Ti}$ & 0.213 & 0.204 & 0.204 & 0.205 & 0.188 & 0.221 \\
\hline Al & 0.000 & 0.001 & 0.003 & 0.000 & 0.000 & 0.002 \\
\hline $\mathrm{Fe}^{3+}$ & 0.004 & 0.005 & 0.005 & 0.004 & 0.004 & 0.009 \\
\hline $\mathrm{Zr}$ & 0.021 & 0.024 & 0.023 & 0.020 & 0.015 & 0.033 \\
\hline $\mathrm{Hf}$ & 0.000 & 0.001 & 0.000 & 0.000 & 0.000 & 0.001 \\
\hline Та & 0.002 & 0.002 & 0.002 & 0.001 & 0.001 & 0.002 \\
\hline $\mathrm{Nb}$ & 1.491 & 1.493 & 1.499 & 1.490 & 1.522 & 1.460 \\
\hline Sum & 1.739 & 1.740 & 1.744 & 1.732 & 1.741 & 1.750 \\
\hline
\end{tabular}

The pyrochlore structural formulae are calculated on the basis of $6 \mathrm{O}^{2-}$.

* The $\mathrm{Fe}_{2} \mathrm{O}_{3}$ value gives total iron as $\mathrm{Fe}(\mathrm{III})$.

* the second total following conversion of total iron to $\mathrm{Fe}(\mathrm{III})$.

$+\Sigma R E E$ is the total rare earth content from $\mathrm{La}_{2} \mathrm{O}_{3}$ to $\mathrm{Nd}_{2} \mathrm{O}_{3}$.

Attempts to determine the temperature and oxygen fugacity using magnetite-ilmenite pairs did not yield consistent results, which corroborates the ilmenite alteration hypothesis.

The interstitial minerals in the São Vicente extrusive carbonatites and dykes include baryte and hollandite (Table 7). The baryte is relatively pure and contains very little strontium (Table 7). The hollandite contains 55-57 wt.\% $\mathrm{MnO}$ and $11-12$ wt. $\% \mathrm{BaO}$, a composition that is typical of cryptomelane-group manganese oxides, which are commonly formed by weathering. The hollandite is probably secondary, and may have formed by weathering and alteration of the Mn-bearing, band-forming dolomite.

\section{Recrystallized extrusive carbonatites}

Carbonatites described in the field as possible subvolcanic or recrystallized extrusive facies are mostly dykes and only occasionally occur as deposits with subhorizontal stacking structures that are interpreted as volcanic in origin. However, in this case, secondary textural alteration partially masks the original extrusive texture. These carbonatites are heterogranular, medium- to finegrained rocks. Thin elongated secondary calcite crystals are surrounded by fine-grained calcite, producing a porphyritic texture (Fig. $5 e, f$ ). Dolomite is typically late-stage and is concentrated in small fissures and cracks. The rest of the mineralogy is very similar to that of the extrusive carbonatites and dykes (Table 1), and it includes apatite, magnetite and interstitial baryte and hollandite. Considering their field relations, texture and modal mineralogy, we interpret the carbonatites as recrystallized extrusive carbonatites.

The calcite has a low strontium $(0.2-0.5$ wt. $\%$ $\mathrm{SrO}$, Table 8$)$, iron (0.2 wt. $\% \mathrm{FeO})$ and 
C. DE IGNACIO ET AL.

TABLE 5. Compositional variations in the accessory mica-group minerals from the extrusive carbonatites and dykes.

\begin{tabular}{|c|c|c|c|c|c|c|}
\hline Oxide (wt.\%) & SV-499 & SV-499 & SV-499 & SV-538 & SV-538 & SV-538 \\
\hline $\mathrm{SiO}_{2}$ & 58.66 & 57.40 & 58.04 & 39.10 & 39.78 & 40.34 \\
\hline $\mathrm{TiO}_{2}$ & 0.17 & 0.01 & 0.12 & 0.37 & 0.35 & 0.39 \\
\hline $\mathrm{Al}_{2} \mathrm{O}_{3}$ & 0.51 & 0.01 & 0.38 & 7.99 & 8.10 & 9.21 \\
\hline $\mathrm{FeO}$ & 0.40 & 0.72 & 0.49 & 0.00 & 0.00 & 7.05 \\
\hline $\mathrm{MnO}$ & 0.00 & 0.03 & 0.02 & 0.00 & 0.01 & 0.13 \\
\hline $\mathrm{MgO}$ & 19.83 & 19.48 & 20.21 & 19.23 & 19.72 & 21.89 \\
\hline $\mathrm{CaO}$ & 0.12 & 0.97 & 0.02 & 0.05 & 0.16 & 0.15 \\
\hline $\mathrm{Na}_{2} \mathrm{O}$ & 0.09 & 0.18 & 0.13 & 0.19 & 0.18 & 0.23 \\
\hline $\mathrm{K}_{2} \mathrm{O}$ & 11.29 & 10.89 & 11.38 & 9.63 & 9.77 & 9.87 \\
\hline $\mathrm{NiO}$ & 0.05 & 0.08 & 0.05 & 0.00 & 0.00 & 0.04 \\
\hline $\mathrm{Cr}_{2} \mathrm{O}_{3}$ & 0.01 & 0.00 & 0.01 & 0.17 & 0.35 & 0.13 \\
\hline $\mathrm{F}$ & 6.61 & 6.17 & 6.26 & 1.70 & 2.01 & 2.08 \\
\hline $\mathrm{F}=\mathrm{O}$ & -2.79 & -2.60 & -2.64 & -0.72 & -0.85 & -0.88 \\
\hline $\mathrm{Cl}$ & 0.02 & 0.01 & 0.03 & 0.04 & 0.02 & 0.01 \\
\hline $\mathrm{Cl}=\mathrm{O}$ & -0.00 & -0.00 & -0.01 & -0.01 & -0.00 & -0.00 \\
\hline $\mathrm{SO}_{3}$ & 0.00 & 0.00 & 0.00 & 0.01 & 0.01 & 0.06 \\
\hline Total & $94.98^{*}$ & 93.34 & 94.52 & 77.77 & 79.62 & 90.70 \\
\hline \multicolumn{7}{|l|}{ Element (ppm) } \\
\hline $\mathrm{Si}$ & 8.281 & 8.269 & 8.241 & 6.712 & 6.690 & 6.204 \\
\hline $\mathrm{Al}^{\mathrm{IV}}$ & 0.000 & 0.000 & 0.000 & 1.288 & 1.310 & 1.670 \\
\hline Sum & 8.281 & 8.269 & 8.241 & 8.000 & 8.000 & 7.874 \\
\hline $\mathrm{Al}^{\mathrm{VI}}$ & 0.085 & 0.001 & 0.063 & 0.330 & 0.296 & 0.000 \\
\hline $\mathrm{Ti}$ & 0.018 & 0.001 & 0.013 & 0.048 & 0.044 & 0.045 \\
\hline $\mathrm{Cr}$ & 0.001 & 0.000 & 0.001 & 0.024 & 0.047 & 0.016 \\
\hline $\mathrm{Mg}$ & 4.172 & 4.183 & 4.276 & 4.921 & 4.942 & 5.016 \\
\hline $\mathrm{Fe}^{2+}$ & 0.047 & 0.087 & 0.059 & 0.000 & 0.000 & 0.907 \\
\hline $\mathrm{Mn}$ & 0.000 & 0.003 & 0.002 & 0.000 & 0.001 & 0.017 \\
\hline $\mathrm{Ni}$ & 0.006 & 0.009 & 0.006 & 0.000 & 0.000 & 0.005 \\
\hline Sum & 4.329 & 4.283 & 4.419 & 5.323 & 5.330 & 6.005 \\
\hline $\mathrm{Ca}$ & 0.018 & 0.150 & 0.003 & 0.010 & 0.029 & 0.025 \\
\hline $\mathrm{Na}$ & 0.025 & 0.050 & 0.035 & 0.063 & 0.060 & 0.067 \\
\hline K & 2.034 & 2.002 & 2.062 & 2.109 & 2.095 & 1.927 \\
\hline Sum & 2.077 & 2.203 & 2.102 & 2.182 & 2.184 & 2.029 \\
\hline Total cations & 14.687 & 14.755 & 14.760 & 15.404 & 15.514 & 15.908 \\
\hline $\mathrm{Mg} /\left(\mathrm{Mg}+\mathrm{Fe}^{2+}\right)$ & 0.989 & 0.980 & 0.986 & 1.000 & 1.000 & 0.847 \\
\hline
\end{tabular}

Structural formulae were calculated based on $22 \mathrm{O}^{2-}(\mathrm{OH}, \mathrm{F}, \mathrm{Cl})$ with all iron as $\mathrm{Fe}$ (II).

* This analysis includes $3 \mathrm{wt} . \% \mathrm{Li}_{2} \mathrm{O}$, calculated from the total oxygen content in mica determined by EPMA, and 1.5 wt. $\% \mathrm{H}_{2} \mathrm{O}$ to charge balance the total oxygen.

magnesium (0.1 wt. \% $\mathrm{MgO})$ content. Large calcite crystals sometimes show patchy zonation when examined using back-scattered electron imaging, with light patches enriched in strontium (1 wt.\% SrO, Table 8). The elongated calcite crystals typically contain 0.5 wt. $\%$ SrO, $0.3-0.5$ wt. $\% \mathrm{MnO}$ and $<0.5 \mathrm{MgO}$ wt. $\%$, with occasional patches of Sr-rich calcite $(1.4 \mathrm{wt} . \%$ $\mathrm{SrO}$, Table 8). If the patches are excluded, the compositions of the elongated calcite crystals 
TABLE 6. Fe-Ti oxide mineral compositions in the extrusive carbonatites and dykes.

\begin{tabular}{|c|c|c|c|c|c|c|}
\hline Oxide (wt.\%) & $\begin{array}{c}\text { SV-538 } \\
\text { Magnetite }\end{array}$ & $\begin{array}{c}\text { SV-538 } \\
\text { Magnetite }\end{array}$ & $\begin{array}{c}\text { SV-538 } \\
\text { Magnetite }\end{array}$ & $\begin{array}{c}\text { SV-538 } \\
\text { Magnetite }\end{array}$ & $\begin{array}{l}\text { SV-538 } \\
\text { Ilmenite }\end{array}$ & $\begin{array}{l}\text { SV-538 } \\
\text { Ilmenite }\end{array}$ \\
\hline $\mathrm{SiO}_{2}$ & 0.33 & 0.16 & 0.19 & 0.46 & 0.21 & 0.90 \\
\hline $\mathrm{TiO}_{2}$ & 2.71 & 0.03 & 0.51 & 1.46 & 59.53 & 60.63 \\
\hline $\mathrm{Al}_{2} \mathrm{O}_{3}$ & 0.89 & 0.25 & 0.14 & 0.46 & 0.02 & 0.11 \\
\hline $\mathrm{FeO}$ & 74.30 & 76.64 & 71.30 & 77.61 & 27.97 & 23.47 \\
\hline $\mathrm{MnO}$ & 0.71 & 1.02 & 5.33 & 0.65 & 0.41 & 0.61 \\
\hline $\mathrm{MgO}$ & 0.22 & 0.92 & 0.35 & 0.21 & 0.10 & 0.09 \\
\hline $\mathrm{CaO}$ & 0.66 & 0.28 & 0.36 & 0.92 & 0.32 & 0.82 \\
\hline $\mathrm{NiO}$ & 0.02 & 0.00 & 0.00 & 0.01 & 0.03 & 0.01 \\
\hline $\mathrm{Cr}_{2} \mathrm{O}_{3}$ & 0.08 & 0.06 & 0.11 & 0.08 & 0.10 & 0.14 \\
\hline $\mathrm{Nb}_{2} \mathrm{O}_{5}$ & 0.00 & 0.00 & 0.00 & 0.00 & 1.78 & 1.41 \\
\hline Total & 79.91 & 79.36 & 78.28 & 81.86 & 90.46 & 88.18 \\
\hline $\mathrm{Fe}_{2} \mathrm{O}_{3}$ & 51.82 & 58.57 & 56.50 & 56.11 & 0.00 & 0.00 \\
\hline $\mathrm{FeO}$ & 27.67 & 23.94 & 20.46 & 27.12 & 27.97 & 23.47 \\
\hline Total* & 85.10 & 85.23 & 83.95 & 87.48 & 90.46 & 86.78 \\
\hline \multicolumn{7}{|l|}{ Element (ppm) } \\
\hline $\mathrm{Si}$ & 0.115 & 0.057 & 0.069 & 0.160 & 0.072 & 0.318 \\
\hline $\mathrm{Ti}$ & 0.725 & 0.008 & 0.138 & 0.381 & 15.423 & 16.040 \\
\hline $\mathrm{Al}$ & 0.373 & 0.103 & 0.060 & 0.189 & 0.009 & 0.047 \\
\hline $\mathrm{Fe}^{3+}$ & 13.843 & 15.693 & 15.378 & 14.664 & 0.000 & 0.000 \\
\hline $\mathrm{Fe}^{2+}$ & 8.215 & 7.129 & 6.190 & 7.876 & 8.058 & 6.904 \\
\hline $\mathrm{Mn}$ & 0.213 & 0.308 & 1.632 & 0.192 & 0.121 & 0.183 \\
\hline $\mathrm{Mg}$ & 0.115 & 0.490 & 0.190 & 0.109 & 0.051 & 0.048 \\
\hline $\mathrm{Ca}$ & 0.250 & 0.108 & 0.138 & 0.341 & 0.118 & 0.308 \\
\hline $\mathrm{Ni}$ & 0.006 & 0.000 & 0.000 & 0.004 & 0.009 & 0.003 \\
\hline $\mathrm{Cr}$ & 0.022 & 0.016 & 0.033 & 0.023 & 0.027 & 0.038 \\
\hline $\mathrm{Nb}$ & 0.000 & 0.000 & 0.017 & 0.000 & 0.023 & 0.019 \\
\hline Sum & 23.877 & 23.912 & 23.828 & 23.939 & 23.911 & 23.908 \\
\hline
\end{tabular}

Structural formulae were calculated on the basis of $32 \mathrm{O}^{2-}$ for magnetite; $36 \mathrm{O}^{2-}$ for ilmenite. *The total following appropriate recalculation of $\mathrm{Fe}$ (II) and $\mathrm{Fe}(\mathrm{III})$.

resemble those of the band-forming calcite in the Hortas de Camilla extrusive carbonatite and dyke samples. The fine-grained, microgranular calcite forming the matrix in these rocks is also rich in strontium (0.6 wt.\% SrO).

Dolomite occurs as inclusions in the large calcite crystals and also as an interstitial phase. In the first case, the dolomite is iron-rich, with a composition similar to that of the band-forming dolomite from the extrusive Hortas de Camilla carbonatite (sample SV-499). We interpret this dolomite as a remnant of former bands. The interstitial dolomite has a moderate iron content, which is similar to the isolated, late-stage dolomite from the dykes (sample SV-538). The rims of the large calcite crystals and the intergranular spaces are commonly occupied by low-Mg calcite (around 1.7 wt.\% MgO, Table 8), which is cemented by baryte and hollandite. Low$\mathrm{Mg}$ calcite is also found between Na-rich apatite crystals.

Our interpretation of these textural features is that: (1) recrystallization has increased the strontium content in the calcite, probably remobilizing this element from early apatite; and (2) primary dolomite was partially removed, and is only preserved as relics inside the large calcite crystals, this released the magnesium that is present in the secondary low-Mg interstitial calcite and the manganese to form the hollandite.

Recrystallization of the extrusive carbonatites also affected the apatite compositions. In apatite 


$$
\text { C. DE IGNACIO ET AL. }
$$

TABLE 7. Compositions of the interstitial, late-stage and secondary minerals from the extrusive carbonatites and dykes.

\begin{tabular}{lrrrrr}
\hline $\begin{array}{l}\text { Oxide } \\
\text { wt.\% }\end{array}$ & $\begin{array}{c}\text { SV-499 } \\
\text { Baryte }\end{array}$ & $\begin{array}{c}\text { SV-499 } \\
\text { Baryte }\end{array}$ & $\begin{array}{c}\text { SV-538 } \\
\text { Baryte }\end{array}$ & $\begin{array}{c}\text { SV-538 } \\
\text { Hollandite }\end{array}$ & $\begin{array}{c}\text { SV-538 } \\
\text { Hollandite }\end{array}$ \\
\hline $\mathrm{SiO}_{2}$ & 0.05 & 0.00 & 0.07 & 0.40 & 0.22 \\
$\mathrm{TiO}_{2}$ & 0.00 & 0.00 & 0.00 & 0.09 & 0.29 \\
$\mathrm{Al}_{2} \mathrm{O}_{3}$ & 0.18 & 0.14 & 0.13 & 0.17 & 0.13 \\
$\mathrm{FOO}$ & 0.08 & 0.03 & 0.87 & 1.76 & 5.30 \\
$\mathrm{MnO}$ & 0.08 & 0.09 & 0.04 & 56.84 & 55.80 \\
$\mathrm{MgO}$ & 0.71 & 0.00 & 0.07 & 1.62 & 1.57 \\
$\mathrm{CaO}$ & 0.96 & 0.50 & 0.41 & 0.78 & 0.94 \\
$\mathrm{Na} 2$ & 0.19 & 0.14 & 0.18 & 0.35 & 0.62 \\
$\mathrm{~K}_{2} \mathrm{O}$ & 0.03 & 0.00 & 0.01 & 0.26 & 0.45 \\
$\mathrm{BaO}$ & 62.30 & 66.54 & 64.82 & 11.19 & 0.11 \\
$\mathrm{SrO}$ & 0.29 & 0.29 & 0.63 & 0.46 & 0.03 \\
$\mathrm{SO} \mathrm{S}_{3}$ & 35.48 & 32.24 & 32.84 & 0.03 & 78.03 \\
$\mathrm{Total}$ & 100.16 & 99.96 & 100.08 & 73.95 & \\
\hline
\end{tabular}

from the recrystallized carbonatites, sodium is relatively abundant $\left(1.5-1.7\right.$ wt.\% $\quad \mathrm{Na}_{2} \mathrm{O}$, Table 3), whereas strontium is somewhat depleted
( 2 wt.\% SrO), which is consistent with our hypothesis that strontium was incorporated into the recrystallized calcite grains. Some isolated

TABLE 8. Representative compositions of calcite and dolomite from the recrystallized extrusive carbonatites.

\begin{tabular}{|c|c|c|c|c|c|c|c|c|c|}
\hline $\begin{array}{l}\text { Oxide } \\
\text { wt. } \%\end{array}$ & $\begin{array}{c}\text { SV-524 } \\
\text { Calcite, } \\
\text { large } \\
\text { crystal } \\
\text { Core }\end{array}$ & $\begin{array}{c}\text { SV-524 } \\
\text { Calcite, } \\
\text { large } \\
\text { crystal } \\
\text { Light } \\
\text { patch }\end{array}$ & $\begin{array}{l}\text { SV-524 } \\
\text { Micro- } \\
\text { granular } \\
\text { calcite }\end{array}$ & $\begin{array}{c}\text { SV-524 } \\
\text { Dolomite, } \\
\text { in large } \\
\text { calcite }\end{array}$ & $\begin{array}{c}\text { SV-524 } \\
\text { Low-Mg } \\
\text { calcite } \\
\text { Inter- } \\
\text { granular }\end{array}$ & $\begin{array}{l}\text { SV-625 } \\
\text { Calcite, } \\
\text { elongate } \\
\text { crystal }\end{array}$ & $\begin{array}{c}\text { SV-625 } \\
\text { Calcite, } \\
\text { elongate } \\
\text { crystal } \\
\text { Light } \\
\text { patch }\end{array}$ & 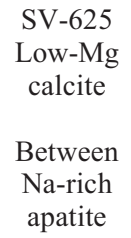 & $\begin{array}{c}\text { SV-625 } \\
\text { Dolomite, } \\
\text { intersti- } \\
\text { tial }\end{array}$ \\
\hline $\mathrm{FeO}$ & 0.16 & 0.08 & 0.06 & 5.96 & 1.74 & 0.09 & 0.08 & 0.05 & 1.50 \\
\hline $\mathrm{MnO}$ & 0.03 & 0.00 & 0.00 & 0.10 & 0.05 & 0.28 & 0.27 & 0.04 & 0.66 \\
\hline $\mathrm{MgO}$ & 0.13 & 0.08 & 0.03 & 14.23 & 1.72 & 0.46 & 0.20 & 1.74 & 17.59 \\
\hline $\mathrm{CaO}$ & 54.34 & 54.11 & 53.88 & 30.75 & 51.63 & 53.05 & 53.12 & 53.58 & 30.84 \\
\hline $\mathrm{Na}_{2} \mathrm{O}$ & 0.03 & 0.03 & 0.00 & 0.22 & 0.04 & 0.01 & 0.01 & 0.06 & 0.19 \\
\hline $\mathrm{BaO}$ & 0.05 & 0.00 & 0.00 & 0.01 & 0.01 & 0.00 & 0.12 & 0.00 & 0.00 \\
\hline $\mathrm{SrO}$ & 0.16 & 0.96 & 0.64 & 0.44 & 0.09 & 0.55 & 1.38 & 0.24 & 0.23 \\
\hline Total & 54.91 & 55.25 & 54.61 & 51.72 & 55.30 & 54.45 & 55.20 & 55.71 & 55.30 \\
\hline \multicolumn{10}{|c|}{ Element (ppm) } \\
\hline $\mathrm{Fe}^{2+}$ & 0.003 & 0.006 & 0.002 & 0.214 & 0.063 & 0.003 & 0.003 & 0.002 & 0.052 \\
\hline $\mathrm{Mn}$ & 0.000 & 0.001 & 0.000 & 0.004 & 0.002 & 0.010 & 0.010 & 0.002 & 0.023 \\
\hline $\mathrm{Mg}$ & 0.003 & 0.005 & 0.001 & 0.509 & 0.062 & 0.017 & 0.017 & 0.062 & 0.612 \\
\hline $\mathrm{Ca}$ & 1.973 & 1.982 & 1.984 & 1.100 & 1.858 & 1.954 & 1.949 & 1.903 & 1.073 \\
\hline $\mathrm{Na}$ & 0.002 & 0.002 & 0.000 & 0.015 & 0.003 & 0.001 & 0.001 & 0.004 & 0.013 \\
\hline $\mathrm{Ba}$ & 0.000 & 0.002 & 0.000 & 0.000 & 0.000 & 0.000 & 0.004 & 0.000 & 0.000 \\
\hline $\mathrm{Sr}$ & 0.035 & 0.006 & 0.024 & 0.016 & 0.003 & 0.020 & 0.051 & 0.008 & 0.008 \\
\hline Sum & 2.016 & 2.004 & 2.011 & 1.857 & 1.993 & 2.006 & 2.027 & 1.981 & 1.782 \\
\hline
\end{tabular}




\section{CARBONATITES AND NEPHELINITES, SÃO VICENTE, CAPE VERDE}

apatite crystals are very depleted in both elements (0.4-0.6 wt. \% $\mathrm{Na}_{2} \mathrm{O} ; 0.4-0.5$ wt. \% $\mathrm{SrO}$, Table 3).

The other minerals have similar compositions to those described for the extrusive carbonatites and dykes.

\section{Intrusive carbonatites}

The intrusive carbonatites are typical sövites, with a medium- to coarse-grained granular or slightly heterogranular texture (Fig. 8a). The carbonate in them is almost entirely calcite, only one sample (SV-529) contained interstitial accessory dolomite. Grain boundaries between the large calcite crystals are very sharp, with no cements, and therefore we consider these rocks to be calcite adcumulates.

Apatite did not co-crystallize with the carbonates; it seems to have formed after them, as shown by its textural position at calcite grain boundaries. The intrusive carbonatites contain clinopyroxene, which is absent in the extrusive types and dykes. The clinopyroxene is green and pleochroic; it is anhedral with embayments and alteration along fractures. Mica is relatively abundant in the intrusive carbonaties; it has dark rims at its contact with calcite and undulating extinction. Other differences in modal mineralogy between the extrusive carbonatites and dykes and intrusive carbonatites are the absence of pyrochlore in the intrusive carbonatites, and the presence of reaction textures involving clinopyroxene in some samples.

The mineral chemistry of the carbonates in the São Vicente intrusive carbonatites is more straightforward than in the extrusive types and dykes. The large calcite grains have a monotonous composition, they are strontium-rich (0.6-0.8 wt.\% SrO, Table 9), with relatively small variations in their iron, magnesium and manganese content (Table 9). The calcite in sample SV-536 (where dolomite is also present) contains less strontium (around 0.3 wt.\% $\mathrm{SrO}$ ) than the other intrusive samples; the dolomite contains $0.4-0.5 \mathrm{wt} . \% \mathrm{FeO}$ and is interstitial to the calcite.

The apatite in the intrusive carbonatites is different to the apatite in the extrusive carbonatites and dykes. It contains far less sodium $\left(0.1-0.2\right.$ wt.\% $\left.\mathrm{Na}_{2} \mathrm{O}\right)$ and less strontium and fluorine (Table 10). Although the apatite in each sample is not zoned, there are slight variations in composition between the samples, which contain $0.2-0.6$ wt. $\%$ SrO.
The clinopyroxene generally has an aegirineaugite composition (Fig. $9 a$ ), with 5 wt. $\% \mathrm{Na}_{2} \mathrm{O}$ in the cores to $6-7$ wt. $\% \mathrm{Na}_{2} \mathrm{O}$ in the rims (Table 11). It is slightly zoned, with magnesium and calcium decreasing from core to rim, and iron, titanium and aluminium increasing in the same direction (Table 11, Fig. 9a). The clinopyroxene contains $0.1-0.5$ wt. $\% \mathrm{ZrO}_{2}$.

Aegirine-augite in some of the intrusive carbonatites (sample SV-529) has a reaction rim at its contact with the carbonate (Fig. 9b). The rims are made up of tiny needles of almost pure aegirine and almost pure diopside (black stars in Fig. $9 a$ ). The reaction rims might indicate early crystallization of a clinopyroxene with a composition that became unstable as carbonatite crystallization proceeded.

One sample (SV-536) contains clinopyroxene with a sub-silicic Ti-rich diopside-like composition (Table 11; Fig. 9a). It has patchy zoning, with dark patches of diopside and light patches of sodiumand $\mathrm{Fe}$ (III)-rich diopside, which plots near to the aegirine-augite composition field (Fig. 9a).

An examination of their textural relationships suggests that the mica-group minerals are not in chemical equilibrium in all of the samples. The mica in sample SV-529 (Table 12) seems to be closest to equilibrium. It is relatively rich in titanium (3.0-3.5 wt. $\left.\% \mathrm{TiO}_{2}\right)$, with a $\mathrm{Mg} /(\mathrm{Mg}+$ $\left.\mathrm{Fe}^{2+}\right)$ ratio of $0.6-0.7$, and is therefore biotite. It contains a small amount of fluorine $(0.3-0.4$ wt. $\%$ F) and sodium $(0.3-0.4$ wt. $\%$ $\mathrm{Na}_{2} \mathrm{O}$ ). The mica in sample SV-531, and especially in SV-536, contains a considerable amount of magnesium (up to $22 \mathrm{wt} . \% \mathrm{MgO}$ ) but less titanium $\left(\sim 2 \mathrm{wt} . \% \mathrm{TiO}_{2}\right)$, and is therefore phlogopite. The increase in the magnesium content is accompanied by a decrease in iron content; this is especially noticeable in sample SV-536 where the micas have the greatest magnesium contents. In this sample, the micas also have relatively low fluorine contents and low analytical totals, which might indicate slight chloritization.

Accessory iron oxides occur in the intrusive carbonatites as scattered, anhedral crystals. Their compositions are dominated by iron, with no titanium and practically no aluminium (Table 13). The iron oxides appear to contain some silica; this might be due to a matrix effect during analysis (due to their small size), but probably represents a real compositional feature indicating that they formed as late-stage or secondary phases, as a result of the alteration of earlier magnetite. 
C. DE IGNACIO ET AL.
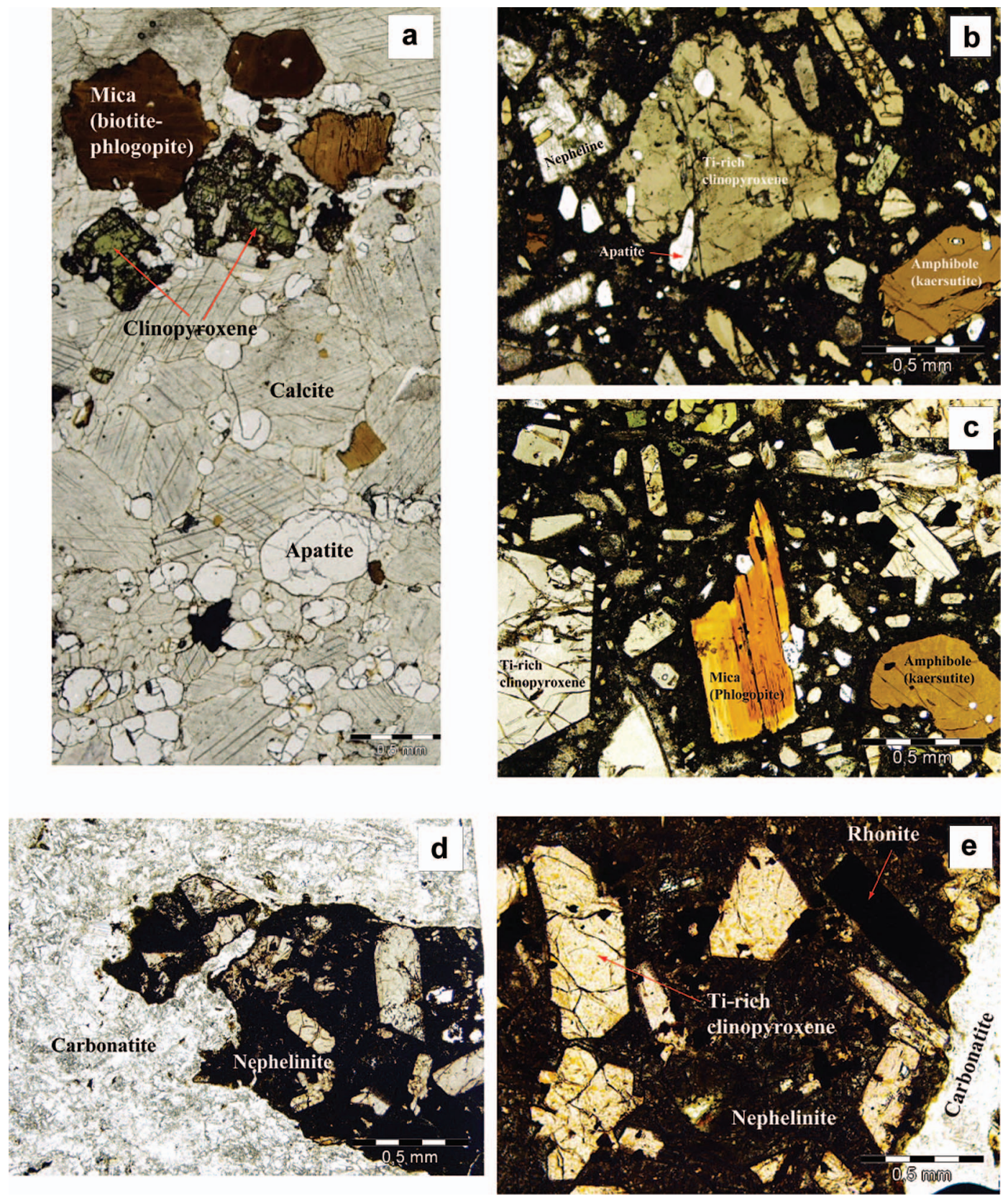

FIG. 8. The main textures of the São Vicente intrusive carbonatites and nephelinites (the photographs are taken in plane-polarized light). (a) Coarse-grained sövite showing large calcite crystals, together with apatite, clinopyroxene, mica and oxide minerals. $(b)$ and $(c)$ Nephelinites forming plugs in the area to the east of Hortas de Camilla. The phenocryst-rich, slightly seriate texture and dominance of clinopyroxene can be seen, together with the occurrence of amphibole and mica. (d) Nephelinite interspersed with carbonatite from Areia Branca. (e) Details of the nephelinite texture and mineralogy: dominant clinopyroxene and rhönite are the only phenocrysts present. 
TABLE 9. Representative compositions of carbonates from the intrusive carbonatites and nephelinites.

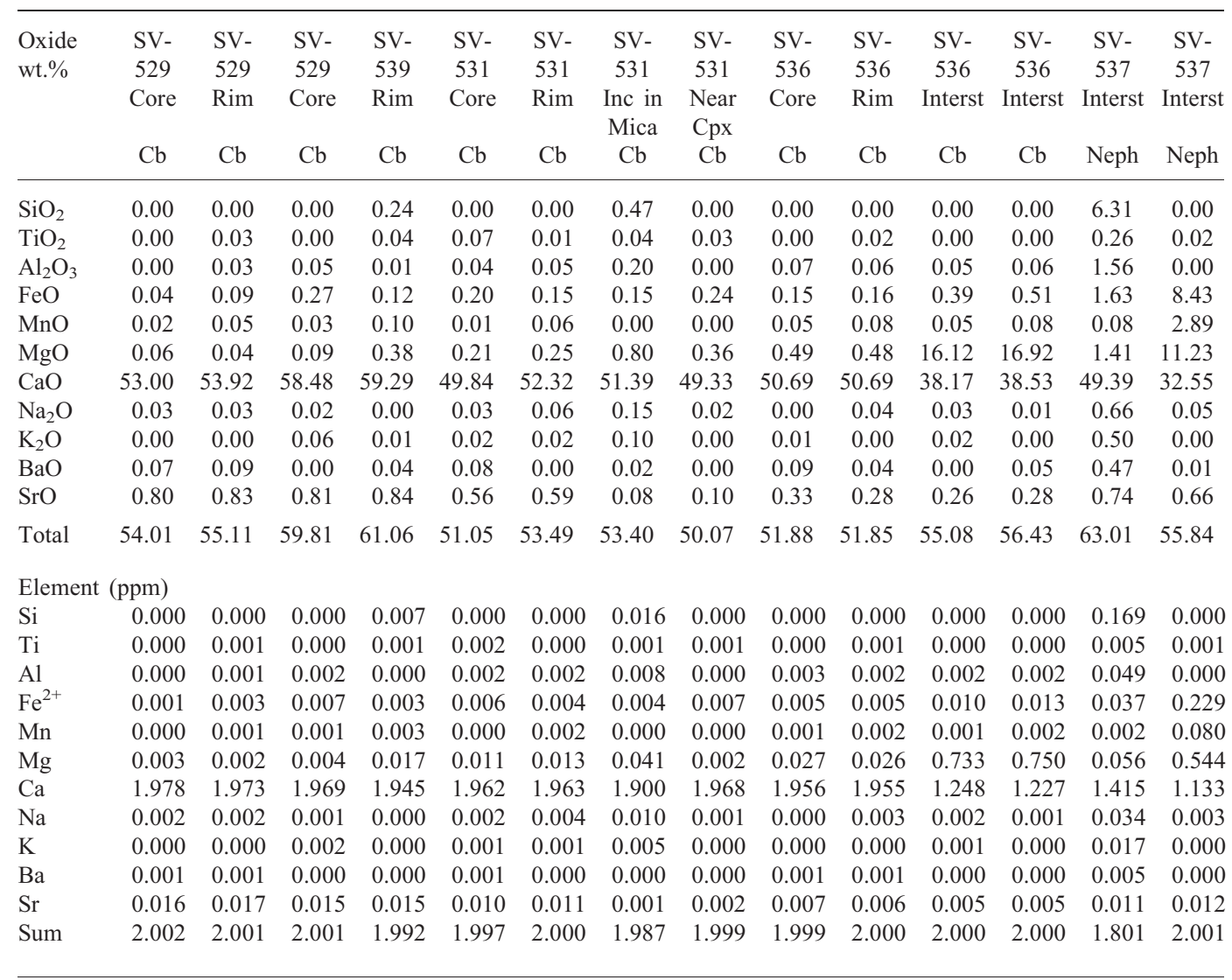

Abbreviations are: $\mathrm{Cb}=$ carbonatite; $\mathrm{Neph}=$ Nephelinite; Inc in mica $=$ inclusion in mica; Interst $=$ Interstitial.

\section{Nephelinites}

Two slightly different types of nephelinite are associated with the carbonatites. Those forming plugs in the area to the east of Hortas de Camilla, where apophyses of intrusive carbonatite crop out, have a strongly porphyritic texture; they are made up of pinkish Ti-rich clinopyroxene, nepheline, apatite, amphibole plus mica and some carbonate, in a microcrystalline to vitreous matrix (Fig. 8b,c). The nephelinites from Areia Branca are also porphyritic, but amphibole- and micagroup minerals are absent; they are made up of pinkish clinopyroxene and rhönite in a vitreous matrix (Fig. 8d,e). Olivine is absent from both types. Clinopyroxene is always the dominant phenocryst; the crystals are euhedral with a variable size $(0.5-2.0 \mathrm{~mm})$, producing porphyritic seriate textures.
The sparse carbonates in the Hortas de Camilla area nephelinite are small interstitial crystals of low-Mg calcite and $\mathrm{Fe}$ - and Mn-rich dolomite (Table 9). These compositions resemble those of carbonates in the extrusive carbonatites and dykes, although the strontium contents in the nephelinite carbonates are high (0.6-0.7 wt.\% SrO).

Apatite in the nephelinites contains small amounts of silica and sulfur, as is typical in alkaline silicate rocks associated with carbonatites (Stoppa and Liu, 1995). The apatite in the nephelinites is otherwise similar in composition to the apatite in the intrusive carbonatites. The difference in the silica content is particularly interesting. The primary apatite in both extrusive and intrusive carbonatites from São Vicente contains no silica, which implies a relatively early origin (crystallization in a silica-free 


$$
\text { C. DE IGNACIO ET AL. }
$$

TABLE 10. Representative compositions of apatite from the intrusive carbonatites and nephelinites.

\begin{tabular}{|c|c|c|c|c|c|c|c|}
\hline $\begin{array}{l}\text { Oxide } \\
\text { wt. } \%\end{array}$ & $\begin{array}{c}\text { SV-529 } \\
\text { Core } \\
\text { Cb }\end{array}$ & $\begin{array}{c}\text { SV-529 } \\
\text { Rim } \\
\text { Cb }\end{array}$ & $\begin{array}{c}\text { SV-531 } \\
\text { Core } \\
\text { Cb }\end{array}$ & $\begin{array}{l}\text { SV-531 } \\
\text { Rim } \\
\text { Cb }\end{array}$ & $\begin{array}{c}\text { SV-536 } \\
\text { Core } \\
\text { Cb }\end{array}$ & $\begin{array}{l}\text { SV-536 } \\
\text { Rim } \\
\text { Cb }\end{array}$ & $\begin{array}{c}\text { SV-537 } \\
\text { Core } \\
\text { Neph }\end{array}$ \\
\hline $\mathrm{SiO}_{2}$ & 0.02 & 0.01 & 0.00 & 0.02 & 0.00 & 0.15 & 0.30 \\
\hline $\mathrm{FeO}$ & 0.12 & 0.06 & 0.01 & 0.00 & 0.04 & 0.02 & 0.20 \\
\hline $\mathrm{MnO}$ & 0.03 & 0.04 & 0.04 & 0.04 & 0.03 & 0.04 & 0.00 \\
\hline $\mathrm{MgO}$ & 0.01 & 0.00 & 0.00 & 0.01 & 0.00 & 0.01 & 0.01 \\
\hline $\mathrm{CaO}$ & 55.83 & 57.01 & 56.27 & 55.68 & 56.08 & 56.40 & 56.36 \\
\hline $\mathrm{Na}_{2} \mathrm{O}$ & 0.17 & 0.28 & 0.18 & 0.21 & 0.22 & 0.19 & 0.15 \\
\hline $\mathrm{K}_{2} \mathrm{O}$ & 0.01 & 0.00 & 0.01 & 0.02 & 0.02 & 0.00 & 0.01 \\
\hline $\mathrm{BaO}$ & 0.00 & 0.00 & 0.12 & 0.06 & 0.02 & 0.00 & 0.12 \\
\hline $\mathrm{SrO}$ & 0.56 & 0.49 & 0.38 & 0.33 & 0.16 & 0.18 & 0.37 \\
\hline $\mathrm{ZrO}_{2}$ & 0.00 & 0.00 & 0.05 & 0.06 & 0.00 & 0.11 & 0.00 \\
\hline $\mathrm{Nb}_{2} \mathrm{O}_{5}$ & 0.08 & 0.08 & 0.07 & 0.06 & 0.05 & 0.11 & 0.02 \\
\hline $\mathrm{P}_{2} \mathrm{O}_{5}$ & 41.97 & 41.62 & 41.98 & 40.84 & 41.67 & 41.40 & 39.69 \\
\hline $\mathrm{SO}_{3}$ & 0.03 & 0.04 & 0.04 & 0.04 & 0.00 & 0.08 & 0.28 \\
\hline $\mathrm{F}$ & 2.30 & 1.89 & 2.23 & 2.08 & 1.43 & 1.31 & 1.59 \\
\hline $\mathrm{F}=\mathrm{O}$ & -0.97 & -0.30 & -0.94 & -0.88 & -0.60 & -0.55 & -0.67 \\
\hline $\mathrm{Cl}$ & 0.02 & 0.01 & 0.00 & 0.04 & 0.02 & 0.01 & 0.12 \\
\hline $\mathrm{Cl}=\mathrm{O}$ & -0.00 & -0.00 & -0.00 & -0.01 & -0.00 & -0.00 & -0.03 \\
\hline Total & 100.17 & 100.71 & 100.43 & 98.60 & 99.11 & 99.44 & 98.51 \\
\hline \multicolumn{8}{|c|}{ Element (ppm) } \\
\hline $\mathrm{Fe}$ & 0.016 & 0.008 & 0.001 & 0.000 & 0.005 & 0.003 & 0.027 \\
\hline $\mathrm{Mn}$ & 0.004 & 0.005 & 0.005 & 0.006 & 0.004 & 0.005 & 0.000 \\
\hline $\mathrm{Mg}$ & 0.003 & 0.000 & 0.000 & 0.002 & 0.000 & 0.002 & 0.002 \\
\hline $\mathrm{Ca}$ & 9.860 & 9.850 & 9.883 & 9.876 & 9.896 & 9.896 & 9.878 \\
\hline $\mathrm{Na}$ & 0.055 & 0.087 & 0.056 & 0.067 & 0.071 & 0.060 & 0.046 \\
\hline K & 0.003 & 0.000 & 0.002 & 0.004 & 0.003 & 0.000 & 0.003 \\
\hline $\mathrm{Ba}$ & 0.000 & 0.000 & 0.008 & 0.004 & 0.001 & 0.000 & 0.008 \\
\hline $\mathrm{Sr}$ & 0.054 & 0.046 & 0.036 & 0.031 & 0.015 & 0.017 & 0.035 \\
\hline $\mathrm{Zr}$ & 0.000 & 0.000 & 0.004 & 0.005 & 0.000 & 0.009 & 0.000 \\
\hline $\mathrm{Nb}$ & 0.006 & 0.005 & 0.005 & 0.005 & 0.003 & 0.008 & 0.001 \\
\hline Sum & 10.000 & 10.000 & 10.000 & 10.000 & 10.000 & 10.000 & 10.000 \\
\hline $\mathrm{P}$ & 5.983 & 5.976 & 5.986 & 5.979 & 5.984 & 5.957 & 5.895 \\
\hline $\mathrm{Si}$ & 0.004 & 0.002 & 0.001 & 0.004 & 0.000 & 0.025 & 0.053 \\
\hline $\mathrm{S}$ & 0.004 & 0.005 & 0.005 & 0.005 & 0.000 & 0.010 & 0.037 \\
\hline $\mathrm{C}^{*}$ & 0.010 & 0.017 & 0.008 & 0.012 & 0.016 & 0.009 & 0.015 \\
\hline Sum & 6.000 & 6.000 & 6.000 & 6.000 & 6.000 & 6.000 & 6.000 \\
\hline F & 0.686 & 0.563 & 0.662 & 0.632 & 0.432 & 0.397 & 0.488 \\
\hline $\mathrm{Cl}$ & 0.003 & 0.001 & 0.000 & 0.008 & 0.004 & 0.002 & 0.026 \\
\hline $\mathrm{OH}^{\dagger}$ & 1.311 & 1.436 & 1.338 & 1.360 & 1.564 & 1.601 & 1.486 \\
\hline Sum & 2.000 & 2.000 & 2.000 & 2.000 & 2.000 & 2.000 & 2.000 \\
\hline
\end{tabular}

Abbreviations are $\mathrm{Cb}=$ Carbonatite; Neph $=$ Nephelinite.

${ }^{*} \mathrm{C}$ the carbon content is calculated on the basis of charge balance, assuming 50 charges (corresponding to 24 oxygen atoms) at the $A+T$ sites.

${ }^{\dagger} \mathrm{OH}$ was calculated by difference, once the other anions were set.

environment occurs at an early stage in carbonatite magmas according to Le Bas and Handley,
1979). The strontium and fluorine contents, although similar in apatite from nephelinites and 


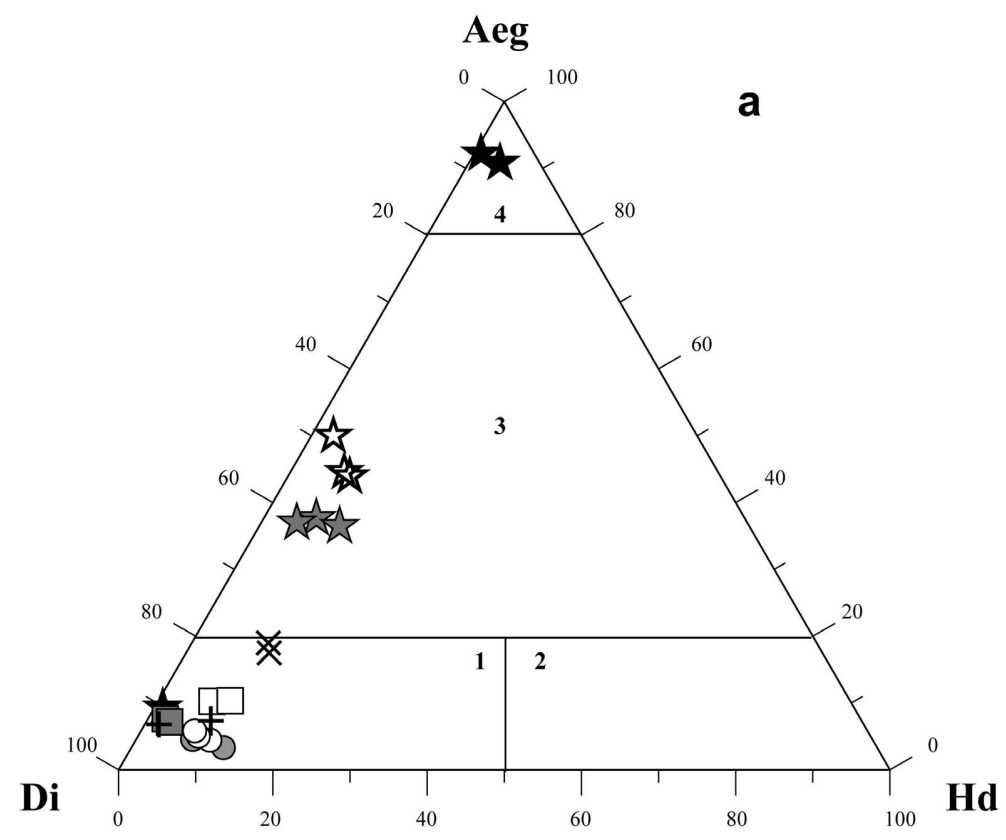

Fig. 9. Textural features and composition of clinopyroxene from the São Vicente intrusive carbonatites and nephelinites. (a) Diagram showing clinopyroxene compositions: field 1 is diopside; field 2 is hedenbergite; field 3 is aegirine-augite; field 4 is aegirine. The symbols for the intrusive carbonatite clinopyroxenes are: grey stars $=$ cores; white stars = rims; black stars $=$ reaction rims with calcite; crosses $=$ dark patches in zoned crystals; $\times$-shapes $=$ light patches in zoned crystals. The symbols for hydrous nephelinites are: grey squares = cores; white squares = rims. The symbols for anhydrous nephelinites are: grey circles = cores; white circles = rims. (b) Back-scattered electron image showing a thin reaction rim between carbonate (calcite, $\mathrm{Cc}$ ) and clinopyroxene (aegirine-augite, Aeg-Aug) in sample

SV-529 (intrusive carbonatite). (c) Concentric zoning in clinopyroxene from the anhydrous nephelinites.

intrusive carbonatites, are very different in the extrusive carbonatites and dykes. In a study of the compositional variation of apatite in the alkalinecarbonatite Vuoriyarvi complex, Brassinnes et al. (2005) observed an increase of strontium, fluorine and light $R E E$ in apatite from progressively more differentiated rocks. If the strontium $v s$. fluorine contents for apatites from the São Vicente carbonatites and associated nephelinites are plotted (Fig. 10), there are two distinct groups: one consisting of the intrusive carbonatites, nephelinites and isolated apatite crystals that occur in some of the recrystallized extrusive carbonatites; and the other consisting of apatites from the bands in extrusive carbonatites and dykes or from band remnants in recrystallized samples. There is a good linear fit to the data in both groups $\left(R^{2}=0.89\right.$, Fig. 10$)$, which is consistent with magmatic differentiation and with the compositional trends described by Brassinnes et al. (2005) for Vuoriyarvi.
Nepheline occurs as small euhedral phenocrysts, which are frequently altered to sodium-rich zeolites. Its composition is homogeneous: $\mathrm{Ne}_{79-80} \mathrm{Ks}_{19-20} \mathrm{Qtz}_{0-1}$. The crystals are not zoned and their calcium content is consistently low (1 wt.\% $\mathrm{CaO}$, Table 14). In the nephelinekalsilite-quartz diagram (Fig. 11), the compositions plot near the field of primary crystallization defined by Henderson and Gibb (1983) and displaced from the line of nepheline co-crystallization with alkali feldspar (the dashed line in Fig. 11). This latter observation is consistent with the sodic character of the magmas from which they crystallized.

The clinopyroxene present in both types of nephelinite is diopside, but there are slight differences in its composition. In the amphibolemica-bearing nephelinite, clinopyroxene zoning from core to rim involves an increase in titanium, iron and sodium, and a decrease in magnesium content. Aluminium either decreases or remains 


\section{DE IGNACIO ET AL.}

TABLE 11. Representative compositions of clinopyroxene from the intrusive carbonatites.

\begin{tabular}{|c|c|c|c|c|c|c|c|c|c|c|c|c|c|}
\hline $\begin{array}{l}\text { Oxide } \\
\text { wt. } \%\end{array}$ & $\begin{array}{c}\text { SV- } \\
529 \\
\text { Core }\end{array}$ & $\begin{array}{l}\text { SV- } \\
529 \\
\text { Rim }\end{array}$ & $\begin{array}{l}\text { SV- } \\
529 \\
\text { Core }\end{array}$ & $\begin{array}{l}\text { SV- } \\
529 \\
\text { Rim }\end{array}$ & $\begin{array}{c}\text { SV- } \\
529 \\
\text { Reaction } \\
\text { rim }\end{array}$ & $\begin{array}{c}\text { SV- } \\
529 \\
\text { Reaction } \\
\text { rim }\end{array}$ & $\begin{array}{c}\text { SV- } \\
529 \\
\text { Reaction } \\
\text { rim }\end{array}$ & $\begin{array}{l}\text { SV- } \\
531 \\
\text { Core }\end{array}$ & $\begin{array}{l}\text { SV- } \\
531 \\
\text { Rim }\end{array}$ & $\begin{array}{c}\text { SV- } \\
536 \\
\text { Dark } \\
\text { patch }\end{array}$ & $\begin{array}{c}\text { SV- } \\
536 \\
\text { Dark } \\
\text { patch }\end{array}$ & $\begin{array}{c}\text { SV- } \\
536 \\
\text { Light } \\
\text { patch }\end{array}$ & $\begin{array}{l}\text { SV- } \\
536 \\
\text { Light } \\
\text { patch }\end{array}$ \\
\hline $\mathrm{SiO}_{2}$ & 52.65 & 52.23 & 53.08 & 52.11 & 52.24 & 51.99 & 52.69 & 53.44 & 53.56 & 52.87 & 53.32 & 52.04 & 52.26 \\
\hline $\mathrm{TiO}_{2}$ & 0.33 & 0.77 & 0.35 & 1.07 & 0.63 & 0.88 & 0.27 & 0.47 & 0.78 & 0.34 & 0.27 & 0.35 & 0.35 \\
\hline $\mathrm{Al}_{2} \mathrm{O}_{3}$ & 0.61 & 1.19 & 0.85 & 1.06 & 0.89 & 0.50 & 0.62 & 0.31 & 0.96 & 0.52 & 0.43 & 1.23 & 1.21 \\
\hline $\mathrm{FeO}$ & 12.90 & 14.42 & 13.40 & 14.32 & 26.79 & 25.98 & 5.38 & 12.81 & 14.57 & 4.59 & 5.01 & 8.74 & 9.12 \\
\hline $\mathrm{MnO}$ & 0.21 & 0.26 & 0.32 & 0.20 & 0.38 & 0.29 & 0.32 & 0.17 & 0.18 & 0.25 & 0.19 & 0.35 & 0.32 \\
\hline $\mathrm{MgO}$ & 9.46 & 8.27 & 9.37 & 8.27 & 0.97 & 1.30 & 14.97 & 10.45 & 8.34 & 15.29 & 14.29 & 11.85 & 11.80 \\
\hline $\mathrm{CaO}$ & 17.06 & 15.19 & 16.58 & 15.40 & 1.77 & 2.07 & 24.37 & 16.39 & 14.08 & 24.79 & 24.73 & 21.84 & 21.64 \\
\hline $\mathrm{Na}_{2} \mathrm{O}$ & 4.92 & 5.81 & 4.94 & 5.85 & 12.89 & 13.32 & 0.99 & 5.09 & 6.79 & 0.87 & 0.95 & 2.22 & 2.42 \\
\hline $\mathrm{K}_{2} \mathrm{O}$ & 0.03 & 0.01 & 0.01 & 0.02 & 0.00 & 0.02 & 0.04 & 0.03 & 0.02 & 0.01 & 0.01 & 0.00 & 0.00 \\
\hline $\mathrm{NiO}$ & 0.04 & 0.02 & 0.00 & 0.03 & 0.01 & 0.00 & 0.03 & 0.03 & 0.02 & 0.03 & 0.00 & 0.03 & 0.00 \\
\hline $\mathrm{Cr}_{2} \mathrm{O}_{3}$ & 0.05 & 0.04 & 0.05 & 0.04 & 0.03 & 0.05 & 0.01 & 0.13 & 0.09 & 0.01 & 0.00 & 0.04 & 0.07 \\
\hline $\mathrm{ZrO}_{2}$ & 0.31 & 0.32 & 0.45 & 0.23 & 0.00 & 0.20 & 0.06 & 0.15 & 0.11 & 0.01 & 0.03 & 0.10 & 0.14 \\
\hline Total & 98.56 & 99.37 & 99.37 & 98.60 & 96.60 & 96.58 & 99.74 & 99.46 & 99.50 & 99.58 & 99.22 & 98.79 & 99.33 \\
\hline $\mathrm{Fe}_{2} \mathrm{O}_{3}$ & 12.27 & 13.60 & 11.60 & 13.78 & 28.72 & 28.88 & 5.41 & 12.78 & 15.39 & 4.79 & 3.01 & 6.56 & 7.21 \\
\hline $\mathrm{FeO}$ & 1.87 & 2.18 & 2.96 & 1.92 & 0.95 & 0.00 & 0.52 & 1.31 & 0.72 & 0.28 & 2.30 & 2.84 & 2.63 \\
\hline Total* & 99.80 & 100.731 & 100.53 & 99.98 & 99.48 & 99.48 & 100.29 & 100.741 & 101.041 & 100.06 & 99.52 & 99.45 & 100.05 \\
\hline \multicolumn{14}{|c|}{ Element (ppm) } \\
\hline $\mathrm{Si}$ & 2.034 & 2.028 & 2.034 & 2.022 & 2.153 & 2.145 & 1.962 & 2.038 & 2.052 & 1.965 & 1.988 & 1.980 & 1.980 \\
\hline${ }^{\mathrm{IV}} \mathrm{Al}$ & 0.000 & 0.000 & 0.000 & 0.000 & 0.000 & 0.000 & 0.027 & 0.000 & 0.000 & 0.023 & 0.012 & 0.020 & 0.020 \\
\hline Sum & 2.034 & 2.028 & 2.034 & 2.022 & 2.153 & 2.145 & 1.989 & 2.038 & 2.052 & 1.988 & 2.000 & 2.000 & 2.000 \\
\hline${ }^{\mathrm{VI}} \mathrm{Al}$ & 0.028 & 0.054 & 0.038 & 0.048 & 0.043 & 0.024 & 0.000 & 0.014 & 0.043 & 0.000 & 0.007 & 0.035 & 0.034 \\
\hline $\mathrm{Ti}$ & 0.010 & 0.023 & 0.010 & 0.031 & 0.019 & 0.027 & 0.008 & 0.013 & 0.022 & 0.009 & 0.008 & 0.010 & 0.010 \\
\hline $\mathrm{Cr}$ & 0.001 & 0.001 & 0.002 & 0.001 & 0.001 & 0.002 & 0.000 & 0.004 & 0.003 & 0.000 & 0.000 & 0.001 & 0.002 \\
\hline $\mathrm{Fe}^{3+}$ & 0.357 & 0.397 & 0.335 & 0.403 & 0.891 & 0.953 & 0.151 & 0.367 & 0.444 & 0.134 & 0.085 & 0.188 & 0.205 \\
\hline $\mathrm{Mg}$ & 0.545 & 0.479 & 0.535 & 0.479 & 0.059 & 0.080 & 0.831 & 0.594 & 0.476 & 0.847 & 0.794 & 0.672 & 0.666 \\
\hline $\mathrm{Fe}^{2+}$ & 0.060 & 0.071 & 0.095 & 0.062 & 0.033 & 0.000 & 0.016 & 0.042 & 0.023 & 0.009 & 0.072 & 0.090 & 0.083 \\
\hline $\mathrm{Mn}$ & 0.007 & 0.009 & 0.010 & 0.007 & 0.013 & 0.010 & 0.010 & 0.005 & 0.006 & 0.008 & 0.006 & 0.011 & 0.010 \\
\hline $\mathrm{Ni}$ & 0.001 & 0.000 & 0.000 & 0.001 & 0.000 & 0.000 & 0.001 & 0.001 & 0.001 & 0.001 & 0.000 & 0.001 & 0.000 \\
\hline $\mathrm{Zr}$ & 0.006 & 0.006 & 0.008 & 0.004 & 0.000 & 0.004 & 0.001 & 0.003 & 0.002 & 0.000 & 0.001 & 0.002 & 0.003 \\
\hline $\mathrm{Ca}$ & 0.706 & 0.632 & 0.681 & 0.640 & 0.078 & 0.091 & 0.972 & 0.669 & 0.578 & 0.987 & 0.988 & 0.890 & 0.878 \\
\hline $\mathrm{Na}$ & 0.369 & 0.437 & 0.367 & 0.440 & 1.030 & 1.065 & 0.072 & 0.376 & 0.504 & 0.063 & 0.069 & 0.164 & 0.178 \\
\hline Sum & 2.089 & 2.109 & 2.081 & 2.116 & 2.168 & 2.256 & 2.062 & 2.088 & 2.102 & 2.057 & 2.028 & 2.064 & 2.070 \\
\hline$\%$ Wo & 42.17 & 39.81 & 41.12 & 40.27 & 7.28 & 8.05 & 49.09 & 39.91 & 37.85 & 49.74 & 50.82 & 48.08 & 47.66 \\
\hline$\%$ En & 32.52 & 30.15 & 32.31 & 30.09 & 5.52 & 7.03 & 41.95 & 35.41 & 31.20 & 42.67 & 40.85 & 36.29 & 36.13 \\
\hline$\% \mathrm{Fs}$ & 25.30 & 30.04 & 26.57 & 29.65 & 87.21 & 84.92 & 8.96 & 24.68 & 30.95 & 7.59 & 8.34 & 15.63 & 16.21 \\
\hline $\begin{array}{l}\mathrm{Mg} / \\
\left(\mathrm{Mg}+\mathrm{Fe}^{2+}\right)\end{array}$ & 56.64 & 50.55 & 55.47 & 50.72 & 6.03 & 7.72 & 83.22 & 59.25 & 50.51 & 85.57 & 83.57 & 70.73 & 69.76 \\
\hline
\end{tabular}

Structural formulae were recalculated to $6 \mathrm{O}^{2-}$ and 4 cations, with $\mathrm{Fe}(\mathrm{II})$ and $\mathrm{Fe}(\mathrm{III})$ determined on the basis of charge balance.

* The total following appropriate recalculation of $\mathrm{Fe}(\mathrm{II})$ and $\mathrm{Fe}(\mathrm{III})$. 
TABLE 12. Selected compositions of mica-group minerals from the intrusive carbonatites and nephelinites.

\begin{tabular}{|c|c|c|c|c|c|c|c|c|c|c|c|c|}
\hline $\begin{array}{l}\text { Oxide } \\
\text { (wt.\%) }\end{array}$ & $\begin{array}{l}\text { SV- } \\
529 \\
\text { Core }\end{array}$ & $\begin{array}{l}\text { SV- } \\
529 \\
\text { Rim }\end{array}$ & $\begin{array}{c}\text { SV- } \\
529 \\
\text { Core }\end{array}$ & $\begin{array}{l}\text { SV- } \\
529 \\
\text { Rim }\end{array}$ & $\begin{array}{l}\text { SV- } \\
531 \\
\text { Core }\end{array}$ & $\begin{array}{l}\text { SV- } \\
531 \\
\text { Rim }\end{array}$ & $\begin{array}{c}\text { SV- } \\
531 \\
\text { Small } \\
\text { crystal } \\
\mathrm{Cb}\end{array}$ & $\begin{array}{c}\text { SV- } \\
536 \\
\text { Core }\end{array}$ & $\begin{array}{l}\text { SV- } \\
536 \\
\text { Rim }\end{array}$ & $\begin{array}{c}\text { SV- } \\
536 \\
\text { Small } \\
\text { crystal } \\
\mathrm{Cb}\end{array}$ & $\begin{array}{l}\text { SV- } \\
537 \\
\text { Core }\end{array}$ & $\begin{array}{l}\text { SV- } \\
537 \\
\text { Rim }\end{array}$ \\
\hline $\mathrm{SiO}_{2}$ & 37.42 & 37.44 & 38.26 & 37.94 & 38.32 & 39.38 & 39.41 & 39.19 & 37.21 & 38.72 & 37.89 & 37.51 \\
\hline $\mathrm{TiO}_{2}$ & 3.45 & 3.09 & 3.32 & 3.36 & 2.17 & 2.10 & 2.01 & 2.05 & 2.20 & 2.58 & 1.74 & 2.57 \\
\hline $\mathrm{Al}_{2} \mathrm{O}_{3}$ & 11.96 & 12.21 & 12.22 & 12.20 & 12.25 & 11.51 & 11.78 & 12.92 & 13.61 & 13.44 & 11.66 & 12.17 \\
\hline $\mathrm{FeO}$ & 15.84 & 16.22 & 15.58 & 14.35 & 12.24 & 11.27 & 11.35 & 6.50 & 7.73 & 8.19 & 13.01 & 13.19 \\
\hline $\mathrm{MnO}$ & 0.27 & 0.28 & 0.24 & 0.26 & 0.19 & 0.18 & 0.23 & 0.14 & 0.18 & 0.22 & 0.54 & 0.39 \\
\hline $\mathrm{MgO}$ & 15.30 & 15.15 & 15.90 & 15.77 & 19.95 & 20.94 & 20.15 & 21.86 & 21.51 & 21.83 & 19.15 & 18.20 \\
\hline $\mathrm{CaO}$ & 0.07 & 0.08 & 0.12 & 0.09 & 0.58 & 0.32 & 0.56 & 0.45 & 0.56 & 0.17 & 0.16 & 0.07 \\
\hline $\mathrm{Na}_{2} \mathrm{O}$ & 0.36 & 0.36 & 0.35 & 0.26 & 0.32 & 0.39 & 0.36 & 0.75 & 1.04 & 1.01 & 0.47 & 0.50 \\
\hline $\mathrm{K}_{2} \mathrm{O}$ & 9.52 & 9.63 & 9.03 & 9.67 & 6.53 & 7.88 & 7.40 & 7.57 & 7.32 & 8.88 & 9.46 & 9.49 \\
\hline $\mathrm{NiO}$ & 0.01 & 0.00 & 0.00 & 0.00 & 0.03 & 0.00 & 0.02 & 0.00 & 0.05 & 0.00 & 0.00 & 0.08 \\
\hline $\mathrm{Cr}_{2} \mathrm{O}_{3}$ & 0.05 & 0.05 & 0.03 & 0.03 & 0.09 & 0.07 & 0.05 & 0.02 & 0.04 & 0.03 & 0.03 & 0.09 \\
\hline F & 0.30 & 0.37 & 0.30 & 0.41 & 0.38 & 0.35 & 0.32 & 0.18 & 0.14 & 0.16 & 0.27 & 0.16 \\
\hline $\mathrm{F}=\mathrm{O}$ & -0.13 & -0.16 & -0.13 & -0.17 & -0.16 & -0.15 & -0.14 & -0.07 & -0.06 & -0.07 & -0.11 & -0.07 \\
\hline $\mathrm{Cl}$ & 0.02 & 0.00 & 0.00 & 0.03 & 0.00 & 0.01 & 0.01 & 0.01 & 0.02 & 0.01 & 0.00 & 0.01 \\
\hline $\mathrm{Cl}=\mathrm{O}$ & -0.01 & -0.00 & -0.00 & -0.01 & -0.00 & -0.01 & -0.00 & -0.00 & -0.01 & -0.00 & -0.00 & -0.00 \\
\hline $\mathrm{SO}_{3}$ & 0.05 & 0.02 & 0.08 & 0.03 & 0.02 & 0.00 & 0.08 & 0.00 & 0.03 & 0.05 & 0.00 & 0.03 \\
\hline Total & 94.49 & 94.74 & 95.30 & 94.22 & 92.91 & 94.24 & 93.59 & 91.57 & 91.57 & 95.22 & 94.27 & 94.39 \\
\hline \multicolumn{13}{|c|}{ Element (ppm) } \\
\hline $\mathrm{Si}$ & 5.716 & 5.715 & 5.754 & 5.765 & 5.738 & 5.819 & 5.853 & 5.806 & 5.575 & 5.622 & 5.722 & 5.665 \\
\hline $\mathrm{Al}^{\mathrm{IV}}$ & 2.154 & 2.196 & 2.166 & 2.185 & 2.162 & 2.005 & 2.063 & 2.194 & 2.404 & 2.301 & 2.077 & 2.168 \\
\hline Sum & 7.870 & 7.912 & 7.919 & 7.950 & 7.900 & 7.824 & 7.916 & 8.000 & 7.979 & 7.923 & 7.799 & 7.832 \\
\hline${ }^{\mathrm{VI}} \mathrm{Al}$ & 0.000 & 0.000 & 0.000 & 0.000 & 0.000 & 0.000 & 0.000 & 0.063 & 0.000 & 0.000 & 0.000 & 0.000 \\
\hline $\mathrm{Ti}$ & 0.397 & 0.355 & 0.375 & 0.384 & 0.245 & 0.233 & 0.225 & 0.228 & 0.247 & 0.281 & 0.197 & 0.291 \\
\hline $\mathrm{Cr}$ & 0.006 & 0.005 & 0.003 & 0.004 & 0.010 & 0.008 & 0.006 & 0.003 & 0.005 & 0.003 & 0.004 & 0.011 \\
\hline $\mathrm{Mg}$ & 3.483 & 3.447 & 3.563 & 3.570 & 4.453 & 4.611 & 4.459 & 4.825 & 4.804 & 4.725 & 4.310 & 4.097 \\
\hline $\mathrm{Fe}^{2+}$ & 2.024 & 2.070 & 1.959 & 1.824 & 1.533 & 1.392 & 1.409 & 0.805 & 0.969 & 0.994 & 1.644 & 1.666 \\
\hline $\mathrm{Mn}$ & 0.035 & 0.036 & 0.031 & 0.034 & 0.024 & 0.023 & 0.029 & 0.017 & 0.022 & 0.027 & 0.069 & 0.050 \\
\hline $\mathrm{Ni}$ & 0.001 & 0.000 & 0.000 & 0.000 & 0.004 & 0.000 & 0.002 & 0.000 & 0.006 & 0.000 & 0.000 & 0.010 \\
\hline Sum & 5.946 & 5.913 & 5.931 & 5.817 & 6.268 & 6.267 & 6.130 & 5.942 & 6.053 & 6.030 & 6.223 & 6.125 \\
\hline $\mathrm{Ca}$ & 0.011 & 0.013 & 0.019 & 0.014 & 0.093 & 0.051 & 0.089 & 0.071 & 0.089 & 0.026 & 0.026 & 0.011 \\
\hline $\mathrm{Na}$ & 0.106 & 0.107 & 0.103 & 0.076 & 0.092 & 0.113 & 0.103 & 0.215 & 0.301 & 0.285 & 0.139 & 0.146 \\
\hline K & 1.855 & 1.875 & 1.733 & 1.875 & 1.247 & 1.486 & 1.403 & 1.431 & 1.400 & 1.645 & 1.822 & 1.828 \\
\hline Sum & 1.972 & 1.995 & 1.855 & 1.965 & 1.432 & 1.649 & 1.594 & 1.717 & 1.790 & 1.955 & 1.998 & 1.985 \\
\hline Total cations & 15.788 & 15.820 & 15.705 & 15.732 & 15.601 & 15.741 & 15.640 & 15.659 & 15.822 & 15.909 & 16.020 & 15.942 \\
\hline $\begin{array}{l}\mathrm{Mg} / \\
\left(\mathrm{Mg}+\mathrm{Fe}^{2+}\right)\end{array}$ & 0.632 & 0.625 & 0.645 & 0.662 & 0.744 & 0.768 & 0.760 & 0.857 & 0.832 & 0.826 & 0.724 & 0.711 \\
\hline
\end{tabular}

Structural formulae were calculated based on $22 \mathrm{O}^{2-}(\mathrm{OH}, \mathrm{F}, \mathrm{Cl})$ with all iron as $\mathrm{Fe}$ (II).

Abbreviations are: $\mathrm{Cb}=$ Carbonatite; Neph $=$ Nephelinite.

constant. The clinopyroxene in the amphibolemica-bearing nephelinite has a slight trend towards aegirine-augite compositions (squares in
Fig. 9a). The clinopyroxene in the rhönite-bearing nephelinite has a regular concentric chemical zonation, with a relatively large core and a 
TABLE 13. Selected analyses of iron and iron-titanium oxide minerals in the intrusive carbonatites and nephelinites.

\begin{tabular}{|c|c|c|c|c|c|c|c|c|}
\hline $\begin{array}{l}\text { Oxide } \\
\text { wt. } \%\end{array}$ & $\begin{array}{l}\text { SV-521 } \\
\text { Mt-Hm } \\
\text { Cb }\end{array}$ & $\begin{array}{l}\text { SV-521 } \\
\text { Mt-Hm } \\
\text { Cb }\end{array}$ & $\begin{array}{l}\text { SV-529 } \\
\text { Mt-Hm } \\
\text { Cb }\end{array}$ & $\begin{array}{l}\text { SV-529 } \\
\text { Mt-Hm } \\
\text { Cb }\end{array}$ & $\begin{array}{l}\text { SV-531 } \\
\text { Mt-Hm } \\
\text { Cb }\end{array}$ & $\begin{array}{c}\text { SV-537 } \\
\text { Mt } \\
\text { Neph }\end{array}$ & $\begin{array}{l}\text { SV-537 } \\
\text { Exsol } \\
\text { Neph }\end{array}$ & $\begin{array}{c}\text { SV-537 } \\
\text { Exsol } \\
\text { Neph }\end{array}$ \\
\hline $\mathrm{SiO}_{2}$ & 1.85 & 4.91 & 4.61 & 4.56 & 3.77 & 4.97 & 0.92 & 2.42 \\
\hline $\mathrm{TiO}_{2}$ & 0.00 & 0.00 & 0.03 & 0.04 & 0.01 & 1.17 & 17.06 & 23.53 \\
\hline $\mathrm{Al}_{2} \mathrm{O}_{3}$ & 0.00 & 0.00 & 0.11 & 0.17 & 1.89 & 2.59 & 1.90 & 1.98 \\
\hline $\mathrm{FeO}$ & 78.73 & 72.99 & 71.32 & 73.21 & 75.31 & 83.06 & 71.89 & 62.59 \\
\hline $\mathrm{MnO}$ & 0.18 & 0.71 & 0.07 & 0.23 & 0.48 & 0.00 & 0.19 & 0.09 \\
\hline $\mathrm{MgO}$ & 0.84 & 0.95 & 0.96 & 0.86 & 0.61 & 0.54 & 0.19 & 0.24 \\
\hline $\mathrm{CaO}$ & 0.65 & 0.46 & 0.49 & 0.86 & 0.47 & 0.20 & 0.35 & 0.40 \\
\hline $\mathrm{NiO}$ & 0.00 & 0.00 & 0.12 & 0.00 & 0.22 & 0.04 & 0.03 & 0.05 \\
\hline $\mathrm{Cr}_{2} \mathrm{O}_{3}$ & 0.00 & 0.00 & 0.06 & 0.02 & 0.02 & 0.07 & 0.07 & 0.05 \\
\hline Total & 82.24 & 80.03 & 77.77 & 79.95 & 82.78 & 92.79 & 92.60 & 91.34 \\
\hline $\mathrm{Fe}_{2} \mathrm{O}_{3}$ & 56.91 & 47.61 & 46.40 & 48.14 & 49.88 & 50.80 & 28.63 & 10.47 \\
\hline $\mathrm{FeO}$ & 27.52 & 30.15 & 29.56 & 29.90 & 30.42 & 37.35 & 46.12 & 53.17 \\
\hline Total* & 87.94 & 84.97 & 82.61 & 84.94 & 88.14 & 98.45 & 95.79 & 92.85 \\
\hline \multicolumn{9}{|c|}{ Element (ppm) } \\
\hline $\mathrm{Si}$ & 0.079 & 1.719 & 1.657 & 1.594 & 1.268 & 1.492 & 0.284 & 0.761 \\
\hline $\mathrm{Ti}$ & 0.000 & 0.000 & 0.009 & 0.009 & 0.002 & 0.265 & 3.981 & 5.575 \\
\hline $\mathrm{Al}$ & 0.000 & 0.000 & 0.047 & 0.070 & 0.749 & 0.918 & 0.695 & 0.735 \\
\hline $\mathrm{Fe}^{3+}$ & 1.841 & 12.544 & 12.544 & 12.667 & 12.627 & 11.478 & 6.685 & 2.482 \\
\hline $\mathrm{Fe}^{2+}$ & 0.990 & 8.828 & 8.881 & 8.743 & 8.558 & 9.378 & 11.968 & 14.007 \\
\hline $\mathrm{Mn}$ & 0.007 & 0.211 & 0.020 & 0.067 & 0.137 & 0.000 & 0.050 & 0.023 \\
\hline $\mathrm{Mg}$ & 0.054 & 0.498 & 0.511 & 0.448 & 0.305 & 0.159 & 0.086 & 0.111 \\
\hline $\mathrm{Ca}$ & 0.030 & 0.174 & 0.189 & 0.321 & 0.170 & 0.174 & 0.117 & 0.136 \\
\hline $\mathrm{Ni}$ & 0.000 & 0.000 & 0.034 & 0.000 & 0.060 & 0.008 & 0.007 & 0.012 \\
\hline $\mathrm{Cr}$ & 0.000 & 0.000 & 0.017 & 0.007 & 0.005 & 0.016 & 0.017 & 0.013 \\
\hline Sum & 23.981 & 23.974 & 23.909 & 23.926 & 23.881 & 23.888 & 23.890 & 23.855 \\
\hline
\end{tabular}

Abbreviations are: $\mathrm{Mt}-\mathrm{Hm}=$ magnetite - hematite; $\mathrm{Mt}=$ magnetite; Exsol = exsolution in magnetite; $\mathrm{Cb}=$ carbonatite; $\mathrm{Neph}=$ nephelinite.

Structural formulae were calculated on the basis of $32 \mathrm{O}^{2-}$ for magnetite; $36 \mathrm{O}^{2-}$ for ilmenite.

*The total following appropriate recalculation of Fe(II) and Fe(III).

subhedral rim (Fig. 9c). There is an increase in titanium and iron and a decrease in the magnesium content from core to rim. The sodium content remains constant but the aluminium content increases dramatically (up to 10 wt. $\%$, Table 15). These features are reflected in a different trend in Fig. $9 c$, which may indicate different changes in magma composition due to different crystallization conditions or volatile contents.

Hydrous minerals in the São Vicente nephelinites include amphibole and mica. The amphibole is kaersutite (5-6 wt. $\% \mathrm{TiO}_{2}$, Table 16), which is practically homogeneous. The mica has concentric zoning with an increase in titanium and aluminium, and a decrease in magnesium content from core to rim; the sodium and iron contents remain constant (Table 12). This pattern is very similar to the pattern of zonation in Ti-rich clinopyroxene in the rhönite-bearing nephelinites and it strongly suggests that the evolution of the magma was, to a certain extent, controlled by volatiles.

Iron-titanium oxides occur in the amphibolemica-bearing nephelinites as accessory anhedral minerals, as in the intrusive carbonatites. In the nephelinites, the minerals are better preserved, and this is reflected in the higher analytical totals 


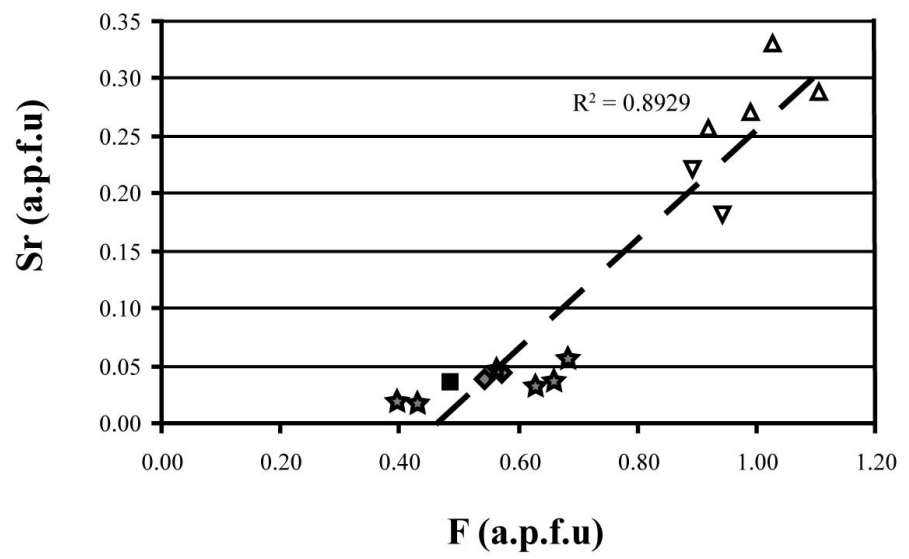

FIG. 10. Strontium vs. fluorine content in apatite from the São Vicente carbonatites and associated nephelinites. The symbols are: upward-facing triangles $=$ apatite from bands in extrusive carbonatites and dykes; downward-facing triangles $=$ apatite from band remnants in recrystallized extrusive carbonatites; diamonds $=$ isolated apatite crystals in recrystallized extrusive carbonatites; stars $=$ apatite from intrusive carbonatites; squares $=$ apatite from nephelinites.

in Table 13. The oxides are magnetite with thin Ti-rich exsolutions, containing $1-2 \mathrm{wt} . \% \mathrm{Al}_{2} \mathrm{O}_{3}$. An estimation of their crystallization temperature and oxygen fugacity was made using the ILMAT Excel worksheet (Lepage, 2003), with the geothermobarometric calibration of Andersen

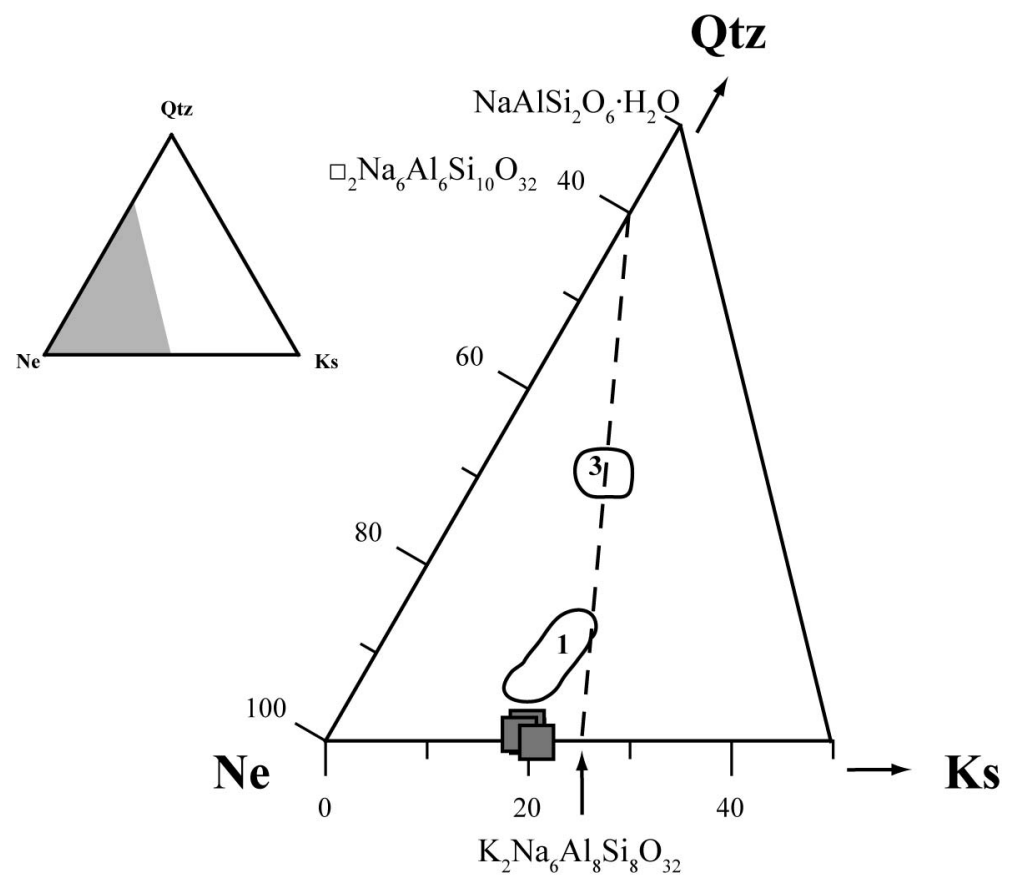

FIG. 11. A nepheline-kalsilite-quartz diagram for the São Vicente nephelinites. The dashed line corresponds to the compositional trend of nepheline co-crystallizing with $\mathrm{K}$-feldspar. The numbers 1 and 3 represent fields corresponding to early and late nepheline, respectively, and are after Henderson and Gibb (1983). 


$$
\text { C. DE IGNACIO ET AL. }
$$

TABLE 14. Representative analyses of nepheline phenocrysts from the hydrous nephelinites.

\begin{tabular}{|c|c|c|c|c|}
\hline Oxide (wt.\%) & SV-537 & SV-537 & SV-537 & SV -537 \\
\hline $\mathrm{SiO}_{2}$ & 41.11 & 41.91 & 41.39 & 40.87 \\
\hline $\mathrm{TiO}_{2}$ & 0.00 & 0.04 & 0.04 & 0.00 \\
\hline $\mathrm{Al}_{2} \mathrm{O}_{3}$ & 34.94 & 34.04 & 34.67 & 35.47 \\
\hline $\mathrm{FeO}$ & 0.46 & 0.46 & 0.60 & 0.49 \\
\hline $\mathrm{MnO}$ & 0.01 & 0.04 & 0.04 & 0.00 \\
\hline $\mathrm{MgO}$ & 0.00 & 0.01 & 0.03 & 0.04 \\
\hline $\mathrm{CaO}$ & 1.26 & 1.14 & 1.20 & 0.76 \\
\hline $\mathrm{Na}_{2} \mathrm{O}$ & 15.95 & 15.92 & 16.17 & 16.24 \\
\hline $\mathrm{K}_{2} \mathrm{O}$ & 6.22 & 6.04 & 5.94 & 6.66 \\
\hline $\mathrm{BaO}$ & 0.00 & 0.01 & 0.00 & 0.00 \\
\hline $\mathrm{SrO}$ & 0.00 & 0.00 & 0.00 & 0.00 \\
\hline Total & 99.94 & 100.60 & 100.08 & 100.52 \\
\hline Total $\mathrm{Fe}$ as & 0.51 & 0.51 & 0.67 & 0.54 \\
\hline $\mathrm{Fe}_{2} \mathrm{O}_{3}$ & & & & \\
\hline Total* & 99.99 & 100.65 & 100.15 & 100.58 \\
\hline \multicolumn{5}{|l|}{ Element (ppm) } \\
\hline $\mathrm{Si}$ & 3.982 & 4.020 & 4.002 & 3.946 \\
\hline $\mathrm{Al}$ & 3.990 & 3.962 & 3.952 & 4.036 \\
\hline $\mathrm{Ti}$ & 0.000 & 0.003 & 0.003 & 0.000 \\
\hline $\mathrm{Fe}^{3+}$ & 0.033 & 0.033 & 0.044 & 0.035 \\
\hline Sum & 8.006 & 8.019 & 8.001 & 8.017 \\
\hline $\mathrm{Mg}$ & 0.000 & 0.002 & 0.004 & 0.006 \\
\hline $\mathrm{Mn}$ & 0.001 & 0.003 & 0.003 & 0.000 \\
\hline $\mathrm{Ca}$ & 0.131 & 0.117 & 0.124 & 0.078 \\
\hline $\mathrm{Na}$ & 2.995 & 2.961 & 3.031 & 3.040 \\
\hline K & 0.768 & 0.739 & 0.732 & 0.820 \\
\hline $\mathrm{Ba}$ & 0.000 & 0.000 & 0.000 & 0.000 \\
\hline $\mathrm{Sr}$ & 0.000 & 0.000 & 0.000 & 0.000 \\
\hline Sum & 3.895 & 3.823 & 3.895 & 3.944 \\
\hline$\% \mathrm{Ne}$ & 79.67 & 79.23 & 80.38 & 79.14 \\
\hline$\% \mathrm{Ks}$ & 19.58 & 19.03 & 18.66 & 20.80 \\
\hline$\% Q t z$ & 0.75 & 1.74 & 0.97 & 0.06 \\
\hline
\end{tabular}

Each analysis is on a single crystal.

Structural formulae were calculated based on $16 \mathrm{O}^{2-}$ and 12 cations.

* This is the total with $\mathrm{Fe}(\mathrm{III})$.

Abbreviations are Ne, nepheline; Ka, kalsilite; Qtz, quartz.

and Lindsley (1985). The results are shown in Fig. 12. The Ti-rich exsolutions reflect progressive oxidation of magnetite as the temperature decreased from 825 to $752^{\circ} \mathrm{C}$, at oxygen fugacity conditions higher than the nickel-nickel oxide $(\mathrm{Ni}-\mathrm{NiO})$ buffer and near to the hematitemagnetite (H-M) buffer (Fig. 12). This relatively high oxygen fugacity is consistent with crystallization under volatile saturation conditions, in a relatively oxidizing environment.
The behaviour of titanium in rhönite-bearing nephelinites is controlled by the presence of clinopyroxene and rhönite, $\mathrm{Ca}_{2}\left(\mathrm{Mg}, \mathrm{Fe}^{2+}, \mathrm{Fe}^{3+}, \mathrm{Ti}\right)_{6}(\mathrm{Si}, \mathrm{Al})_{6} \mathrm{O}_{20}$. In the Areia Branca nephelinites, rhönite occurs as slender, euhedral prismatic crystals associated with clinopyroxene (Fig. $8 g$ ). It is opaque in planepolarized light. The compositions of rhönite from Areia Branca are listed in Table 17. They are similar to rhönite in tephrite-phonolite lavas 
TABLE 15. Representative compositions of clinopyroxene from the nephelinites.

\begin{tabular}{|c|c|c|c|c|c|c|c|c|c|c|}
\hline $\begin{array}{l}\text { Oxide } \\
\text { wt. } \%\end{array}$ & $\begin{array}{c}\text { SV-537 } \\
\text { Core }\end{array}$ & $\begin{array}{l}\text { SV-537 } \\
\text { Rim }\end{array}$ & $\begin{array}{c}\text { SV-537 } \\
\text { Core }\end{array}$ & $\begin{array}{l}\text { SV-537 } \\
\text { Rim }\end{array}$ & $\begin{array}{l}\text { SV-529 } \\
\text { Reaction } \\
\text { rim } \\
\text { (neph) }\end{array}$ & $\begin{array}{c}\text { SV-524 } \\
\text { Core }\end{array}$ & $\begin{array}{c}\text { SV-524 } \\
\rightarrow\end{array}$ & $\begin{array}{c}\mathrm{SV}-524 \\
\rightarrow\end{array}$ & $\begin{array}{c}\text { SV-524 } \\
\rightarrow\end{array}$ & $\begin{array}{c}\mathrm{SV}-524 \\
\text { Rim }\end{array}$ \\
\hline $\mathrm{SiO}_{2}$ & 43.26 & 42.53 & 43.33 & 41.93 & 49.87 & 44.81 & 45.32 & 42.50 & 41.93 & 42.82 \\
\hline $\mathrm{TiO}_{2}$ & 3.49 & 4.10 & 3.06 & 3.85 & 0.90 & 3.78 & 3.69 & 4.45 & 4.72 & 4.84 \\
\hline $\mathrm{Al}_{2} \mathrm{O}_{3}$ & 9.47 & 8.40 & 9.39 & 9.36 & 2.31 & 7.50 & 7.68 & 10.27 & 10.20 & 9.60 \\
\hline $\mathrm{FeO}$ & 6.87 & 8.68 & 6.94 & 8.93 & 7.14 & 6.13 & 6.47 & 7.18 & 7.09 & 7.25 \\
\hline $\mathrm{MnO}$ & 0.06 & 0.16 & 0.17 & 0.36 & 0.59 & 0.10 & 0.05 & 0.06 & 0.06 & 0.06 \\
\hline $\mathrm{MgO}$ & 11.71 & 10.39 & 11.42 & 10.00 & 13.84 & 12.32 & 12.36 & 11.07 & 10.93 & 10.76 \\
\hline $\mathrm{CaO}$ & 23.53 & 23.50 & 23.81 & 22.92 & 22.29 & 24.40 & 24.04 & 24.32 & 24.37 & 24.18 \\
\hline $\mathrm{Na}_{2} \mathrm{O}$ & 0.74 & 0.97 & 0.68 & 0.97 & 0.73 & 0.45 & 0.47 & 0.49 & 0.50 & 0.50 \\
\hline $\mathrm{K}_{2} \mathrm{O}$ & 0.00 & 0.00 & 0.02 & 0.00 & 1.01 & 0.00 & 0.02 & 0.00 & 0.01 & 0.02 \\
\hline $\mathrm{NiO}$ & 0.00 & 0.00 & 0.00 & 0.00 & 0.01 & 0.00 & 0.02 & 0.00 & 0.00 & 0.05 \\
\hline $\mathrm{Cr}_{2} \mathrm{O}_{3}$ & 0.05 & 0.03 & 0.03 & 0.04 & 0.05 & 0.11 & 0.00 & 0.12 & 0.02 & 0.01 \\
\hline $\mathrm{ZrO}_{2}$ & 0.03 & 0.03 & 0.00 & 0.11 & 0.00 & 0.03 & 0.00 & 0.02 & 0.00 & 0.06 \\
\hline Total & 99.20 & 98.77 & 98.86 & 98.49 & 98.70 & 99.63 & 100.12 & 100.47 & 99.82 & 100.14 \\
\hline $\mathrm{Fe}_{2} \mathrm{O}_{3}$ & 7.11 & 8.08 & 7.14 & 8.04 & 2.61 & 4.96 & 4.45 & 6.04 & 6.34 & 4.55 \\
\hline $\mathrm{FeO}$ & 0.47 & 1.41 & 0.52 & 1.70 & 4.79 & 1.67 & 2.46 & 1.74 & 1.38 & 3.16 \\
\hline Total* & 99.91 & 99.58 & 99.58 & 99.30 & 98.96 & 100.13 & 100.56 & 101.07 & 100.45 & 100.60 \\
\hline \multicolumn{11}{|l|}{ Element (ppm) } \\
\hline $\mathrm{Si}$ & 1.645 & 1.645 & 1.655 & 1.628 & 1.899 & 1.692 & 1.701 & 1.603 & 1.593 & 1.621 \\
\hline${ }^{\mathrm{IV}} \mathrm{Al}$ & 0.355 & 0.355 & 0.345 & 0.372 & 0.101 & 0.308 & 0.299 & 0.397 & 0.407 & 0.379 \\
\hline Sum & 2.000 & 2.000 & 2.000 & 2.000 & 2.000 & 2.000 & 2.000 & 2.000 & 2.000 & 2.000 \\
\hline${ }^{\mathrm{VI}} \mathrm{Al}$ & 0.070 & 0.028 & 0.078 & 0.056 & 0.003 & 0.026 & 0.040 & 0.059 & 0.050 & 0.049 \\
\hline $\mathrm{Ti}$ & 0.100 & 0.119 & 0.088 & 0.113 & 0.026 & 0.107 & 0.104 & 0.126 & 0.135 & 0.138 \\
\hline $\mathrm{Cr}$ & 0.002 & 0.001 & 0.001 & 0.001 & 0.001 & 0.003 & 0.000 & 0.003 & 0.001 & 0.000 \\
\hline $\mathrm{Fe}^{3+}$ & 0.204 & 0.235 & 0.205 & 0.235 & 0.075 & 0.141 & 0.126 & 0.171 & 0.181 & 0.130 \\
\hline $\mathrm{Mg}$ & 0.664 & 0.599 & 0.650 & 0.579 & 0.785 & 0.694 & 0.692 & 0.622 & 0.619 & 0.607 \\
\hline $\mathrm{Fe}^{2+}$ & 0.015 & 0.046 & 0.017 & 0.055 & 0.153 & 0.053 & 0.077 & 0.055 & 0.044 & 0.100 \\
\hline $\mathrm{Mn}$ & 0.002 & 0.005 & 0.006 & 0.012 & 0.019 & 0.003 & 0.001 & 0.002 & 0.002 & 0.002 \\
\hline $\mathrm{Ni}$ & 0.000 & 0.000 & 0.000 & 0.000 & 0.000 & 0.000 & 0.001 & 0.000 & 0.000 & 0.002 \\
\hline $\mathrm{Zr}$ & 0.000 & 0.001 & 0.000 & 0.002 & 0.000 & 0.001 & 0.000 & 0.000 & 0.000 & 0.001 \\
\hline $\mathrm{Ca}$ & 0.959 & 0.974 & 0.975 & 0.954 & 0.910 & 0.987 & 0.966 & 0.983 & 0.992 & 0.980 \\
\hline $\mathrm{Na}$ & 0.055 & 0.073 & 0.050 & 0.073 & 0.054 & 0.033 & 0.034 & 0.036 & 0.037 & 0.036 \\
\hline Sum & 2.069 & 2.080 & 2.070 & 2.080 & 2.025 & 2.047 & 2.042 & 2.058 & 2.061 & 2.044 \\
\hline$\% \mathrm{Wo}_{\mathrm{o}}$ & 52.03 & 52.40 & 52.62 & 51.99 & 46.86 & 52.57 & 51.89 & 53.60 & 53.97 & 53.90 \\
\hline$\%$ En & 36.02 & 32.23 & 35.10 & 31.56 & 40.46 & 36.95 & 37.14 & 33.95 & 33.68 & 33.38 \\
\hline$\% \mathrm{Fs}$ & 11.95 & 15.38 & 12.28 & 16.45 & 12.69 & 10.48 & 10.98 & 12.45 & 12.35 & 12.72 \\
\hline $\begin{array}{l}\mathrm{Mg} / \\
\left(\mathrm{Mg}+\mathrm{Fe}^{2+}\right)\end{array}$ & 75.24 & 68.10 & 74.56 & 66.62 & 77.55 & 78.20 & 77.31 & 73.33 & 73.32 & 72.57 \\
\hline
\end{tabular}

Core $\rightarrow$ Rim indicates a compositional profile.

Structural formulae were recalculated to $6 \mathrm{O}^{2-}$ and 4 cations, with $\mathrm{Fe}(\mathrm{II})$ and $\mathrm{Fe}(\mathrm{III})$ determined on the basis of charge balance.

* The total following appropriate recalculation of $\mathrm{Fe}(\mathrm{II})$ and $\mathrm{Fe}(\mathrm{III})$. 
TABLE 16. Selected compositions of amphibole from the nephelinites.

\begin{tabular}{|c|c|c|c|}
\hline $\begin{array}{l}\text { Oxide } \\
\text { (wt.\%) }\end{array}$ & $\begin{array}{l}\text { SV-537 } \\
\text { Core }\end{array}$ & $\begin{array}{c}\text { SV-537 } \\
\text { Rim }\end{array}$ & $\begin{array}{c}\text { SV-537 } \\
\text { Core }\end{array}$ \\
\hline $\mathrm{SiO}_{2}$ & 37.93 & 37.95 & 37.45 \\
\hline $\mathrm{TiO}_{2}$ & 5.20 & 5.15 & 6.04 \\
\hline $\mathrm{Al}_{2} \mathrm{O}_{3}$ & 15.09 & 14.95 & 14.24 \\
\hline $\mathrm{FeO}$ & 9.83 & 9.21 & 10.06 \\
\hline $\mathrm{MnO}$ & 0.24 & 0.25 & 0.10 \\
\hline $\mathrm{MgO}$ & 13.35 & 13.47 & 13.01 \\
\hline $\mathrm{CaO}$ & 12.41 & 12.66 & 12.57 \\
\hline $\mathrm{Na}_{2} \mathrm{O}$ & 2.55 & 2.46 & 2.44 \\
\hline $\mathrm{K}_{2} \mathrm{O}$ & 1.49 & 1.65 & 1.61 \\
\hline $\mathrm{NiO}$ & 0.05 & 0.00 & 0.00 \\
\hline $\mathrm{Cr}_{2} \mathrm{O}_{3}$ & 0.06 & 0.04 & 0.04 \\
\hline Total & 98.19 & 97.79 & 97.58 \\
\hline \multicolumn{4}{|c|}{ Element (ppm) } \\
\hline $\mathrm{Si}$ & 5.601 & 5.641 & 5.615 \\
\hline${ }^{\mathrm{IV}} \mathrm{Al}$ & 2.399 & 2.359 & 2.385 \\
\hline Sum & 8.000 & 8.000 & 8.000 \\
\hline${ }^{\mathrm{VI}} \mathrm{Al}$ & 0.228 & 0.260 & 0.132 \\
\hline $\mathrm{Ti}$ & 0.578 & 0.575 & 0.681 \\
\hline $\mathrm{Cr}$ & 0.007 & 0.005 & 0.005 \\
\hline $\mathrm{Fe}^{3+}$ & 0.070 & 0.000 & 0.000 \\
\hline $\mathrm{Mg}$ & 2.938 & 2.984 & 2.907 \\
\hline $\mathrm{Fe}^{2+}$ & 1.144 & 1.144 & 1.262 \\
\hline $\mathrm{Mn}$ & 0.030 & 0.031 & 0.013 \\
\hline $\mathrm{Ni}$ & 0.005 & 0.000 & 0.000 \\
\hline Sum & 5.000 & 5.000 & 5.000 \\
\hline $\mathrm{Ca}$ & 1.964 & 2.000 & 2.000 \\
\hline $\mathrm{Na}$ & 0.036 & 0.000 & 0.000 \\
\hline Sum & 2.000 & 2.000 & 2.000 \\
\hline $\mathrm{Ca}$ & 0.000 & 0.016 & 0.019 \\
\hline $\mathrm{Na}$ & 0.693 & 0.710 & 0.710 \\
\hline K & 0.281 & 0.312 & 0.308 \\
\hline Sum & 0.975 & 1.039 & 1.038 \\
\hline Total cations & 15.975 & 16.039 & 16.038 \\
\hline $\begin{array}{l}\mathrm{Mg} / \\
\left(\mathrm{Mg}+\mathrm{Fe}^{2+}\right)\end{array}$ & 0.720 & 0.723 & 0.697 \\
\hline
\end{tabular}

Structural formulae are calculated based on $23 \mathrm{O}^{2-}$, using the $13-\mathrm{CNK}$ normalization procedure for calcic amphiboles.

from other localities in the Cape Verde Islands (Fogo and Brava; Kogarko et al., 2005) and to rhönite in nephelinites from Iceland (Prestvik et al., 1999) (Table 17). In many cases, rhönite is considered to be an alteration product of primary amphibole (Ulrych et al., 2006), but this does not seem to be the case in the Areia Branca nephelinites, where the textural and compositional evidence suggests that rhönite is a primary phase. Prestvik et al. (1999) noted that rhönite formation requires very low pressures $(<60 \mathrm{MPa}$, which corresponds to depths shallower than $\sim 2 \mathrm{~km}$ ), high temperature $\left(840-1200^{\circ} \mathrm{C}\right)$ and the presence of a fluid phase. In São Vicente, where both types of nephelinite are similar in bulk composition, we suggest that the rhönite-bearing nephelinites crystallized at higher temperatures, whereas the amphibole-mica-bearing nephelinites crystallized at higher volatile pressures, lower temperatures and higher oxygen fugacities, as shown by $\mathrm{Fe}-\mathrm{Ti}$ oxide geothermobarometry. We were unable to estimate the oxygen fugacity in the rhönitebearing nephelinites, as magnetite and ilmenite are absent; Kogarko et al. (2005) calculated that rhönite phenocrysts in tephrite-phonolite lavas from Fogo and Brava formed close to the quartzfayalite-magnetite (QFM) buffer, at temperatures between 1150 and $1050^{\circ} \mathrm{C}$. The conditions for rhönite crystallization in the São Vicente nephelinites are probably similar or slightly reduced, considering the absence of magnetite, which is consistent with our interpretation.

\section{Whole-rock geochemistry}

Geochemical analyses of the carbonatites and nephelinites are presented in Table 18. All of the São Vicente intrusive and recrystallized carbonatites are calciocarbonatites in the classification of Woolley and Kempe (1989) (Fig. 13). The extrusive carbonatite sample (SV-499) has a substantial magnesium content (10.5 wt.\% $\mathrm{MgO}$, Table 18), and is a magnesiocarbonatite, and the dyke sample (SV-538), with 7.0 wt.\% MgO lies on the boundary between calciocarbonatites and magnesiocarbonatites (Table 18; Fig. 13). The high magnesium contents are the result of abundant dolomite bands. The recrystallized extrusive carbonatites only contain remnant bands and have lower magnesium contents (4.9-1.3 wt.\% MgO, Table 18). Among the intrusive carbonatites there are samples (SV-535, SV-536) with similar magnesium contents to the recrystallized samples (6.3-5.6 wt.\% MgO, Table 18), but the magnesium is in clinopyroxene, which is compositionally similar to the clinopyroxene in the nephelinites. The nephelinites have magnesium contents of $6.8-4.6$ wt.\% $\mathrm{MgO}$ (Table 18). 


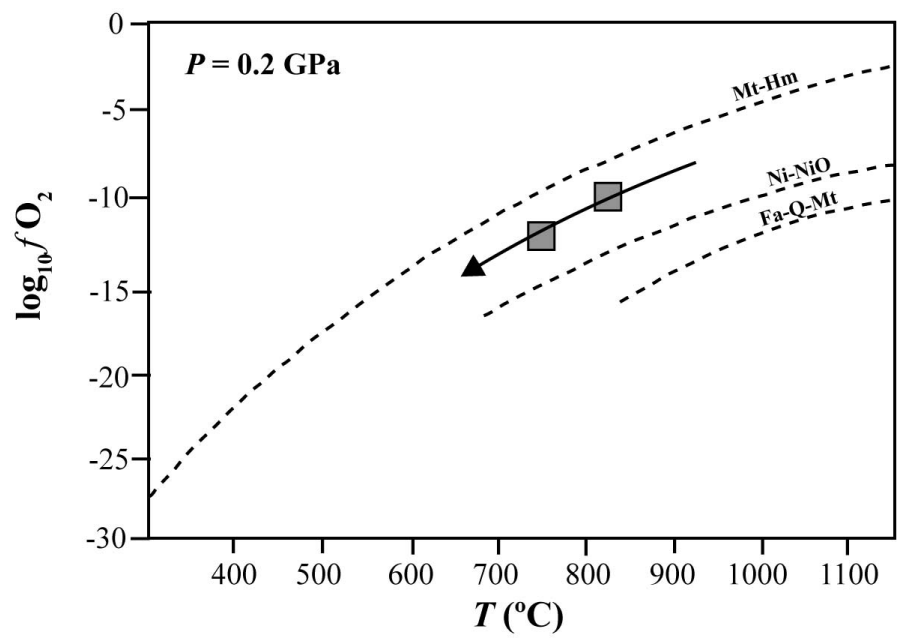

Fig. 12. A diagram showing oxygen fugacity conditions as a function of temperature for Fe-Ti oxides in the nephelinites from São Vicente. The oxygen fugacity buffers are from Wones and Eugster (1965).

The phosphorus content in the carbonatites correlates with modal apatite concentrations. The maximum $\mathrm{P}_{2} \mathrm{O}_{5}$ contents are in the extrusive carbonatite (sample SV-499; 5.1 wt.\%) and one of the intrusive carbonatites (SV-529; 6.5 wt.\%, Table 18). Alkali concentrations are close to the detection limit in all the carbonatites, ranging from $0.01-0.45$ wt. $\% \mathrm{Na}_{2} \mathrm{O}$ and $0.01-0.64$ wt. $\%$ $\mathrm{K}_{2} \mathrm{O}$. The highest sodium concentrations are in the extrusive carbonatites, which contain Na-rich apatite. Apatite also controls the total REE concentrations, which are up to ten times higher

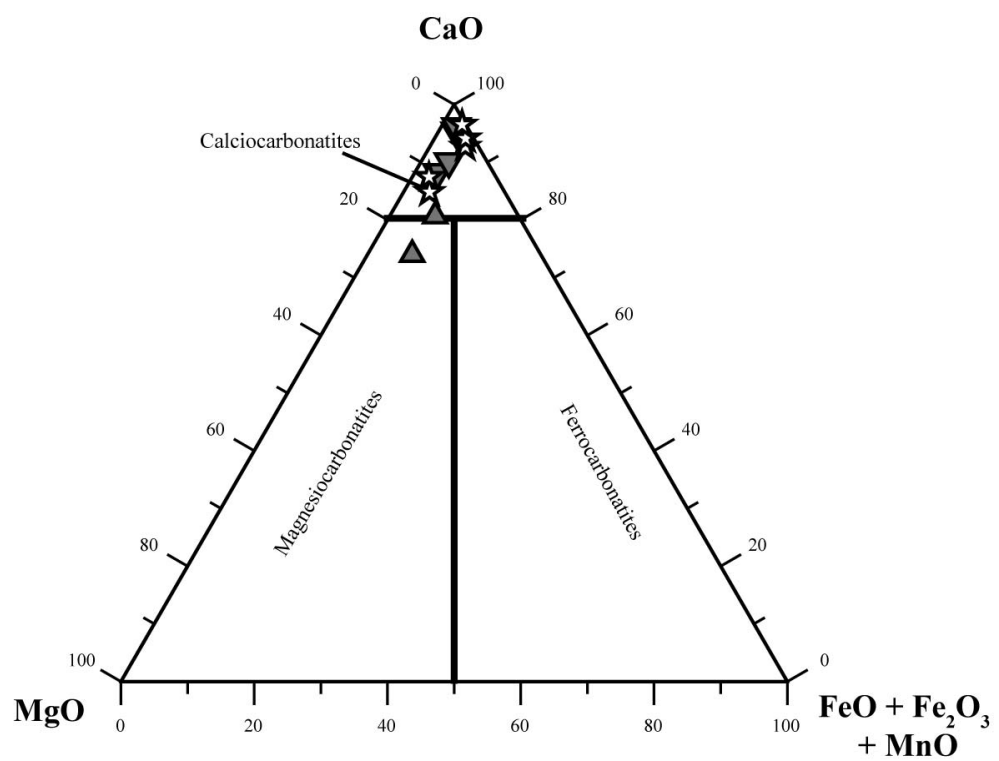

FIG. 13. A plot of the $\mathrm{CaO}-\mathrm{MgO}-\mathrm{FeO}$ whole-rock composition of the São Vicente carbonatites in the chemical classification of Woolley and Kempe (1989). The symbols are: upward-facing triangles = extrusive carbonatites and dykes; downward-facing triangles $=$ recrystallized carbonatites; stars $=$ intrusive carbonatites . 


$$
\text { C. DE IGNACIO ET AL. }
$$

TABLE 17. Selected rhönite analyses from the São Vicente nephelinites, with a comparison to Icelandic rhönite.

\begin{tabular}{|c|c|c|c|c|c|c|}
\hline $\begin{array}{l}\text { Oxide } \\
\text { wt. } \%\end{array}$ & $\begin{array}{c}\text { SV-537 } \\
\text { Core }\end{array}$ & $\begin{array}{c}\text { SV-537 } \\
\text { Rim }\end{array}$ & $\begin{array}{c}\text { SV-537 } \\
\text { Core }\end{array}$ & $\begin{array}{l}\text { SV-537 } \\
\text { Core }\end{array}$ & Iceland* & Iceland* \\
\hline $\mathrm{SiO}_{2}$ & 23.43 & 23.24 & 23.16 & 23.05 & 22.16 & 22.52 \\
\hline $\mathrm{TiO}_{2}$ & 11.72 & 11.61 & 11.76 & 10.86 & 8.69 & 8.41 \\
\hline $\mathrm{Al}_{2} \mathrm{O}_{3}$ & 18.02 & 17.96 & 18.21 & 17.71 & 18.98 & 19.08 \\
\hline $\mathrm{FeO}$ & 20.46 & 21.48 & 20.19 & 21.28 & 20.35 & 20.03 \\
\hline $\mathrm{MnO}$ & 0.11 & 0.03 & 0.07 & 0.11 & 0.16 & 0.12 \\
\hline $\mathrm{MgO}$ & 12.76 & 12.32 & 12.51 & 12.22 & 13.72 & 13.74 \\
\hline $\mathrm{CaO}$ & 12.69 & 12.84 & 12.81 & 12.81 & 13.35 & 12.95 \\
\hline $\mathrm{Na}_{2} \mathrm{O}$ & 0.78 & 0.84 & 0.87 & 0.79 & 0.40 & 0.44 \\
\hline $\mathrm{K}_{2} \mathrm{O}$ & 0.00 & 0.00 & 0.02 & 0.01 & n.a. & n.a. \\
\hline Total & 99.96 & 100.31 & 99.59 & 98.85 & 98.00 & 97.45 \\
\hline $\mathrm{Fe}_{2} \mathrm{O}_{3}$ & 7.34 & 8.35 & 7.56 & 8.98 & - & - \\
\hline $\mathrm{FeO}$ & 13.85 & 13.97 & 13.38 & 13.20 & - & - \\
\hline Total* & 100.70 & 101.15 & 100.35 & 99.75 & - & - \\
\hline \multicolumn{7}{|c|}{ Element (ppm) } \\
\hline $\mathrm{Si}$ & 3.190 & 3.170 & 3.165 & 3.193 & - & - \\
\hline${ }^{\mathrm{IV}} \mathrm{Al}$ & 2.810 & 2.830 & 2.835 & 2.807 & & \\
\hline Sum & 6.000 & 6.000 & 6.000 & 6.000 & - & - \\
\hline${ }^{\mathrm{VI}} \mathrm{Al}$ & 0.080 & 0.057 & 0.096 & 0.085 & - & - \\
\hline $\mathrm{Ti}$ & 1.200 & 1.191 & 1.208 & 1.132 & & \\
\hline $\mathrm{Fe}^{3+}$ & 0.752 & 0.857 & 0.777 & 0.936 & - & - \\
\hline $\mathrm{Mg}$ & 2.589 & 2.505 & 2.549 & 2.523 & & \\
\hline $\mathrm{Fe}^{2+}$ & 1.577 & 1.594 & 1.529 & 1.530 & - & - \\
\hline $\mathrm{Mn}$ & 0.013 & 0.003 & 0.008 & 0.013 & & \\
\hline Sum & 6.211 & 6.207 & 6.168 & 6.219 & - & - \\
\hline $\mathrm{Ca}$ & 1.851 & 1.877 & 1.875 & 1.902 & - & - \\
\hline $\mathrm{Na}$ & 0.206 & 0.222 & 0.232 & 0.213 & & \\
\hline K & 0.000 & 0.000 & 0.003 & 0.002 & - & - \\
\hline Sum & 2.058 & 2.099 & 2.109 & 2.116 & & \\
\hline
\end{tabular}

The Fe(II) and Fe(III) contents were calculated by charge balance.

Structural formulae are normalized to $20 \mathrm{O}^{2-}$.

* Rhönite compositions for Icelandic nephelinites taken from Prestvik et al. (1999); the abbreviation n.a. is not analysed.

in the extrusive carbonatites in comparison to the other carbonatites (Table 18).

The extrusive carbonatites (whether recrystallized or not) are significantly depleted in potassium, and this is reflected in the multielement diagram in Fig. 14 by a negative $\mathrm{K}$ anomaly. This anomaly is not as pronounced in the intrusive carbonatites, which contain biotitephlogopite micas; their potassium pattern is similar to that of the nephelinites (Fig. 14). The silica (4.5-0.6 wt.\%) and aluminium
(1.4-0.1 wt.\%) concentrations are higher in the intrusive carbonatites (Table 18), due to the presence of silicate minerals.

Trace elements which are compatible with direct melts from a peridotitic or wehrlitic mantle, such as chromium, nickel and cobalt, are present in low concentrations in all of the carbonatites (Table 18). They are less concentrated in the extrusive carbonatites, whether recrystallized or not $(\mathrm{Cr}=15-3 \mathrm{ppm} ; \mathrm{Ni}=$ $25-10 \mathrm{ppm}$; $\mathrm{Co}=7-3 \mathrm{ppm})$ than in the intrusive 
TABLE 18. Whole-rock geochemistry of carbonatites and nephelinites from São Vicente.

\begin{tabular}{lcccccccccccccc}
\hline Oxide & SV- & SV- & SV- & SV- & SV- & SV- & SV- & SV- & SV- & SV- & SV- & SV- & SV- & SV- \\
& 499 & 538 & 625 & 520 & 521 & 523 & 525 & 529 & 530 & 531 & 535 & 536 & 537 & 524 \\
& EC & Dyke & REC & REC & REC & REC & REC & IC & IC & IC & IC & IC & Neph & Neph \\
\hline
\end{tabular}

\begin{tabular}{|c|c|c|c|c|c|c|c|c|c|c|c|c|c|c|}
\hline \multicolumn{15}{|c|}{ Major elements (wt.\%) } \\
\hline $\mathrm{SiO}_{2}$ & 0.48 & 0.70 & 1.01 & 0.39 & 0.14 & 0.31 & 0.40 & 3.96 & 1.36 & 0.63 & 4.45 & 2.14 & 35.18 & 38.03 \\
\hline $\mathrm{TiO}_{2}$ & 0.04 & 0.09 & 0.02 & 0.05 & 0.03 & 0.12 & 0.08 & 0.22 & 0.08 & 0.03 & 0.27 & 0.12 & 3.89 & 4.08 \\
\hline $\mathrm{Al}_{2} \mathrm{O}_{3}$ & 0.05 & 0.14 & 0.05 & 0.06 & 0.04 & 0.07 & 0.04 & 0.88 & 0.37 & 0.12 & 1.41 & 0.69 & 11.91 & 10.62 \\
\hline $\mathrm{Fe}_{2} \mathrm{O}_{3 \mathrm{t}}$ & 2.40 & 3.00 & 1.40 & 1.13 & 1.32 & 2.03 & 1.10 & 2.63 & 2.41 & 1.46 & 1.96 & 1.20 & 13.39 & 11.36 \\
\hline $\mathrm{MnO}$ & 1.12 & 0.64 & 0.56 & 0.31 & 0.23 & 0.38 & 0.29 & 0.16 & 0.16 & 0.16 & 0.10 & 0.09 & 0.24 & 0.12 \\
\hline $\mathrm{MgO}$ & 10.48 & 6.97 & 4.88 & 1.57 & 1.53 & 3.53 & 1.27 & 1.25 & 0.90 & 0.52 & 6.28 & 5.58 & 6.75 & 4.63 \\
\hline $\mathrm{CaO}$ & 39.44 & 43.74 & 46.88 & 52.70 & 52.73 & 48.86 & 53.02 & 48.95 & 52.73 & 54.38 & 45.57 & 46.74 & 14.76 & 16.83 \\
\hline $\mathrm{Na}_{2} \mathrm{O}$ & 0.45 & 0.11 & 0.16 & 0.11 & 0.21 & 0.23 & 0.10 & 0.16 & 0.05 & 0.06 & 0.13 & 0.10 & 2.99 & 3.36 \\
\hline $\mathrm{K}_{2} \mathrm{O}$ & 0.07 & 0.03 & $<0.01$ & 0.07 & 0.02 & 0.05 & 0.08 & 0.64 & 0.13 & 0.03 & 0.45 & 0.19 & 1.19 & 1.13 \\
\hline $\mathrm{P}_{2} \mathrm{O}_{5}$ & 5.13 & 0.84 & 2.43 & 0.68 & 4.83 & 4.11 & 0.82 & 6.49 & 0.50 & 0.44 & 3.97 & 2.58 & 1.87 & 1.18 \\
\hline $\mathrm{CO}_{2}$ & 36.60 & 40.90 & 40.6 & 41.30 & 37.50 & 37.10 & 41.40 & 31.90 & 40.10 & 41.30 & 34.70 & 38.80 & 2.81 & 5.27 \\
\hline $\mathrm{LOI}^{*}$ & 1.41 & 0.57 & 0.00 & 0.71 & 0.41 & 1.21 & 0.60 & 0.82 & 1.09 & 0.72 & 1.42 & 0.82 & 3.87 & 4.21 \\
\hline Total & 97.66 & 97.73 & 97.74 & 99.06 & 99.00 & 97.98 & 99.19 & 98.05 & 99.89 & 99.84 & 100.70 & 99.05 & 98.85 & 100.80 \\
\hline $\begin{array}{l}\mathrm{Mg} / \\
\left(\mathrm{Mg}+\mathrm{Fe}^{2+}\right)\end{array}$ & 90.86 & 84.10 & 88.81 & 75.99 & 72.53 & 79.84 & 72.45 & 51.98 & 45.96 & 44.79 & 87.95 & 91.37 & 53.45 & 48.14 \\
\hline
\end{tabular}

Trace elements (ppm)

$\begin{array}{lcccccccccccccccc}\mathrm{Ba} & 7083 & 7070 & 2112 & 5056 & 2338 & 5529 & 3619 & 357 & 486 & 314 & 390 & 383 & 2165 & 514 \\ \mathrm{Co} & 3.2 & 3.9 & 7.4 & 2.1 & 2.5 & 3 & 1.6 & 9.1 & 13.9 & 4.4 & 6.1 & 5.3 & 38.7 & 39.3 \\ \mathrm{Cr} & 4.0 & 15.4 & 5.9 & 5.8 & 2.6 & 8.4 & 5.0 & 9.9 & 20.1 & 10.8 & 12.6 & 9.2 & 14.7 & 70.7 \\ \mathrm{Cs} & <0.05 & 0.09 & 0.09 & <0.05 & <0.05 & <0.05 & <0.05 & 0.51 & 0.2 & 0.06 & 1.83 & 0.79 & 0.47 & 1.27 \\ \mathrm{Hf} & 1.4 & 0.7 & 0.9 & 0.2 & 0.5 & 0.7 & 0.4 & 1.0 & 0.4 & 0.5 & 0.4 & 0.4 & 7.2 & 6.9 \\ \mathrm{Li} & 75.7 & 4.5 & <0.5 & 48.9 & 10.4 & 19.6 & 88.8 & 3.4 & 2.1 & <0.5 & 11.5 & 6.5 & 25.4 & 9.8 \\ \mathrm{Nb} & 161 & 177 & 18.1 & 56.2 & 29.1 & 114 & 93.5 & 10.3 & 11.4 & 7.8 & 21.7 & 11.0 & 103 & 59.4 \\ \mathrm{Ni} & 10.3 & 18.4 & 25.1 & 10.2 & 11.4 & 14.9 & 11.8 & 15.6 & 50.0 & 25.9 & 99.1 & 63.9 & 43.4 & 110 \\ \mathrm{~Pb} & 63.2 & 36.3 & 12.5 & 113 & 81.6 & 97.8 & 74.3 & 2.9 & 3.0 & 2.3 & 3.3 & 3.1 & 8.3 & 2.3 \\ \mathrm{Rb} & 1.9 & 1.5 & 0.5 & 2.4 & 0.5 & 10 . & 2.7 & 46.6 & 11.3 & 3.4 & 38.4 & 18.0 & 31.4 & 17.3 \\ \mathrm{Sc} & 11 & 8 & 2 & 4 & 6 & 10 & 5 & 3 & 4 & 4 & 2 & 2 & 19 & 28 \\ \mathrm{Sr} & 5306 & 2870 & 2871 & 3660 & 4840 & 4728 & 2897 & 5281 & 3795 & 3995 & 3032 & 3382 & 1014 & 951 \\ \mathrm{Ta} & 0.0 & 0.1 & 0.0 & 0.4 & 0.0 & 0.4 & 0.1 & 0.0 & 0.2 & 0.2 & 1.4 & 0.8 & 6.3 & 3.2 \\ \mathrm{Th} & 53.9 & 55.8 & 0.9 & 11.5 & 62.5 & 73.0 & 21.2 & 2.8 & 1.5 & 1.9 & 8.1 & 7.0 & 8.2 & 3.7 \\ \mathrm{U} & 3.3 & 0.7 & 3.5 & 0.9 & 6.0 & 5.8 & 3.8 & 0.8 & 1.4 & 1.6 & 1.1 & 0.7 & 0.9 & 10.2 \\ \mathrm{~V} & 292 & 176 & 374 & 95 & 102 & 191 & 100 & 148 & 69 & 50 & 42 & 26 & 372 & 380 \\ \mathrm{Y} & 322 & 186 & 96 & 55 & 131 & 167 & 128 & 69 & 45 & 46 & 59 & 62 & 39 & 23 \\ \mathrm{Zn} & 960 & 130 & 150 & 180 & 90 & 290 & 70 & 40 & 30 & 30 & 40 & 30 & 120 & 90 \\ \mathrm{Zr} & 106 & 43 & 188 & 15 & 65 & 78 & 27 & 173 & 43 & 56 & 45 & 31 & 399 & 397\end{array}$

Rare earth elements (ppm)

\begin{tabular}{|c|c|c|c|c|c|c|c|c|c|c|c|c|c|c|}
\hline $\mathrm{La}$ & 1030 & 540 & 376 & 108 & 92 & 215 & 152 & 170 & 116 & 124 & 181 & 200 & 98 & 72 \\
\hline $\mathrm{Ce}$ & 1660 & 1010 & 513 & 111 & 113 & 293 & 189 & 306 & 211 & 224 & 344 & 380 & 188 & 129 \\
\hline $\operatorname{Pr}$ & 171 & 114 & 49.3 & 9.87 & 11.9 & 29.7 & 17.8 & 33.6 & 23.1 & 25 & 39.4 & 42.3 & 23.1 & 14.8 \\
\hline $\mathrm{Nd}$ & 594 & 423 & 156 & 34 & 46 & 106 & 62.6 & 126 & 85.8 & 91.7 & 148 & 158 & 91.2 & 53.2 \\
\hline Sm & 86.9 & 68.9 & 23.9 & 7.1 & 10.4 & 20.4 & 17.1 & 21.6 & 14.6 & 15.8 & 25.6 & 26.5 & 16.8 & 9.4 \\
\hline $\mathrm{Eu}$ & 25.3 & 20.1 & 7.1 & 2.6 & 3.8 & 6.9 & 7.5 & 6.5 & 4.2 & 4.5 & 7.3 & 7.5 & 4.8 & 2.7 \\
\hline $\mathrm{Gd}$ & 69.7 & 52.3 & 20.2 & 8.6 & 12.3 & 21.2 & 26.8 & 17.9 & 11.9 & 12.4 & 19.6 & 20.2 & 13.1 & 7.5 \\
\hline $\mathrm{Tb}$ & 10.5 & 7.9 & 2.8 & 1.4 & 2.4 & 3.8 & 4.7 & 2.6 & 1.7 & 1.7 & 2.6 & 2.7 & 1.8 & 1.0 \\
\hline Dy & 61.3 & 40.5 & 15.0 & 8.6 & 17.0 & 24.2 & 25.9 & 13.5 & 8.8 & 9.2 & 12.7 & 13.3 & 8.8 & 5.0 \\
\hline Ho & 12.1 & 7.1 & 2.9 & 1.7 & 4.1 & 5.4 & 4.6 & 2.6 & 1.6 & 1.7 & 2.3 & 2.4 & 1.6 & 0.9 \\
\hline Er & 31.5 & 17.8 & 7.8 & 4.8 & 13.0 & 16.1 & 10.7 & 6.9 & 4.5 & 4.7 & 5.9 & 6.3 & 3.9 & 2.2 \\
\hline $\mathrm{Tm}$ & 4.1 & 2.3 & 1.0 & 0.7 & 2.1 & 2.5 & 1.3 & 0.9 & 0.6 & 0.6 & 0.8 & 0.8 & 0.5 & 0.3 \\
\hline $\mathrm{Yb}$ & 23.3 & 13.5 & 5.9 & 5.0 & 15.0 & 18.1 & 7.9 & 5.8 & 3.8 & 4.0 & 4.6 & 5.2 & 2.9 & 1.6 \\
\hline $\mathrm{Lu}$ & 3.2 & 2.0 & 0.8 & 0.8 & 3.0 & 3.6 & 1.3 & 0.9 & 0.6 & 0.7 & 0.8 & 0.8 & 0.4 & 0.2 \\
\hline$\Sigma R E E$ & 3783 & 2319 & 1182 & 304 & 346 & 766 & 529 & 715 & 488 & 520 & 794 & 866 & 455 & 300 \\
\hline
\end{tabular}

Abbreviations are: $\mathrm{EC}=$ extrusive carbonatite; $\mathrm{REC}=$ recrystallized, extrusive carbonatite;

$\mathrm{IC}=$ intrusive carbonatite; Neph $=$ nephelinite.

* LOI is the Loss on ignition other than $\mathrm{CO}_{2}$.

$\Sigma R E E$ is total rare earth element concentration in ppm. 
C. DE IGNACIO ET AL.

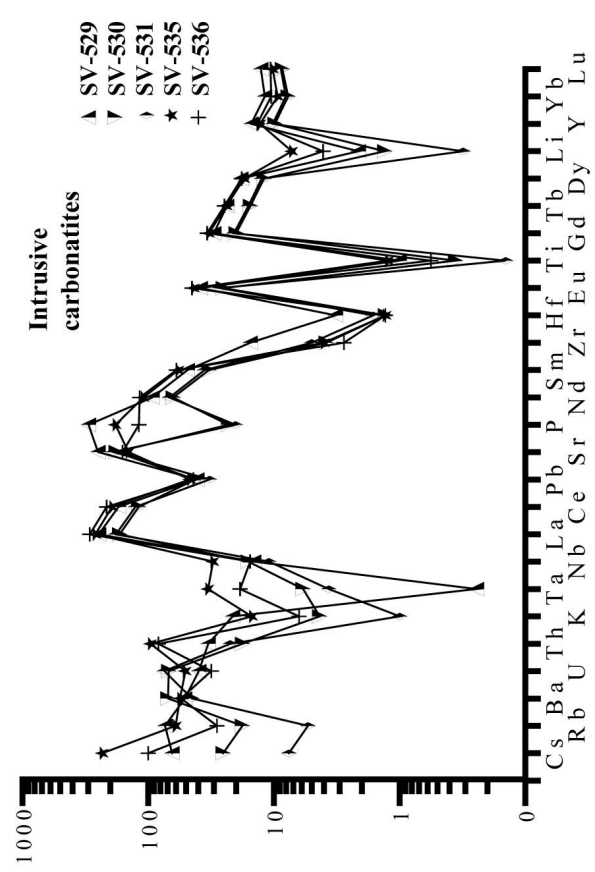

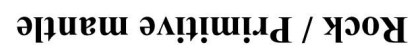

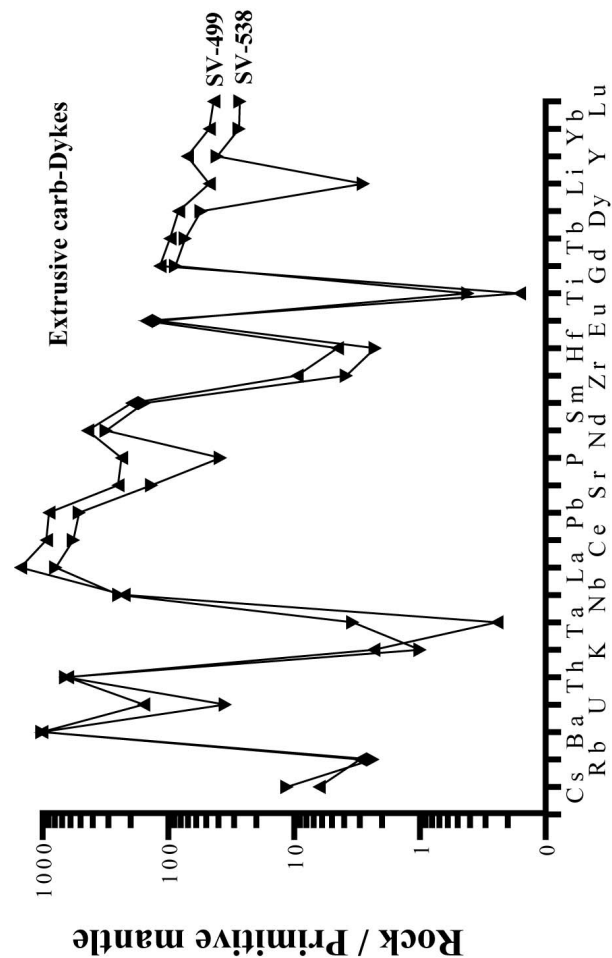

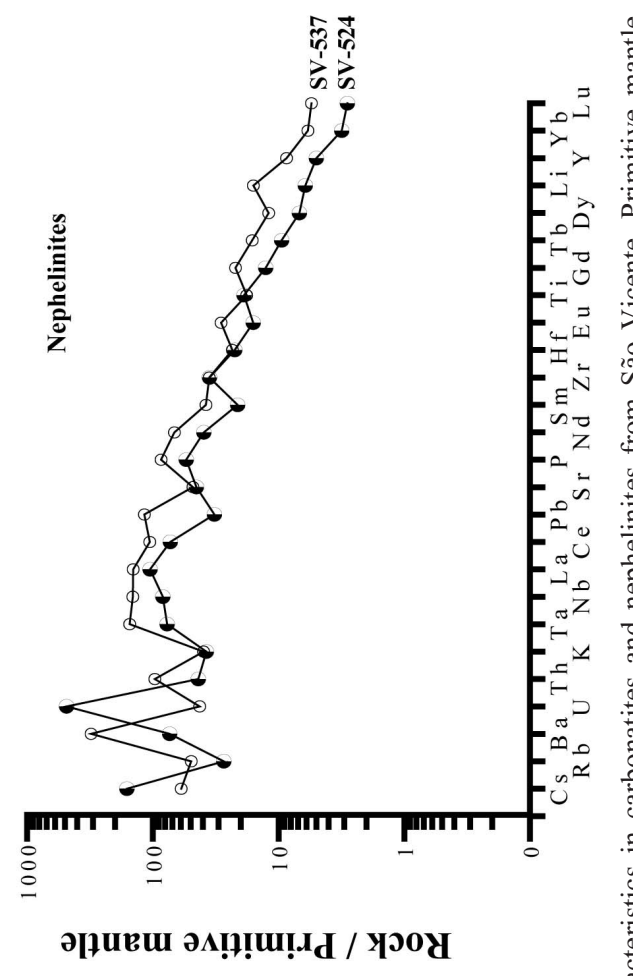

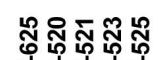

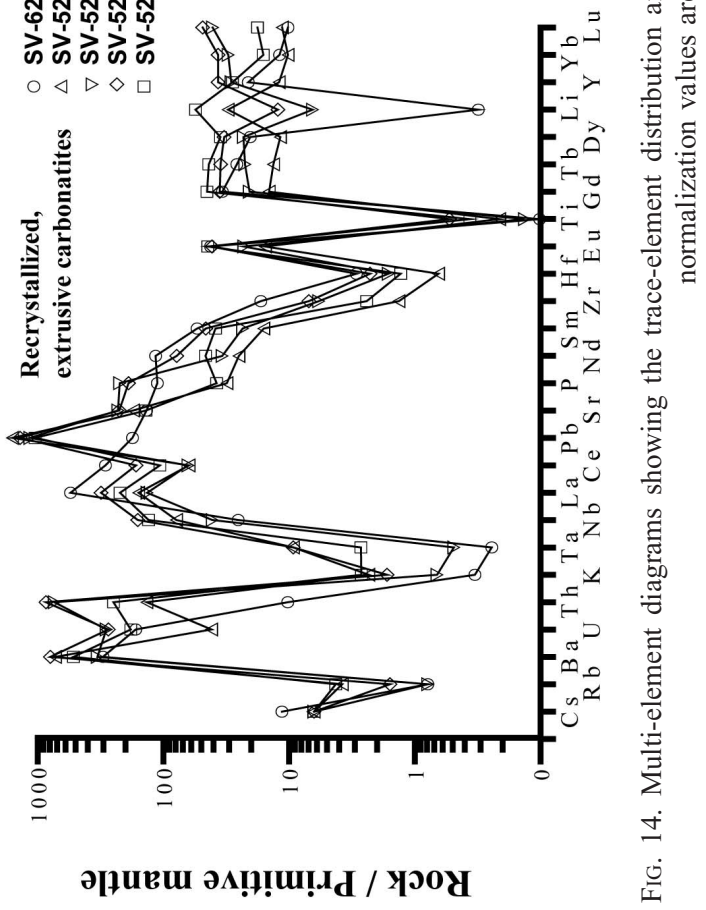


ones $(\mathrm{Cr}=20-9 \mathrm{ppm} ; \mathrm{Ni}=99-26 \mathrm{ppm} ; \mathrm{Co}=$ 14-4 ppm). In the intrusive carbonatites, the chromium and nickel contents are similar to those of the nephelinites, which range from $71-15 \mathrm{ppm}$ $\mathrm{Cr}$ and 110-43 ppm Ni (Table 18). Scandium concentrations are very low in all of the rock types, including the nephelinite, and with the low magnesium contents, this indicates that neither of the carbonatite groups or the nephelinites represent primary melts from a peridotite or wehrlite mantle.

The extrusive carbonatites, especially the less recrystallized ones, are severely depleted in $\mathrm{Rb}$, but $\mathrm{Rb}$ is less depleted in the intrusive rocks (Fig. 14), where it substitutes for $\mathrm{K}$ in biotite and phlogopite. The $\mathrm{Rb}$ contents in the intrusive carbonatites approach those in the nephelinites (Table 18).

The lithium content of most of the rocks is low and variable, it is concentrated in some of the extrusive carbonatites (sample SV-499, $\mathrm{Li}=$ 75 ppm; sample SV-525, Li = 89 ppm) which contain taeniolite, thus confirming the suggestion made in the mineral chemistry section that taeniolite is lithium rich. Barium is preferentially concentrated in the extrusive carbonatites, which contain up to 7000 ppm, mostly as baryte. Barium and thorium, have the largest positive anomalies in the extrusive carbonatites, excluding REE (Fig. 14). In contrast, the barium concentrations in the intrusive carbonatites are ten times lower and thorium and uranium are very similar to each other; the characteristic M-shaped pattern of the extrusive carbonatites is not reproduced in the intrusive types (Fig. 14). Strontium is moderately abundant in all the São Vicente carbonatites (2870-5300 ppm), but these values are less than the mean values for magnesiocarbonatite and calciocarbonatite (means of 7030 and 5640 ppm, respectively, Woolley and Kempe, 1989). This is probably due to the fact that carbonates in these rocks are depleted in this element with respect to apatite, in which it is concentrated.

Of the high field strength elements (HFSEs), niobium has the highest concentrations, particularly in the extrusive rocks (sample SV-499, $\mathrm{Nb}=$ 161 ppm; sample SV-538, Nb = 177 ppm), which contain pyrochlore-group minerals. In this case the pyrochlore-group mineral is pyrochlore sensu stricto (see mineral chemistry) with a low tantalum content. The pyrochlore-bearing carbonatites have a strong positive niobium anomaly and are relatively depleted in tantalum in multielement diagrams (Fig. 14).
In summary, the whole-rock geochemistry data lead us to conclude that the extrusive carbonatites and dykes group, and the recrystallized extrusive carbonatite group, have more similarities than differences and that the most pronounced difference is the higher REE content of the extrusive non-recrystallized group (Fig. 15). The observed decrease in REEs in the recrystallized carbonatites can be explained by the greater relative mobility of the heavy REEs in alteration processes (Walter et al., 1995). The lowest REE concentrations are found in the intrusive carbonatites, which seems to reinforce their affinity with the nephelinites in view of their respective REE patterns (Fig. 15). Based on the REE patterns, an origin of the São Vicente carbonatites by extreme fractionation from nephelinites can be inferred.

\section{Isotope geochemistry}

To constrain the possible origin of the São Vicente carbonatites and associated nephelinites, we analysed their radiogenic $(\mathrm{Sr}, \mathrm{Nd}, \mathrm{Pb})$ isotope compositions (Tables 19 and 20).

The strontium and neodymium isotope compositions of the carbonatites and nephelinites are similar and tightly grouped (Fig. 16). They plot to the left of the mantle array, close to the field of 'young' carbonatites and alkaline silicate rocks from East Africa. Our data are very similar to those obtained by Hoernle et al. (2002) in São Vicente, and are also somewhat similar to the intrusive calciocarbonatites of Fuerteventura, in the Canary Islands (de Ignacio et al., 2006). The São Vicente carbonatites and associated nephelinites have $\mathrm{Sr}$ and $\mathrm{Nd}$ isotope compositions that are close to the HIMU (high $\mu$; where $\mu$ is the ${ }^{238} \mathrm{U} /{ }^{204} \mathrm{~Pb}$ ratio) mantle component defined by Weaver (1991) and closer than those of Fuerteventura to the N-MORB component (Fig. 16).

Most of the lead isotope compositions of the São Vicente carbonatites and nephelinites lie beneath the northern hemisphere reference line (NHRL) of Hart (1984). Those samples that lie above the NHRL in the ${ }^{206} \mathrm{~Pb} /{ }^{204} \mathrm{~Pb} v s$. ${ }^{207} \mathrm{~Pb} /{ }^{204} \mathrm{~Pb}$ diagram are from the recrystallized extrusive carbonatites (Fig. 17). These samples also have anomalously low ${ }^{208} \mathrm{~Pb} /{ }^{204} \mathrm{~Pb}$ ratios. This might indicate that $\mathrm{Pb}$ was mobilized to a certain extent by fluids during recrystallization. The extrusive carbonatites and dykes that contain pyrochlore (samples SV-499 and SV-538) have 

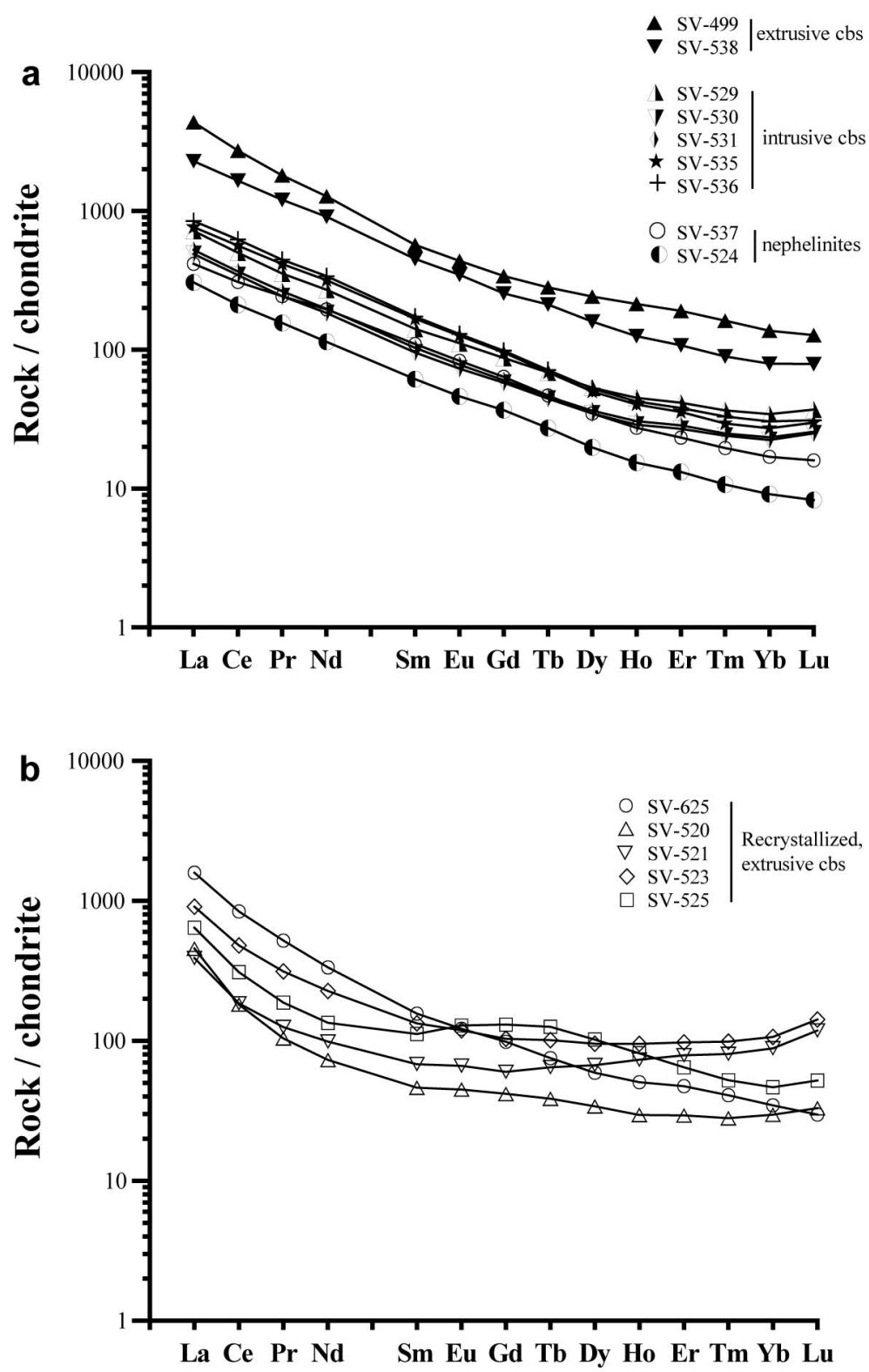

FIG. 15. Rare earth element data for São Vicente. (a) Rare earth element spectra for extrusive carbonatites, dykes, intrusive carbonatites and nephelinites. These data are normalized to the primitive mantle values of Sun and McDonough (1989). (b) The recrystallized extrusive carbonatites plotted in isolation for clarity.

lower lead concentrations (Table 18) than the extrusive recrystallized samples with neither modal pyrochlore nor any other lead-controlling mineral phase, which may be an indication that some lead has been added during recrystallization.
The lead isotope compositions of the other samples are tightly grouped, and are similar in both the carbonatites and nephelinites. As a whole, the São Vicente carbonatites and nephelinites plot closer to the HIMU component than 
TABLE 19. Rubidium-strontium and samarium-neodymium isotope ratios for the São Vicente carbonatites and nephelinites.

\begin{tabular}{lccccccc}
\hline Sample & Type & $\mathrm{Rb}(\mathrm{ppm})$ & $\mathrm{Sr}(\mathrm{ppm})$ & ${ }^{87} \mathrm{Rb} /{ }^{86} \mathrm{Sr}$ & ${ }^{87} \mathrm{Sr} /{ }^{86} \mathrm{Sr}$ & $\left({ }^{87} \mathrm{Sr} /{ }^{86} \mathrm{Sr}\right)_{\mathrm{i}}$ & ${ }^{\varepsilon S r}$ \\
\hline SV-499 & EC & 1.9 & 5,306 & 0.0010 & 0.703112 & 0.703112 & -20 \\
SV-538 & EC & 1.5 & 2,870 & 0.0015 & 0.703277 & 0.703277 & -17 \\
SV-521 & REC & 0.5 & 4,840 & 0.0003 & 0.703184 & 0.703184 & -19 \\
SV-523 & REC & 1.0 & 4,728 & 0.0006 & 0.703187 & 0.703187 & -19 \\
SV-529 & IC & 46.6 & 5,281 & 0.0255 & 0.703138 & 0.703137 & -19 \\
SV-531 & IC & 3.4 & 3,995 & 0.0025 & 0.703203 & 0.703203 & -18 \\
SV-537 & Neph & 31.4 & 1,014 & 0.0896 & 0.703390 & 0.703385 & -16 \\
& & & & & & & \\
\hline & & Sm (ppm) & Nd (ppm) & ${ }^{147} \mathrm{Sm} /{ }^{143} \mathrm{Nd}$ & ${ }^{143} \mathrm{Nd} /{ }^{144} \mathrm{Nd}$ & $\left({ }^{143} \mathrm{Nd} /{ }^{144} \mathrm{Nd}\right)_{\mathrm{i}}$ & \multirow{2}{*}{$\varepsilon \mathrm{Nd}$} \\
\hline SV-499 & EC & 86.9 & 594 & 0.0885 & 0.512891 & 0.512889 & +4.99 \\
SV-538 & EC & 68.9 & 423 & 0.0985 & 0.512891 & 0.512888 & +4.99 \\
SV-521 & REC & 10.4 & 46 & 0.1367 & 0.512888 & 0.512884 & +4.91 \\
SV-523 & REC & 20.4 & 106 & 0.1164 & 0.512896 & 0.512893 & +5.07 \\
SV-529 & IC & 21.6 & 126 & 0.1036 & 0.512892 & 0.512889 & +5.00 \\
SV-531 & IC & 15.8 & 91.7 & 0.1042 & 0.512892 & 0.512889 & +5.00 \\
SV-537 & Neph & 16.8 & 91.2 & 0.1114 & 0.512904 & 0.512901 & +5.23 \\
& & & & & & & \\
\hline
\end{tabular}

Abbreviations are: $\mathrm{EC}=$ extrusive carbonatite; $\mathrm{REC}=$ recrystallized, extrusive carbonatite; $\mathrm{IC}=$ intrusive carbonatite; Neph $=$ nephelinite.

Those values identified with an $\mathrm{i}$ are initial ratios calculated for $4 \mathrm{Ma}$.

the carbonatites from Fuerteventura (Fig. 17), reflecting enrichment in the ${ }^{206} \mathrm{~Pb} /{ }^{204} \mathrm{~Pb}$ isotope ratios. Such enrichment is best illustrated in plots of ${ }^{206} \mathrm{~Pb} /{ }^{204} \mathrm{~Pb}$ vs. ${ }^{87} \mathrm{Sr} /{ }^{86} \mathrm{Sr}$ and ${ }^{143} \mathrm{Nd} /{ }^{144} \mathrm{Nd}$ (Fig. 18a). In the lead $v s$. strontium diagram, the São Vicente carbonatites and nephelinites plot on a mixing line between HIMU and enriched mantle 1 (EM1) mantle components. In common with the Fuerteventura carbonatites, the São Vicente carbonatites have FOZO-like strontium isotope compositions that are displaced to the right of the FOZO (focus zone) field due to enrichment in ${ }^{206} \mathrm{~Pb} /{ }^{204} \mathrm{~Pb}$. This could indicate that there is no contribution from EM1, which is clearly seen in the ${ }^{206} \mathrm{~Pb} /{ }^{204} \mathrm{~Pb}$ vs. ${ }^{143} \mathrm{Nd} /{ }^{144} \mathrm{Nd}$ plot (Fig. 18b), where the São Vicente samples fall on the same mixing trend between FOZO and HIMU as the Fuerteventura carbonatites and alkaline silicate rocks.

Gerlach et al. (1988), in a study of isotopic compositions of the Cape Verde archipelago, observed that the northern islands were characterized by isotopic signatures that are mixtures between a HIMU component dominating lead isotope compositions and a DM (depleted mantle) component. This depleted mantle component can be identified as a deep mantle, FOZO-type source, taking into account the resemblance between the São Vicente and Fuerteventura isotope compositions in carbonatites and their accompanying alkaline silicate rocks. This hypothesis is supported by helium isotope data from the northern Cape Verde Islands (Christensen et al., 2001; Doucelance et al., 2003; Mata et al., 2010). The ${ }^{4} \mathrm{He} /{ }^{3} \mathrm{He}$ ratios reported are relatively low, and indicate a non-degassed source in the lower mantle.

As in Fuerteventura, the origin of the HIMU mantle component in the São Vicente carbonatites and nephelinites is difficult to explain. There are three possibilities: (1) recycling of subducted oceanic crust into the mantle (Hofmann and White, 1982; Weaver, 1991; Chauvel et al., 1992; Hofmann, 1997); (2) contributions from a subcontinental lithospheric mantle (SCLM), as proposed by Hawkesworth et al. (1990), or (3) uranium enrichment over lead in mantle clinopyroxene produced by metasomatizing low degree melts (Pilet et al., 2005). Alternatively, Doucelance et al. (2010) proposed that the Cape Verde ocean lithosphere acted as the depleted component, and that the enriched component was 
a result of metasomatism derived from subduction of oceanic crust and sediments at around $400 \mathrm{Ma}$ during closure of the Rheic Ocean.

\section{Discussion}

The rock textures, mineralogy and geochemistry show that there are two distinct groups of carbonatite in São Vicente, extrusive Mg-rich carbonatites and intrusive calciocarbonatites. They are spatially associated with nephelinites and an evaluation of relationship between the nephelinites and carbonatites is therefore necessary.

There are three probable mechanisms for the formation of carbonatites: (1) direct melting from carbonate-rich mantle peridotite or wehrlite (Wallace and Green, 1988; Eggler, 1989, Wyllie and Lee, 1998; Dalton and Wood, 1993); (2) extensive fractional crystallization from alkaline silicate magmas (Wyllie and Lee, 1998; Veksler et al., 1998); (3) liquid immiscibility from $\mathrm{CO}_{2}$-rich, alkaline silicate magmas (Koster van Groos and Wyllie, 1963; Kjarsgaard and Hamilton, 1989; Kjarsgaard et al., 1995; Lee and Wyllie, 1996).

The first of these mechanisms, as a direct melt from the mantle, can be discarded in our opinion for São Vicente. Although the non-recrystallized extrusive rocks are magnesiocarbonatites (Fig. 13), with $<1$ wt.\% $\mathrm{SiO}_{2}$ and high $(\mathrm{Mg} /(\mathrm{Mg}+\mathrm{Fe}))$ ratios (Table 18$)$, they contain low concentrations of compatible elements (such as $\mathrm{Cr}$ and $\mathrm{Ni}$ ), which should be relatively enriched in melts in equilibrium with carbonate-rich peridotite. For comparison, the mantle-derived magnesiocarbonatitic lapilli from Tamazert in Morocco have consistently higher $\mathrm{MgO}$ contents (16-18 wt.\%) and 650-2120 ppm Cr (Mourtada et al., 1997), whereas the São Vicente extrusive carbonatites and dykes contain $\leqslant 10$ wt. $\% \mathrm{MgO}$; 4-15 ppm Cr, and 10-18 ppm Ni (Table 18). Their $\mathrm{Ca} / \mathrm{Mg}$ ratio is also rather high with values of 3.8 and 6.3 in samples SV-499 and SV-538, respectively, compared to $1.5-1.8$ in the magnesiocarbonatitic lapilli from Morocco (Mourtada et al., 1997). The compositions of experimental carbonate melts in equilibrium with suboceanic peridotites were compared by Kogarko (1993) with two carbonatite samples, one from São Vicente and one from São Tiago. Based on the close match between them, the author concluded that, "at least some of the dolomitic carbonatites of the Cape Verde Islands" could be derived from 

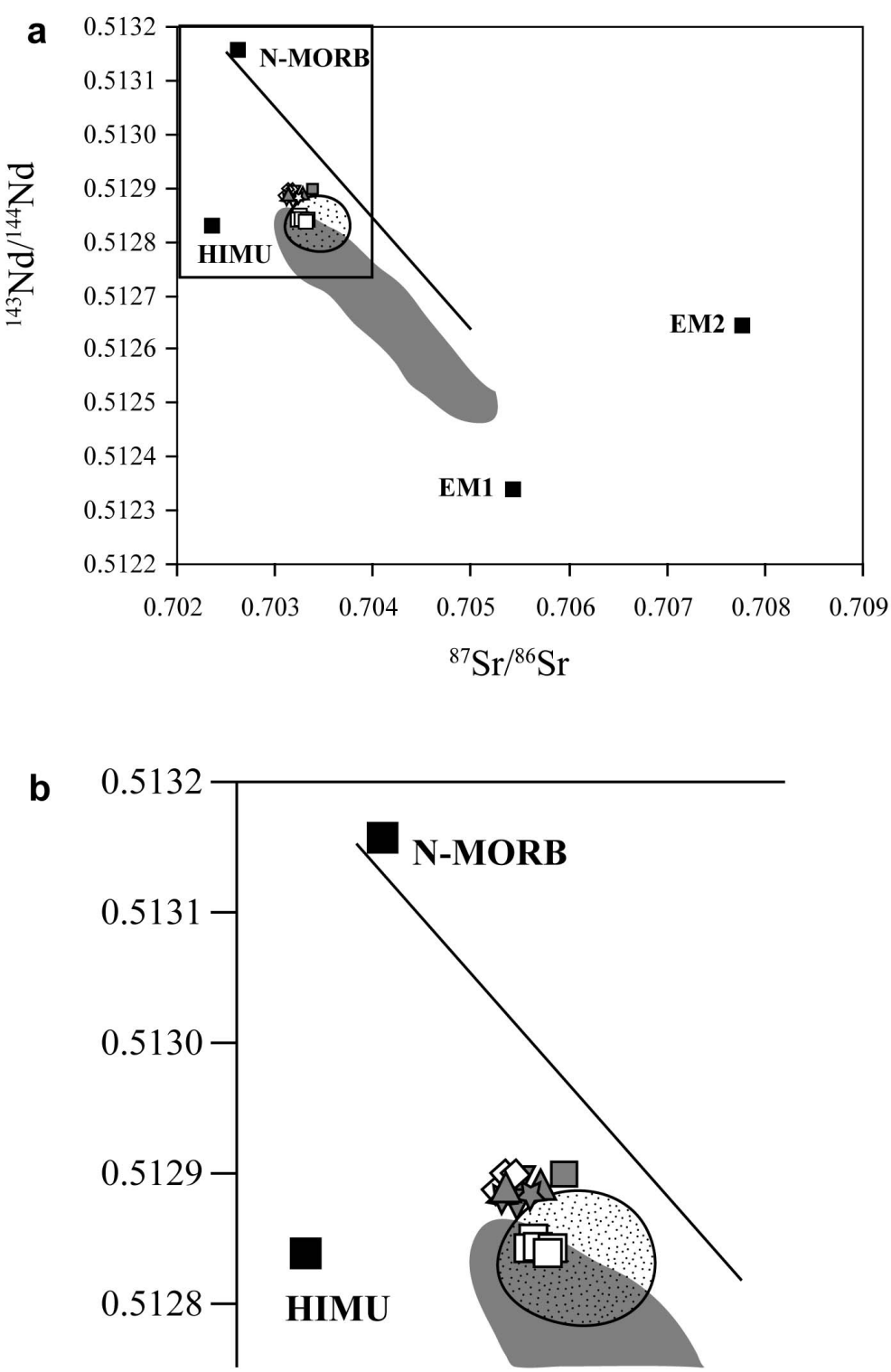

FIG. 16. Strontium-neodymium isotope data. (a) Strontium-neodymium initial isotope ratios for the São Vicente carbonatites and nephelinites. Mantle components are from Hart (1988) and the straight line corresponds to the mantle array (dePaolo and Wasserburg, 1976). The grey shaded area represents the compositions of carbonatites and alkaline-silicate rocks from the East African Rift, with data taken from Bell and Blenkinsop (1987); Bell and Dawson (1995); Bell and Simonetti (1996); Kalt et al. (1997) and Bell and Tilton (2001). The grey symbols represent rocks from São Vicente: upward-facing triangles = extrusive carbonatites and dykes; downward-facing triangles $=$ recrystallized extrusive carbonatites; stars $=$ intrusive carbonatites; squares $=$ nephelinites. The white symbols are taken from other studies: diamonds = data from Hoernle et al. (2002) for São Vicente carbonatites; squares and stippled area $=$ Fuerteventura carbonatites and alkaline silicate rocks, respectively (de Ignacio et al., 2006). (b) An enlarged portion of the diagram showing how tightly grouped the isotopic compositions of both the carbonatites and nephelinites are. 

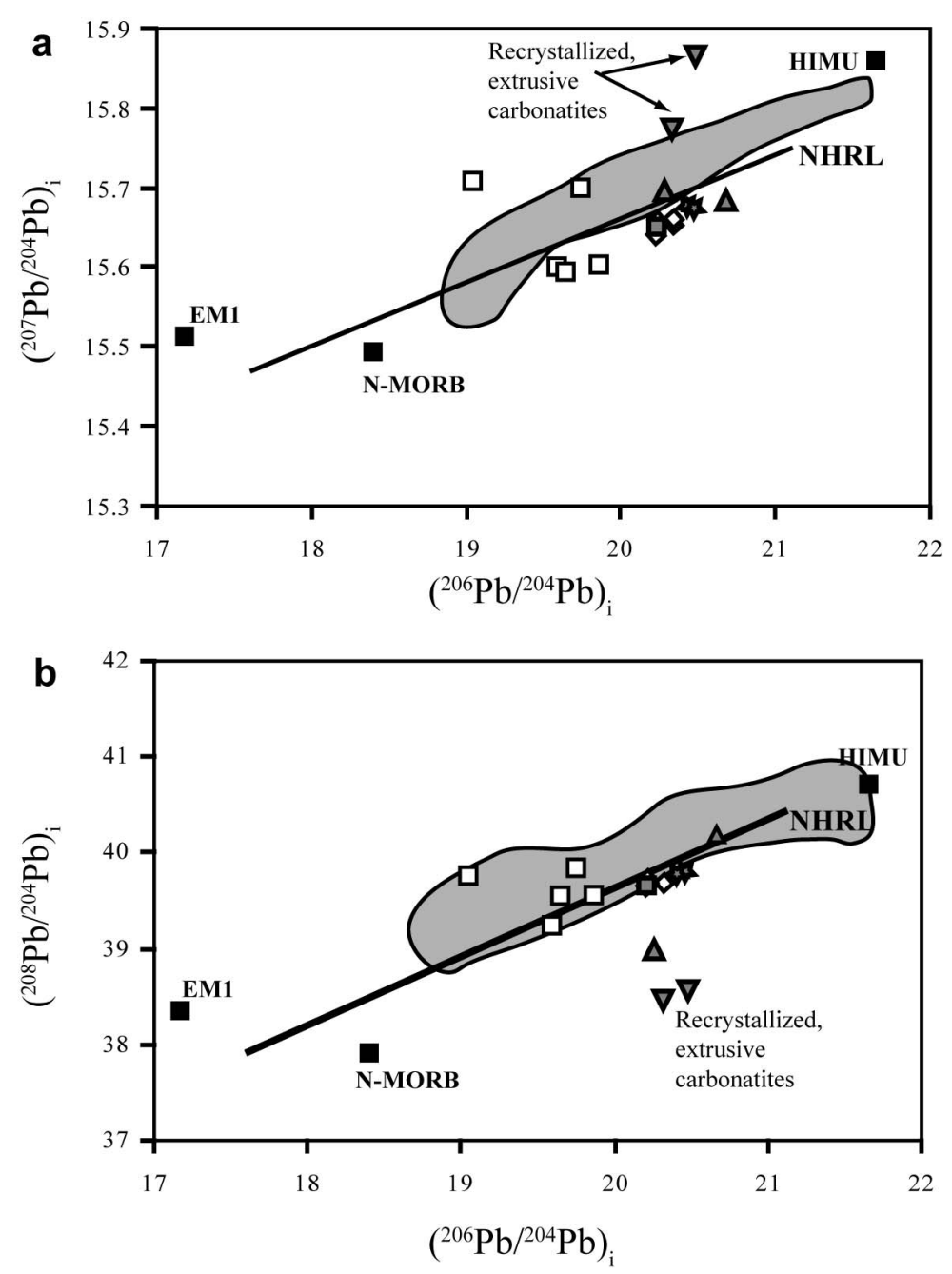

FIG. 17. Lead isotope compositions of the São Vicente carbonatites and nephelinites. The grey symbols represent rocks from São Vicente: upward-facing triangles = extrusive carbonatites and dykes; downward-facing triangles = recrystallized extrusive carbonatites; stars $=$ intrusive carbonatites; squares $=$ nephelinites. The northern hemisphere reference line (NHRL) is from Hart (1984).

direct partial melting of the mantle. However, these compositions are substantially richer in $\mathrm{MgO}(14-16$ wt.\%) and poorer in $\mathrm{CaO}(21-29$ wt.\%) than samples SV-499 and SV-538, which contain $\leqslant 10$ wt. $\% \mathrm{MgO}$ and $39-44$ wt. $\% \mathrm{CaO}$.

The possibility that the São Vicente carbonatites were derived from extensive fractional crystallization of a nephelinite parent magma is supported by the field relationships and by the mineralogical and geochemical affinities between the intrusive carbonatites and nephelinites. Early crystallization of Ti-rich clinopyroxene, rhönite and Fe-Ti oxides in the nephelinites would strongly deplete the remaining melt in $\mathrm{TiO}_{2}$, which would explain the sharp negative titanium anomalies in the carbonatite multi-element diagrams (Fig. 14). Later fractionation of nepheline and kaersutite might explain the alkali depletion in the carbonatites (the strong negative $\mathrm{K}$ anomalies in Fig. 14). However, fractional crystallization alone cannot explain all of geochemical differences between the intrusive 

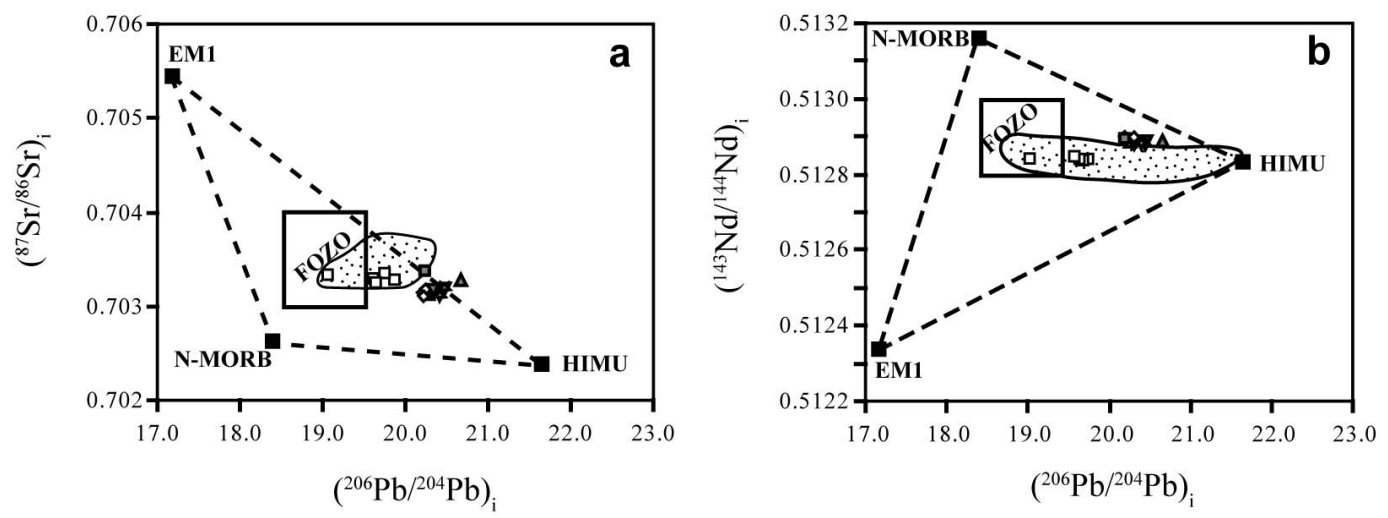

FIG. 18. The ${ }^{206} \mathrm{~Pb} /{ }^{204} \mathrm{~Pb}$ initial ratio plotted $v s .(a){ }^{87} \mathrm{Sr} /{ }^{86} \mathrm{Sr}$ and $(b){ }^{143} \mathrm{Nd} /{ }^{144} \mathrm{Nd}$. The box represents the FOZO (Focus Zone) mantle component, defined by Hart et al. (1992) and Hauri et al. (1994). The grey symbols represent rocks from São Vicente: upward-facing triangles = extrusive carbonatites and dykes; downward-facing triangles = recrystallized extrusive carbonatites; stars $=$ intrusive carbonatites; squares $=$ nephelinites. The white symbols are taken from other studies: diamonds = data from Hoernle et al. (2002) for São Vicente carbonatites; squares and stippled area $=$ Fuerteventura carbonatites and alkaline silicate rocks, respectively (de Ignacio et al., 2006).

and extrusive carbonatites, for example, the very high $\mathrm{Ba}$ contents in extrusive carbonatites or the difference between the $\mathrm{Mg}$ and $\mathrm{Nb}$ contents in the intrusive and extrusive carbonatites.

An evaluation of the immiscibility mechanism can be obtained by plotting both the carbonatitic (extrusive and intrusive) and nephelinitic compositions in experimental diagrams. In Fig. 19, the nephelinite compositions plot inside the twoliquid field, whereas the extrusive carbonatites and dykes plot inside the field of a single carbonatitic liquid. In immiscible melts, $\mathrm{Ba}, \mathrm{Sr}$ and $R E E$ partition into the carbonatite fraction; $\mathrm{Zr}$ tends to remain with the silicate fraction but $\mathrm{Nb}$ moves into the carbonatite (Veksler et al., 1998). This is observed in multi-element diagrams which compare the nephelinites with extrusive carbonatites and dykes and with intrusive carbonatites (Fig. 20). The intrusive carbonatites plot to the left of the "one liquid, carbonatitic" field in Fig. 19. We interpret these rocks as cumulates that crystallized from an immiscible carbonatite fraction in the early stages of differentiation, when calcite was the liquidus carbonate phase due to the high calcium activity in the system. In a study of the $\mathrm{CaO}-\mathrm{MgO}-\mathrm{SiO}_{2}-\mathrm{CO}_{2}-\mathrm{H}_{2} \mathrm{O}$ system, Otto and Wyllie (1993) showed that calcite forms as the liquidus phase followed, at lower temperatures, by co-crystallization of calcite and dolomite. In São Vicente, the extrusive carbonatites and dykes represent products of the carbonatite fraction that crystallized slightly later than cumulates, in a stage dominated by a high enrichment in a vapour (volatile) phase. Thus, dolomite would join calcite as a precipitating phase, and the high volatile content would be reflected in: (1) the modal abundance of apatite (high $\mathrm{P}_{2} \mathrm{O}_{5}$ ); (2) the high fluorine contents in this apatite; (3) relatively high $\mathrm{SO}_{3}$ contents forming abundant baryte. In this kind of crystallization process, nucleation would be fast, giving raise to the microgranular aggregates of each of the three phases (apatite, calcite and dolomite), that form the bands in the extrusive carbonatites and dykes. Apatite would start to crystallize in the early carbonatite fractionation stage, together with calcite, and would continue to form in the vapour-dominated stage. However, it would sharply change its composition, and be strongly enriched in volatiles (fluorine) in the extrusive carbonatites and dykes. The REE, which are mostly controlled by apatite in both nephelinites and carbonatites, increase progressively from nephelinites (accessory apatite) to intrusive carbonatites (essential apatite) and extrusive carbonatites (Fig. 20). Lastly, niobium would remain as an incompatible element during apatite and carbonate crystallization, whereas later it would be stabilized in pyrochlore in the extrusive carbonatites and dykes.

A cartoon depicting our model is shown in Fig. 21. It explains the complex variations observed in the mineralogy and geochemistry of the rocks, and is consistent with field relations, 


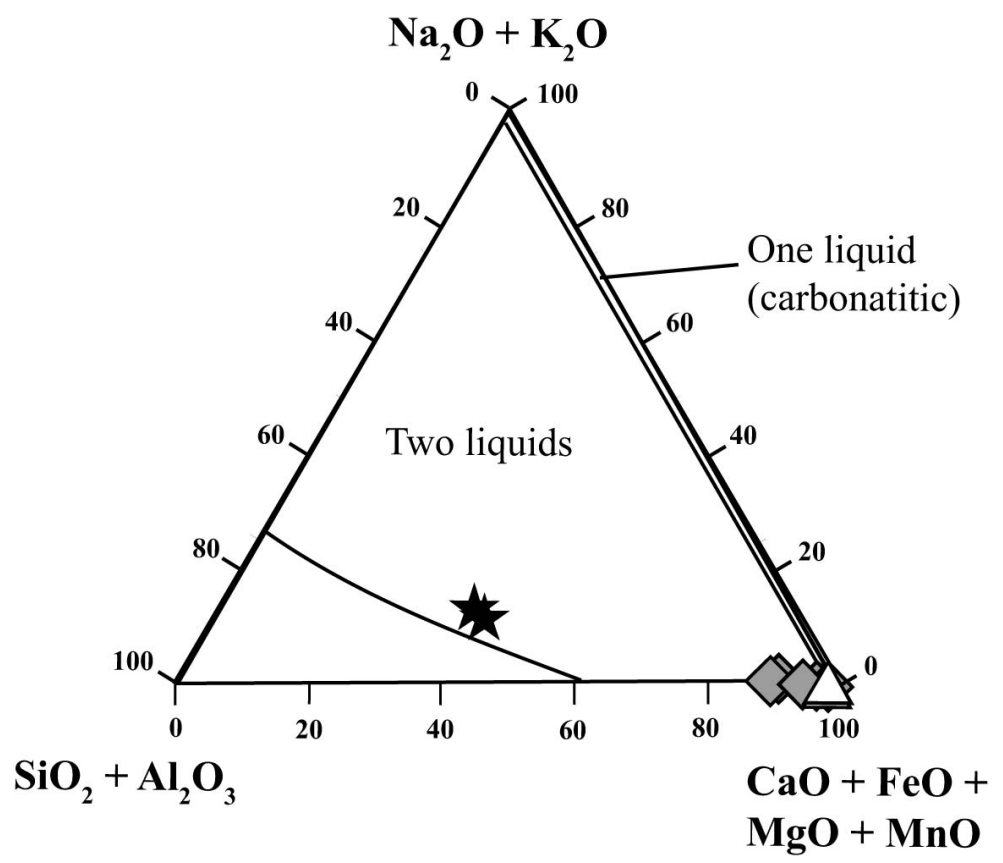

FIG. 19. Immiscibility diagram from Kjarsgaard and Hamilton (1989) in which the São Vicente nephelinites plot in the 'two-liquid' field, but extrusive carbonatites plot in the 'one liquid, carbonatitic' field. The silicate limb of immiscibility (solid line) corresponds to $0.5 \mathrm{GPa}$. The symbols are: black stars $=$ nephelinites; grey diamonds $=$ intrusive carbonatites; white triangles = extrusive carbonatites and dykes

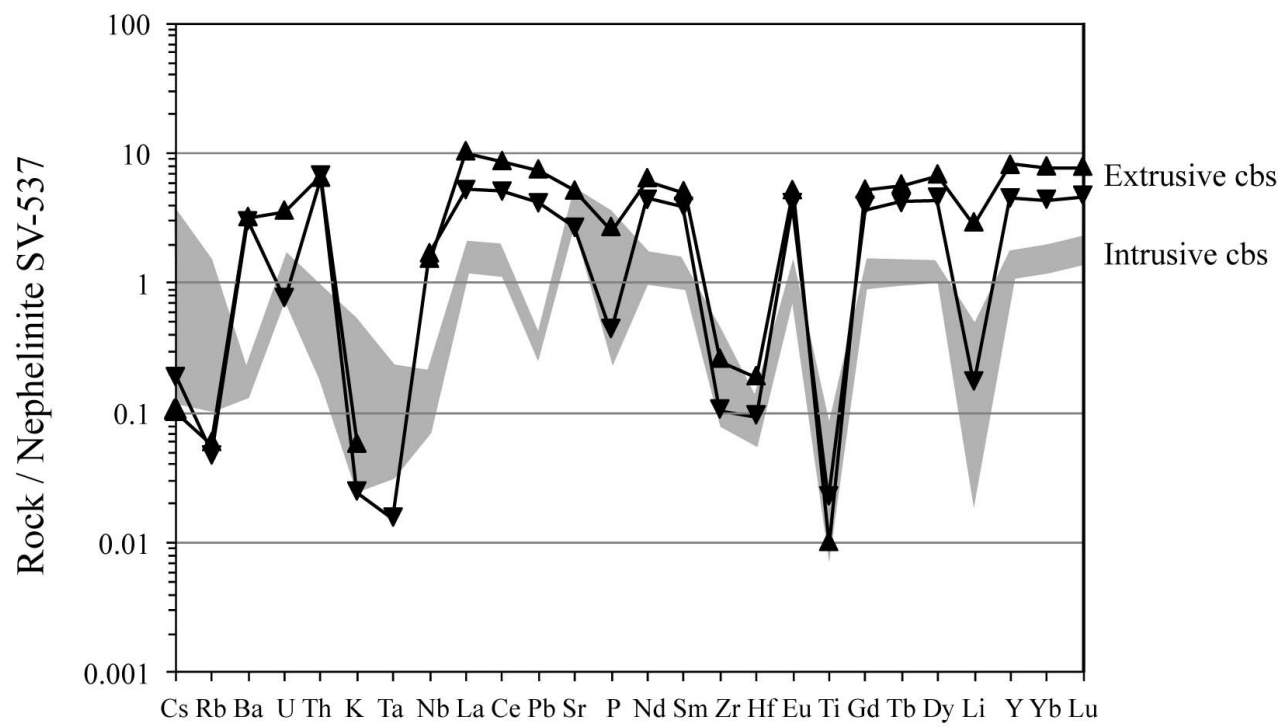

FIG. 20. Comparison of the multi-element patterns of the carbonatites and nephelinites from São Vicente. Primitive mantle normalization values are from Sun and McDonough (1989). The abbreviation cbs $=$ carbonatites. 


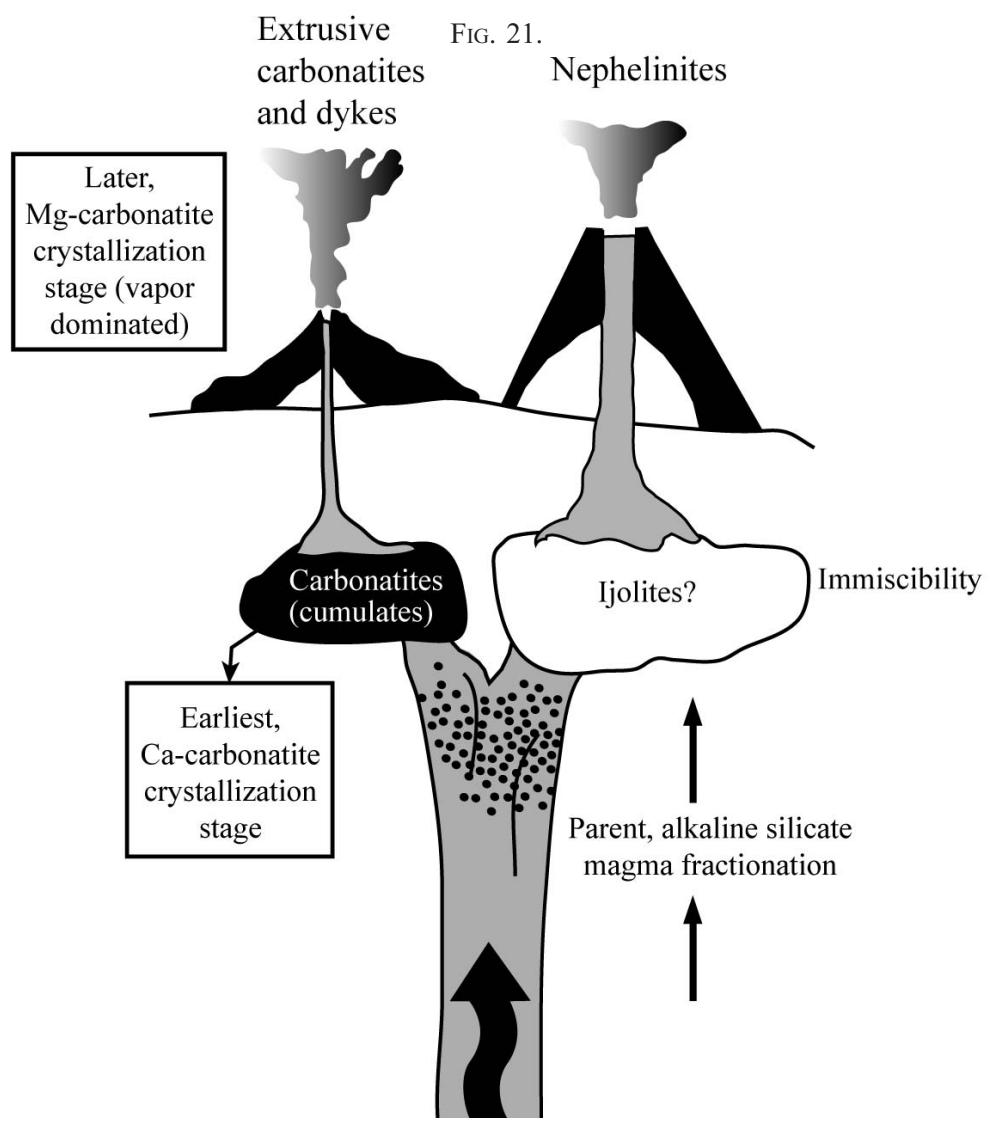

Fig. 21. Cartoon (not drawn to scale) illustrating the proposed genetic model for the São Vicente carbonatites and nephelinites.

explaining both the close association of intrusive carbonatites (apophyses) and extrusive carbonatites (lava flows) plus dykes and the close association of nephelinites and carbonatites. There may have been zones where both magmas interacted, which would explain some of the reactions observed in minerals from the intrusive carbonatites, the compositions of which are similar to those in the nephelinites.

\section{Conclusions}

Two groups of carbonatites can be distinguished in São Vicente on the basis of the field relationships, rock textures, mineral chemistry and whole-rock geochemistry: intrusive sövites, and extrusive magnesiocarbonatites and related dykes. Some of the latter show evidence that recrystallization has affected the textures, chemical composition of the earliest phases (carbonates and apatite) and probably lead isotope compositions. The extrusive carbonatites contain some unusual minerals, including $\mathrm{Na}$ - and Sr-rich fluorapatite and Li-rich mica that, to our knowledge, are reported here for the first time.

The nephelinites that are associated with carbonatites are of two mineralogical types: rhönite-bearing nephelinites and amphibolemica-bearing nephelinites. Conditions for rhönite stability coupled with geothermobarometry on ilmenite-magnetite pairs indicate that the rhönite-bearing nephelinites formed at a higher temperature and less volatile saturation conditions than amphibole-mica-bearing nephelinites.

The whole-rock geochemistry reveals an affinity between the intrusive carbonatites and the nephelinites. Multi-element diagrams and $R E E$ patterns are consistent with the progressive 
fractionation of silicates as a control on the whole-rock geochemistry. The potassium content is influenced by the fractionation of amphiboles and mica in the nephelinites and by the fractionation of mica in the sövites. Titanium is strongly depleted in the carbonatites due to the partitioning of $\mathrm{Ti}$ into $\mathrm{Ti}$-rich clinopyroxene, rhönite, ilmenite-magnetite and, to a lesser extent, mica.

The extrusive carbonatites strongly concentrate: (1) large-ion lithiophile elements, especially barium and lithium, which is reflected in the presence of lithium-rich taeniolite and baryte; (2) volatiles, as shown by their high $\mathrm{P}, \mathrm{F}$ and $\mathrm{S}$ content; (3) REE, Th and $\mathrm{Nb}$, which are present in pyrochlore.

The proposed model for the genesis of the carbonatites and nephelinites involves an alkaline-silicate parent magma rich in $\mathrm{CO}_{2}$ from which nephelinites crystallized at low pressure and high temperature. Fractionation concentrated volatiles in the magma and an immiscible carbonatite separated. Calcite-dominated cumulates containing a few silicates crystallized at an early stage. Subsequently, in the residual carbonatite fraction, calcite and dolomite and apatite crystallized rapidly in vapour (volatile) dominated conditions, the rapid nucleation rates produced a characteristic banded texture. Radiogenic isotope compositions for both carbonatites and nephelinites are very tightly grouped and are consistent with this model.

\section{Acknowledgements}

This work was funded by the research projects CGL2009-07946 of the Spanish Government and GR58/08 of the Universidad Complutense de Madrid. We are grateful for the invaluable help of A. Fernández-Larios and J. González del Tánago with microprobe analyses and imaging, and to C. Galindo and the Centro de Geocronología y Geoquímica Isotópica, for analyses of radiogenic isotope compositions. The manuscript was improved by the comments and suggestions of L.N. Kogarko, an anonymous referee and Hilary Downes.

\section{References}

Alves, M.C.A., Macedo, J.R., Silva, L.C., Serralheiro, A. and Peixoto Faria, A.F. (1979) Estudo geológico, petrológico e vulcanológico da ilha de Santiago (Cabo Verde). Garcia de Orta, 3, 47-74.
Ancochea, E., Huertas, M.J., Hernán, F. and Brändle, J.L. (2010) Volcanic evolution of São Vicente, Cape Verde Islands: the Praia Grande landslide. Journal of Volcanology and Geothermal Research, 198, $143-157$.

Andersen, D.J. and Lindsley, D.H. (1985) New (and final) models for the Ti-magnetite/ilmenite geothermometer and oxygen barometer. EOS Transactions, American Geophysical Union, 66, 416.

Assunção, C.F.T., Machado, F. and Gomes, R.A.D. (1965) On the occurrence of carbonatites in the Cape Verde Islands. Boletim da Sociedade Geológica de Portugal, 16, 179-188.

Atencio, D., Andrade, M.B., Christy, A.G., Gieré, R. and Kartashov, P.M. (2010) The pyrochlore supergroup of minerals: nomenclature. The Canadian Mineralogist, 48, 673-698.

Bell, K. and Blenkinsop, J. (1987) $\mathrm{Nd}$ and $\mathrm{Sr}$ isotopic compositions of East African carbonatites: implications for mantle heterogeneity. Geology, 15, 99-102.

Bell, K. and Dawson, J.B. (1995) Peralkaline nephelinite/carbonatite liquid immiscibility: comparison of phase composition in experiments and natural lavas from Oldoinyo Lengai. Pp. 100-113 in: Carbonatite Volcanism: Oldoinyo Lengai and the Petrogenesis of Natrocarbonatites. (K. Bell and J. Keller, editors). IAVCEI Proceedings in Volcanology, 4. Springer, Berlin.

Bell, K. and Simonetti, A. (1996) Carbonatite magmatism and plume activity: implications for the $\mathrm{Nd}, \mathrm{Pb}$ and $\mathrm{Sr}$ isotope systematics of Oldoinyo Lengai. Journal of Petrology, 37, 1321-1339.

Bell, K. and Tilton, G.R. (2001) Nd, Pb and $\mathrm{Sr}$ isotopic compositions of East African carbonatites: evidence for mantle mixing and plume inhomogeneity. Journal of Petrology, 42, 1927-1945.

Brassinnes, S., Balaganskaya, E. and Demaiffe, D. (2005) Magmatic evolution of the differentiated ultramafic, alkaline and carbonatite intrusion of Vuoriyarvi (Kola Peninsula, Russia): a LA-ICP-MS study of apatite. Lithos, 85, 76-92.

Chakhmouradian, A.R. and Williams, C.T. (2004) Mineralogy of high-field-strength elements ( $\mathrm{Ti}, \mathrm{Nb}$, $\mathrm{Zr}$, Ta, Hf) in phoscoritic and carbonatitic rocks of the Kola Peninsula, Russia. Pp. 294-340 in: Phoscorites and Carbonatites, from mantle to mine: The key example of the Kola Alkaline Province, (F. Wall and A.N. Zaitsev, editors). The Mineralogical Society, London.

Chauvel, C., Hofmann, A.W. and Vidal, P. (1992) HIMU-EM: the French Polynesian connection. Earth and Planetary Science Letters, 110, 99-119.

Christensen, B.P., Holm, P.M., Jambon, A. and Wilson, J.R. (2001) Helium, argon and lead isotopic composition of volcanics from Santo Antao and 
Fogo, Cape Verde Islands. Chemical Geology, 178, $127-142$.

Cooper, A.F. and Patterson, L.A. (1995) Lithium in carbonatites - consequence of an enriched mantle source? Mineralogical Magazine, 59, 401-408.

Dalton, J.A. and Wood, B.J. (1993) The compositions of primary carbonate melts and their evolution through wallrock reaction with the mantle. Earth and Planetary Science Letters, 119, 511-525.

De Ignacio, C., Muñoz, M., Sagredo, J., FernándezSantín, S. and Johansson, A. (2006) Isotope geochemistry and FOZO mantle component of the alkaline-carbonatitic association of Fuerteventura, Canary Islands, Spain. Chemical Geology, 232, 99-113.

De Paolo, D.J. and Wasserburg, G.J. (1976) Nd isotopic variations and petrogenetic models. Geophysical Research Letters, 3, 249-252.

Doucelance, R., Escrig, S., Moreira, M., Gariépy, C. and Kurz, M.D. (2003) $\mathrm{Pb}-\mathrm{Sr}-\mathrm{He}$ isotope and trace element geochemistry of the Cape Verde Archipelago. Geochimica et Cosmochimica Acta, 67, 3717-3733.

Doucelance, R., Hammouda, T., Moreira, M. and Martins, J.C. (2010) Geochemical constraints on depth of origin of oceanic carbonatites: the Cape Verde case. Geochimica et Cosmochimica Acta, 74, $7261-7282$.

Eggler, D.H. (1989) Carbonatites, primary melts, and mantle dynamics. Pp. 561-579 in: Carbonatites: Genesis and Evolution, (K. Bell, editor). Unwin Hyman, London.

Gerlach, D.C., Cliff, R.A., Davies, G.R., Norry, M. and Hodgson, N. (1988) Magma sources of the Cape Verdes archipelago: isotopic and trace element constraints. Geochimica et Cosmochimica Acta, 52, 2979-2992.

Hart, S.R. (1984) A large-scale isotope anomaly in the Southern Hemisphere mantle. Nature, 309, $753-757$.

Hart, S.R. (1988) Heterogeneous mantle domains: signatures, genesis and mixing chronologies. Earth and Planetary Science Letters, 90, 273-296.

Hart, S.R., Hauri, E.H., Oschmann, L.A. and Whitehead, J.A. (1992) Mantle plumes and entrainment: isotopic evidence. Science, 256, 517-520.

Hauri, E.H., Whitehead, J.A. and Hart, S.R. (1994) Fluid dynamic and geochemical aspects of entrainment in mantle plumes. Journal of Geophysical Research, 99, 24275-24300.

Hawkesworth, C.J., Kempton, P.D., Rogers, N.W., Ellam, R.M. and van Calsteren, P.W. (1990) Continental mantle lithosphere and shallow level enrichment processes in the Earth's mantle. Earth and Planetary Science Letters, 96, 256-268.

Henderson, C.M.B. and Gibb, F.G.F. (1983) Felsic mineral crystallization trends in differentiating alkaline basic magmas. Contributions to Mineralogy and Petrology, 84, 355-364.

Hodgson, N.A. and Le Bas, M.J. (1992) The geochemistry and cryptic zonation of pyrochlore from San Vicente, Cape Verde Islands. Mineralogical Magazine, 56, 201-214.

Hoernle, K., Tilton, G., Le Bas, M.J., Duggen, S. and Garbe-Schönberg, D. (2002) Geochemistry of oceanic carbonatites compared with continental carbonatites: mantle recycling of oceanic crustal carbonate. Contributions to Mineralogy and Petrology, 142, 520-542.

Hogarth, D.D. (1977) Classification and nomenclature of the pyrochlore group. American Mineralogist, 62, 403-410.

Holm, P.M., Grandvuinet, T., Friis, J., Wilson, J.R., Barker, A.K. and Plesner, S. (2008) $\mathrm{An}{ }^{40} \mathrm{Ar}-{ }^{39} \mathrm{Ar}$ study of the Cape Verde hot spot: temporal evolution in a semistationary plate environment. Journal of Geophysical Research, 113, B08201, http:// dx.doi.org/10.1029/2007JB005339.

Hofmann, A.W. (1997) Mantle geochemistry: the message from oceanic volcanism. Nature, 385, 219-229.

Hofmann, A.W. and White, W.M. (1982) Mantle plumes from ancient oceanic crust. Earth and Planetary Science Letters, 57, 421-436.

Huertas, M.J., Hernán, F., Ancochea, E. and Brändle, J.L. (2006) El Edificio Antiguo de la isla de San Vicente (Cabo Verde): características del sector occidental. Geogaceta, 40, 95-98.

Jarosevitch, E.J. and Boatner, L.A. (1991) Rare-earth element reference samples for electron microprobe analysis. Geostandards Newsletter, 15, 397-399.

Jarosevitch, E.J., Nelen, J.A. and Norberg, J.A. (1980) Reference samples for electron microprobe analysis. Geostandards Newsletter, 4, 43-47 and 257-258.

Kalt, A., Hegner, E. and Satir, M. (1997) Nd, Sr, and Pb isotopic evidence for diverse lithospheric mantle sources of East African Rift carbonatites. Tectonophysics, 278, 31-45.

Kjarsgaard, B.A. and Hamilton, D.L. (1989) The genesis of carbonatites by immiscibility. Pp. 388-404 in: Carbonatites: Genesis and Evolution, (K. Bell, editor). Unwin Hyman, London.

Kjarsgaard, B.A., Hamilton, D.L. and Peterson, T.D. (1995) Peralkaline nephelinite/carbonatite liquid immiscibility: comparison between phase compositions in experiments and natural lavas. Pp. 163-190 in: Carbonatite Volcanism: Oldoinyo Lengai and the petrogenesis of natrocarbonatites. (K. Bell and $\mathrm{J}$. Keller, editors). Springer-Verlag, Berlin.

Kogarko, L.N. (1993) Geochemical characteristics of oceanic carbonatites from the Cape Verde Islands. South African Journal of Geology, 96, 119-125. 
Kogarko, L.N., Hellebrand, E. and Ryabchikov, I.D. (2005) Trace element partitioning between rhönite and silicate melt in Cape Verde volcanics. Geochemistry International, 43, 1-7.

Koster van Groos, A.F. and Wyllie, P.J. (1963) Experimental data bearing on the role of liquid immiscibility in the genesis of carbonatites. Nature, 199, 801-802.

Le Bas, M.J. and Handley, C.D. (1979) Variation in apatite composition in ijolitic and carbonatitic igneous rocks. Nature, 279, 54-56.

Lee, W.J. and Wyllie, P.J. (1996) Liquid immiscibility in the join $\mathrm{NaAlSi}_{3} \mathrm{O}_{8}-\mathrm{CaCO}_{3}$ and the origin of calciocarbonatite magmas. Journal of Petrology, 37, $1125-1152$.

Lepage, L.D. (2003) ILMAT: an excel worksheet for ilmenite-magnetite geothermometry and geobarometry. Computers and Geosciences, 29, 673-678.

Machado, F., Leme, J., Mojardino, J. and Seita, M.F. (1968) Carta geologica de Cabo Verde, noticia explicativa da folha da ilha Brava e dos ilheus Secos (na escala 1/5000). Garcia de Orta, 16, 123-130.

Mata, J., Moreira, M., Doucelance, R., Ader, M. and Silva, L.C. (2010) Noble gas and carbon isotopic signatures of Cape Verde oceanic carbonatites: implications for carbon provenance. Earth and Planetary Science Letters, 291, 70-83.

McGuire, A.V., Francis, C.A. and Dyar, M.D. (1992) Mineral standards for electron microprobe analysis of oxygen. American Mineralogist, 77, 1087-1091.

Mitchell, J.G., Le Bas, M.J., Zielonka, J. and Furnes, H. (1983) On dating the magmatism of Maio, Cape Verde Islands. Earth and Planetary Science Letters, 64, 61-76.

Mourão, C., Mata, J., Doucelance, R., Madeira, J., Brum Da Silviera, A., Silva, L.C. and Moreira, M. (2010) Quaternary extrusive calciocarbonatite volcanism on Brava Island (Cape Verde): a nephelinite-carbonatite immiscibility product. Journal of African Earth Sciences, 56, 59-64.

Mourtada, S., LeBas, M.J. and Pin, C. (1997) Pétrogenèse des magnésio-carbonatites du complexe de Tamazert (Haut Atlas marocain). Comptes Rendues Academie des Sciences Paris, 325, 559-564.

Nasraoui, M. and Bilal, E. (2000) Pyrochlores from the Lueshe carbonatite complex (Democratic Republic of Congo): a geochemical record of different alteration stages. Journal of Asian Earth Sciences, 18, 237-251.

Otto, J.W. and Wyllie, P.J. (1993) Relationships between silicate melts and carbonate-precipitating melts in $\mathrm{CaO}-\mathrm{MgO}-\mathrm{SiO}_{2}-\mathrm{CO}_{2}-\mathrm{H}_{2} \mathrm{O}$ at 2 kbar. Mineralogy and Petrology, 48, 343-365.

Pilet, S., Hernandez, J., Sylvester, P. and Poujol, M.
(2005) The metasomatic alternative for ocean island basalt chemical heterogeneity. Earth and Planetary Science Letters, 236, 148-166.

Plesner, S., Holm, P.M. and Wilson, J.R. (2002) ${ }^{40} \mathrm{Ar}-{ }^{39} \mathrm{Ar}$ geochronology of Santo Antão, Cape Verde Islands. Journal of Volcanology and Geothermal Research, 120, 103-121.

Prestvik, T., Torske, T., Sundvoll, B. and Karlsson, H. (1999) Petrology of early Tertiary nephelinites off mid-Norway. Additional evidence for an enriched endmember of the ancestral Iceland plume. Lithos, 46, 317-330.

Seifert, W., Kämpf, H. and Wasternack, J. (2000) Compositional variation in apatite, phlogopite and other accessory minerals of the ultramafic Delitzsch complex, Germany: implication for cooling history of carbonatites. Lithos, 53, 81-100.

Serralheiro, A. (1976) A geologia da Ilha de Santiago (Cabo Verde). Boletim de Museu e Labóratorio de Mineralogia e Geologia Faculdade de Ciência, 14, $1-157$.

Silva, L., Le Bas, M.J. and Robertson, A.H.F. (1981) An oceanic carbonatite volcano on Santiago, Cape Verde Islands. Nature, 294, 644-645.

Stoppa, F. and Liu, Y. (1995) Chemical composition and petrogenetic implications of apatites from some ultra-alkaline Italian rocks. European Journal of Mineralogy, 7, 391-402.

Subbotin, V.V and Subbotina, G.F. (2000) Pyrochlore group minerals from phoscorites and carbonatites of the Kola Peninsula. Vestnik MGTU, 3, 273-284, [in Russian].

Sun, S.S. and McDonough, W.F. (1989) Chemical and isotopic systematics of oceanic basalts: implications for mantle composition and processes. Pp. 313-345 in: Magmatism in the Ocean Basins. (A.D. Saunders, and M.J. Norry, editors). Geological Society of London, Special Publication, 42. The Geological Society, London.

Ulrych, J., Nižňansky, D., Pertlik, F., Giester, G., Ertl, A. and Brandstätter, F. (2006) Clinopyroxene from an alkali pyroxenite xenolith, LouènáOberwiesenthal Volcanic Centre, Bohemian Massif: crystal chemistry and structure. Geological Quarterly, 50, 257-264.

Veksler, I.V., Petibon, C., Jenner, G.A., Dorfman, A.M. and Dingwell, D.B. (1998) Trace element partitioning in immiscible silicate-carbonate liquid systems: an initial experimental study using a centrifuge autoclave. Journal of Petrology, 39, 2095-2104.

Wallace, M.E. and Green, D.H. (1988) An experimental determination of primary carbonatite magma composition. Nature, 335, 343-346.

Walter, A.V., Nahon, D., Flicoteaux, R., Girard, J.P. and Melfi, A. (1995) Behaviour of major and trace elements and fractionation of REE under tropical 
weathering of a typical apatite-rich carbonatite from Brazil. Earth and Planetary Science Letters, 136, 591-602.

Weaver, B.L. (1991) The origin of ocean island basalt end-member compositions: trace element and isotopic constraints. Earth and Planetary Science Letters, 104, 381-397.

Williams, C.T., Wall, F., Woolley, A.R. and Phillipo, S. (1997) Compositional variation in pyrochlore from the Bingo carbonatite, Zaïre, Journal of African Earth Sciences, 25, 137-145.

Wones, D.R. and Eugster, H.P. (1965) Stability of biotite: experiment, theory and application. American Mineralogist, 50, 1228-1272.

Woolley, A.R. and Kempe, D.R.C. (1989) Carbonatites: nomenclature, average chemical compositions and element distribution. Pp. 1-14 in: Carbonatites: Genesis and Evolution (K. Bell, editor). Unwin Hyman, London.

Wyllie, P.J. and Lee, W.J. (1998) Model system controls on conditions for formation of magnesiocarbonatite and calciocarbonatite magmas from the mantle. Journal of Petrology, 39, 1885-1893.

Zaitsev, A. and Polezhaeva, L. (1994) Dolomite-calcite textures in early carbonatites of the Kovdor ore deposit, Kola peninsula, Russia: their genesis and application of calcite-dolomite geothermometry. Contributions to Mineralogy and Petrology, 115, 339-344. 
